Universidade de São Paulo

Instituto de Física de São Carlos

Cesar Augustus Uliana Lima

\title{
Aspectos termodinâmicos da gravitação semi-clássica
}

São Carlos 

Cesar Augustus Uliana Lima

\title{
Aspectos termodinâmicos da gravitação semi-clássica
}

Dissertação apresentada ao Programa de Pós-graduação em Física do Instituto de Física de São Carlos da Universidade de São Paulo para obtenção do título de Mestre em Ciências.

Área de concentração: Física Básica.

Orientador: Prof. Dr. Daniel Augusto Turolla Vanzella.

\author{
Versão Corrigida \\ (A versão original encontra-se na unidade que aloja o Programa.)
}

São Carlos 
AUTORIZO A REPRODUÇÃO E DIVULGAÇÃO TOTAL OU PARCIAL DESTE TRABALHO, POR QUALQUEER MEIO CONVENCIONAL OU ELETRÔNICO PARA FINS DE ESTUDO E PESQUISA, DESDE QUE CITADA A FONTE.

Ficha catalográfica elaborada pelo Serviço de Biblioteca e Informação do IFSC com os dados fornecidos pelo(a) autor(a)

Uliana Lima, Cesar Augustus

Aspectos Termodinâmicos da Gravitação Semiclássica / Cesar Augustus Uliana Lima; orientador Daniel Augusto Turolla Vanzella - versão corrigida -- São Carlos, 2013

$128 \mathrm{p}$.

Dissertação (Mestrado - Programa de Pós-Graduação em Física Básica) -- Instituto de Física de São Carlos, Universidade de São Paulo, 2013.

1. Termodinâmica. 2. Relatividade geral. 3. Buracos negros. I. Turolla Vanzella, Daniel Augusto, orient. II. Título. 




\section{Dedicatória}

Dedico essa dissertação e todo trabalho nela envolvido a memória de minha mãe, Zélia Uliana, na esperança de que tivesse ela visto os rumos que minha vida tomou teria aprovado o que me sucedeu. 



\section{Agradecimentos}

Agradeço a meu orientador, Professor Daniel Vanzella, pela confiança depositada bem como pela paciência nas discussões que tivemos, onde eu eventualmente muito lutei para compreender pontos deveras triviais. Dizer que ele me ajudou além de qualquer expectativa razoável de um orientador seria uma grave atenuação dos fatos. Aos companheiros de sala, William e Landulfo, por inúmeras vezes terem se disposto a discutir os problemas com os quais eu lidava e muito me ensinaram. Essa dissertação é em parte de vocês. Aos meus amigos de longa data, Daniel, Igor, Renan, Vitor, Jessica, Camila Machado, Bianca, Hugo e Camila Belotti; por todas as coisas que fazem da amizade um conceito que desafia a compreensão e ao mesmo tempo algo imprescindível a vida. Também a meu pai, Julio, e minha irmã, Flavia, sem os quais não haveria uma palavra aqui escrita.

Este trabalho foi suportado por uma bolsa do Conselho Nacional de Desenvolvimento Científico e Tecnológico (CNPq). 

(...) O futuro do mundo será, ao final das contas, decidido pelo o que vocês, os jovens, desejarem. Se a juventude escolher a beleza, haverá mais beleza; se optar pela utilidade, haverá mais coisas úteis. Portanto a decisão de cada indivíduo é importante, não apenas para ele próprio, mas para toda a sociedade.

A mãe de Walter, amigo de um jovem Heisenberg O binómio de Newton é tão belo quanto a Vénus de Milo. O que há é pouca gente para dar por isso. 



\section{Resumo}

ULIANA, C. A. Aspectos termodinâmicos da gravitação semi-clássica. 2013. 128 p. Dissertação (Mestrado em Ciências) - Instituto de Física de São Carlos, Universidade de São Paulo, São Carlos, 2013.

Essa dissertação consiste de uma revisão dos resultados clássicos sobre a termodinâmica de buracos negros bem como de uma análise crítica das extensões recentes da relação entre a termodinâmica e a dinâmica gravitacional e suas implicações.

Palavras-chave: Termodinâmica. Gravitação. Buraco negro. 



\begin{abstract}
ULIANA, C. A. Thermodynamical aspects of semi-classical gravity. 2013. 128 p. Dissertação (Mestrado em Ciências) - Instituto de Física de São Carlos, Universidade de São Paulo, São Carlos, 2013.

This dissertation consists of a revision of the classical results concerning the thermodynamics of black holes as well as a critical analysis of the recent extensions of the relationship between thermodynamics and the gravitational dynamics and its implications.
\end{abstract}

Keywords: Thermodynamics. Gravitation. Black hole. 



\section{Sumário}

1 Introdução 17

2 Sobre a Óptica do Espaço-Tempo 21

2.1 Escalares Ópticos . . . . . . . . . . . . . . . . . . 22

2.2 O Dual de Hodge . . . . . . . . . . . . . . . . . 25

2.3 A Complexidade da Curvatura . . . . . . . . . . . . . 29

2.4 A Classificação de Petrov . . . . . . . . . . . . . . . . . . 33

2.5 O Formalismo de Newman-Penrose . . . . . . . . . . . . . . . 38

2.6 Um Teorema sobre Tipos Algébricos . . . . . . . . . . . . . . 44

3 Estrelas Escuras 49

3.1 Colapso Gravitacional e Singularidades . . . . . . . . . . . . . 49

3.2 Os Buracos Negros . . . . . . . . . . . . . . . . 52 52

3.3 O Estado Final . . . . . . . . . . . . . . . . . . 56

4 O Espaço-Tempo de Kerr 61

4.1 O Giro Inicial $\ldots \ldots \ldots \ldots \ldots$

4.2 Processos Dinâmicos em Buracos Negros . . . . . . . . . . . . . 64

4.3 Unicidade e Calvície . . . . . . . . . . . . . . . . . 68

5 Termodinâmica de Buracos Negros

5.1 Bekenstein e Hawking . . . . . . . . . . . . . . . . . 71

5.2 As Leis da Mecânica de Buracos Negros . . . . . . . . . . . . . . . 74

5.3 O Postulado de Nernst . . . . . . . . . . . . . . . 78 
5.4 A Transição de Davies . . . . . . . . . . . . . . . . . . 82

6 Entropia e Carga de Noether 85

6.1 O Potencial Simplético e a Carga de Noether . . . . . . . . . . . 86

6.2 A Primeira Lei da Dinâmica dos Buracos Negros . . . . . . . . . . . 88

6.3 O caso da Relatividade Geral . . . . . . . . . . . . . . . . . . 91

7 Dinâmica Gravitacional e Termodinâmica 93

7.1 O Horizonte Local de Rindler . . . . . . . . . . . . . . . . . . . 94

7.2 As Equações de Einstein sob Nova Perspectiva . . . . . . . . . . . . 95

7.3 Da Termodinâmica para a Gravitação(?) . . . . . . . . . . . . 98

8 Conclusões e Perspectivas 101

Referências

Apêndice A Formas de Cartan e Tetradas Nulas 111

A.1 O Método do Referencial Móvel . . . . . . . . . . . . . . . . . . 111

A.2 O Referencial Nulo . . . . . . . . . . . . . . . . . . . . . . 112

Apêndice B Tópicos de Estrutura Global 119

B.1 Causalidade . . . . . . . . . . . . . . . . . . . . 119

B.2 Asymtopia . . . . . . . . . . . . . . . . . 121

Apêndice C Congruências Geodésicas 123

C.1 Campos de Jacobi . . . . . . . . . . . . . . . . . . . . . . . . 123

C.2 Formação de Singularidades . . . . . . . . . . . . . . . . . 126 


\section{Capítulo 1}

\section{Introdução}

1 T $\begin{aligned} & \text { o começo do século XX a Relatividade Geral surgiu como uma teoria de gravi- } \\ & \text { tação que incorporava as lições sobre causalidade e localidade da Relatividade }\end{aligned}$ Restrita. Junto com a explicação de fenômenos como a precessão do perihélio de Mercúrio e as lentes gravitacionais, a sua maior contribuição conceitual foi à cosmologia. Ainda assim, durante a primeira metade do referido século a Relatividade Geral foi pouco estudada, seja pela emergência da mecânica quântica, pela falta de experimentos ou pela necessidade dos físicos terem que lidar com técnicas pouco usuais de geometria diferencial, a saber o cálculo tensorial.

Tal situação mudou a partir da década de 1960 e o ímpeto para tal é de certo modo surpreendente. Não foi a escassez de problemas da mecânica quântica (havia o esforço para formular a teoria quântica de campos), nem o surgimento de experimentos e nem a familiaridade com técnicas matemáticas outrora desconhecidas. Pelo contrário, foi a introdução de novos métodos matemáticos, de topologia e álgebra em especial, que lançaram luz sobre a relatividade. Como produto dessa nova direção descobrimos que os buracos negros obedecem leis análogas às da termodinâmica. A subsequente descoberta da radiação Hawking demonstrou que a temperatura formal associada aos buracos negros via a analogia entre a termodinâmica e a Relatividade Geral correspondia de fato a uma temperatura física e assim os estabeleceu como sistemas termodinâmicos de direito. 
A presente dissertação pretende discutir a emergência da termodinâmica no contexto da Relatividade Geral e apreciar os recentes resultados que relacionam as equações da dinâmica gravitacional com a primeira lei da termodinâmica, dentro do âmbito da gravitação clássica.

No capítulo 2 introduzimos um formalismo para curvas nulas que é especialmente adaptado aos problemas de buracos negros dado que estes se caracterizam pelo seu isolamento causal de observadores arbitrariamente distantes. No capítulo 3 discutimos o colapso gravitacional e formação de buracos negros, bem como o teorema da área de Hawking e o estado final do colapso. O capítulo 4 é dedicado à métrica de Kerr e a como extrair energia de um buraco negro.

De posse dos resultados anteriores, e com o auxílio de um efeito semi-clássico, podemos finalmente no capítulo 5 formular a termodinâmica dos buracos negros. Além disso, aproveitamos para discutir certos aspectos menos comentados da termodinâmica de tais objetos. Com isso encerramos a discussão dos resultados clássicos referentes a termodinâmica em contextos gravitacionais.

O capítulo 6 concerne duas discussões ainda não feitas sobre a termodinâmica, a saber o papel das equações da dinâmica e o conceito de densidade de entropia, motivados por novos métodos matemáticos para a primeira lei da dinâmica dos buracos negros. Extrapolando essas lições investigamos uma não-tão-recente proposta de inverter a lógica e deduzir as equações de Einstein tendo postulado a termodinâmica. Por fim no capítulo 8 oferecemos nossas conclusões e perspectivas.

Por motivos de espaço e tempo muitos tópicos que mereceriam seus próprios capítulos foram relegados aos apêndices, os dignos de apêndices relegados às referências, e alguns que deveriam ser citados foram omitidos. Por outro lado é possível que o leitor acredite que alguns capítulos deveriam ser menores e que certas seções poderiam ser omitidas sem prejuízo. A justificativa para certa prolixidade e a inclusão de certos tópicos é devida a opinião do autor que o caminho mais longo eventualmente é o mais recompensador do ponto de vista do entendimento e que alguns assuntos já foram suficientemente negligenciados na literatura em geral a despeito de sua importância. 
Para o leitor que se sentir agravado e discordar das escolhas feitas oferecemos desde já nossas desculpas.

Por fim, é necessário tecer comentários rápidos sobre a notação. Empregamos a métrica de assinatura $(-,+,+,+)$ e a representamos ora por $\mathbf{g}$ ora por $(\mid)$ quando denotamos o produto interno induzido por ela. Em geral usamos a notação livre de índices para tensores por acreditar que a maior parte das discussões é mais transparente desse modo. Nessa notação vetores são representados por letras romanas em negrito como k, formas diferenciais por letras gregas, eventualmente em negrito ou não de acordo com o contexto, como $\omega$ ou $\boldsymbol{\beta}$ e tensores gerais por letras romanas sansserif como R. Subscritos e sobrescritos, ou seja índices, gregos não fazem referência a notação usual para tensores mas indicam que aquele objeto pertence a um conjunto, enumerável ou não, e o índice grego representa um elemento geral no conjunto indexador. Eventualmente pode ser o caso que essa representação não seja a mais conveniente; nessas ocasiões utilizaremos a notação de índices abstratos devida a Penrose [1]. 


\section{Capítulo 2}

\section{Sobre a Óptica do Espaço-Tempo}

1 Pós o fim da Segunda Guerra Mundial o matemático russo Alexei Zinovievich Einstein que mostrava que estes poderiam ser classificados em 3 classes distintas de acordo com propriedades algébricas do seu tensor de curvatura de Weyl [2]. Tal classificação, chamada de Petrov em sua homenagem, se mostrou imensamente relevante para o estudo de ondas gravitacionais.

A razão por detrás da utilidade da classificação de Petrov para o estudo de ondas gravitacionais se deve ao fato que o tipo de espaço-tempo está intimamente relacionado com propriedades de suas geodésicas nulas, tornando esta uma ferramenta própria para a investigação da propagação da luz. A relação da propagação da luz em um espaço-tempo qualquer com suas propridades causais será o fator de interesse para este trabalho. Aproveitaremos para investigar como essa classificação algébrica da curvatura se relaciona com a estrutura complexa natural de um espaço-tempo quadridimensional. Por fim, enunciamos um teorema sobre a geometria das geodésicas nulas.

*Uma variedade, riemanniana ou lorentziana, é dita um espaço de Einstein caso Ric $=\lambda \mathbf{g}$ onde $\lambda$ é uma constante. 


\subsection{Escalares Ópticos}

Os escalares ópticos são funções que descrevem as propriedades geométricas de geodésicas nulas [3]. Para estudá-los convém utilizar uma tetrada nula real.

Definição 2.1.1 Uma tetrada nula real é uma base do espaço tangente $\{\mathbf{k}, \boldsymbol{\ell}, \mathbf{x}, \mathbf{y}\}$ tal que $(\mathbf{k} \mid \boldsymbol{\ell})=-1,(\mathbf{x} \mid \mathbf{x})=(\mathbf{y} \mid \mathbf{y})=+1$ e todos os outros produtos internos são nulos.

Seja agora $\mu$ uma geodésica nula parte de uma congruência geodésica nula de campo tangente $\mathbf{k}$. Dado que $\left(\nabla_{\mathbf{X}} \mathbf{k} \mid \mathbf{k}\right)=1 / 2 \nabla_{\mathbf{X}}(\mathbf{k} \mid \mathbf{k})=0$ para qualquer vetor $\mathbf{X}$ temos que $\nabla_{\mathbf{x}} \mathbf{k} \in \mathbf{k}^{\perp}=\operatorname{span}\{\mathbf{k}, \mathbf{x}, \mathbf{y}\}$. Em especial, caso o campo tangente seja parametrizado afinamente então $\nabla_{\mathbf{k}} \mathbf{k}=0 \Rightarrow \nabla_{\mathbf{X}+\alpha \mathbf{k}} \mathbf{k}=\nabla_{\mathbf{X}} \mathbf{k}$ onde $\alpha$ é uma constante real. Note que isso implica que a conexão de Levi-Civita induz uma relação de equivalência no espaço ortogonal a k. O conjunto das classes de equivalência assim obtidas é o espaço quociente $\mathbf{k}^{\perp} / \mathbf{k}$ onde $\mathbf{X} \equiv \mathbf{Y} \Leftrightarrow \mathbf{X}=\mathbf{Y}+\alpha \mathbf{k}$. Se $\mathbf{X}, \mathbf{Y} \in \mathbf{k}^{\perp} / \mathbf{k}$ então a métrica induz um produto escalar no espaço quociente, dado que $(\mathbf{X}+\alpha \mathbf{k} \mid \mathbf{Y}+\beta \mathbf{k})=(\mathbf{X} \mid \mathbf{Y})$. Usando a tetrada nula real como base do espaço tangente fica claro que o espaço quociente é bidimensional com produto interno induzido positivo definido.

Definição 2.1.2 Seja $\mathfrak{B}: \mathbf{k}^{\perp} / \mathbf{k} \rightarrow \mathbf{k}^{\perp} / \mathbf{k}$ o operador linear dado por $\mathbf{x} \mapsto \nabla_{\mathbf{x}} \mathbf{k}$, então $\mathfrak{B}$ é dito ser o operador forma. Se $\mathbf{x}, \mathbf{y}$ formam uma base ortonormal de $\mathbf{k}^{\perp} / \mathbf{k}$ e $\mathfrak{B}^{x}{ }_{y}=\left(\nabla_{\mathbf{x}} \mathbf{k} \mid \mathbf{y}\right)$ então a matriz correspondente ao operador forma nessa base é

$$
[\mathfrak{B}]=\left[\begin{array}{cc}
\mathfrak{B}_{x}^{x} & \mathfrak{B}^{x} y \\
\mathfrak{B}_{x}^{y} & \mathfrak{B}^{y} y
\end{array}\right] .
$$

Definição 2.1.3 Seja $\mathbf{k}$ um campo vetorial tangente a uma congruência geodésica nula e $\mathfrak{B} o$ operador forma definido em $\mathbf{k}^{\perp} / \mathbf{k}$. Então os escalares ópticos de $\mathbf{k}$ são

1. expansão $\theta=\operatorname{trace}(\mathfrak{B})$

2. vorticidade $\omega, 4 \omega^{2}=\operatorname{det}\left(\mathfrak{B}-\mathfrak{B}^{\top}\right)$

3. cisalhamento $\sigma, 4|\sigma|^{2}=-\operatorname{det}\left(\mathfrak{B}+\mathfrak{B}^{\top}-\theta \mathbb{1}\right)$ 
Note que a expansão é um invariante com relação a base de $\mathfrak{B}$, mas a vorticidade não está definida com relação a um sinal e o cisalhamento com relação a uma fase. Estes podem ser escolhidos arbitrariamente. Com relação a base $\{\mathbf{x}, \mathbf{y}\}$ de $\mathbf{k}^{\perp} / \mathbf{k}$ os escalares podem ser escritos $\theta=\left(\mathfrak{B}^{x}{ }_{x}+\mathfrak{B}^{y} y\right), \omega=\frac{1}{2}\left(\mathfrak{B}^{x}{ }_{y}-\mathfrak{B}^{y}{ }_{x}\right) e \sigma=\frac{1}{2}\left(\mathfrak{B}^{y}{ }_{y}-\mathfrak{B}^{x}{ }_{x}\right)+\frac{i}{2}\left(\mathfrak{B}^{x}{ }_{y}+\mathfrak{B}^{y} x\right)$.

Proposição 2.1.4 Seja $\mathbf{k}$ o campo tangente a uma congruência geodésica nula, então $\mathbf{k}^{\perp} e^{2}$ integrável se, e somente se, $\omega=0$.

Demonstração Se a vorticidade é nula então $\mathfrak{B}^{x}{ }_{y}=\mathfrak{B}^{y}{ }_{x}=\left(\nabla_{\mathbf{x}} \mathbf{k} \mid \mathbf{y}\right)=\left(\nabla_{\mathbf{y}} \mathbf{k} \mid \mathbf{x}\right)$ o que dado $\nabla_{\mathbf{x}}(\mathbf{k} \mid \mathbf{y})=0$ implica $\left(\nabla_{\mathbf{x}} \mathbf{y} \mid \mathbf{k}\right)=\left(\nabla_{\mathbf{y}} \mathbf{x} \mid \mathbf{k}\right) \Rightarrow(\mathbf{k} \mid[\mathbf{x}, \mathbf{y}])=0$. Dado que $(\mathbf{k} \mid[\mathbf{k}, \mathbf{x}])=$ $(\mathbf{k} \mid[\mathbf{k}, \mathbf{y}])=0$ e $\mathbf{k}^{\perp}=\operatorname{span}\{\mathbf{k}, \mathbf{x}, \mathbf{y}\}$ então pelo teorema de Frobenius [4] vorticidade nula é equivalente a integrabilidade de $\mathbf{k}^{\perp}$.

Proposição 2.1.5 Seja k o campo tangente a uma congruência geodésica nula, e a tetrada real nula é transportada paralelamente ao longo de $\mathbf{k}$, então

$$
\begin{aligned}
& \mathbf{k}[\theta]=-\frac{1}{2} \theta^{2}+2 \omega^{2}-2|\sigma|^{2}-\operatorname{Ric}(\mathbf{k}, \mathbf{k}), \\
& \mathbf{k}[\omega]=-\theta \omega, \\
& \mathbf{k}[\sigma]=-\theta \sigma+\frac{1}{2}\left(\mathbf{R}_{\mathbf{k x k} \mathbf{x}}-\mathbf{R}_{\mathbf{k y k y}}\right)-i \mathbf{R}_{\mathbf{k x k y}},
\end{aligned}
$$

onde R denota o tensor de Riemann e Ric o tensor de Ricci. O fato de a equação para o cisalhamento ser complexa ao passo que as para a expansão e vorticidade são reais sugere que podemos combinar as últimas em uma única equação complexa. Para isso definimos $\rho=-\theta / 2+i \omega$ que facilmente verifica-se leva às equações de Sach $\mathrm{s}^{*}$

$$
\begin{aligned}
\mathbf{k}[\rho] & =\rho^{2}+|\sigma|^{2}+\frac{1}{2} \operatorname{Ric}(\mathbf{k}, \mathbf{k}) \\
\mathbf{k}[\sigma] & =(\rho+\bar{\rho}) \sigma+\frac{1}{2}\left(\mathbf{R}_{\mathbf{k x k x}}-\mathbf{R}_{\mathbf{k y k y}}\right)-i \mathbf{R}_{\mathbf{k x k y}} .
\end{aligned}
$$

A demonstração dessa proposição será fornecida na seção 2.5. Para melhor compreender as equações de Sachs considere um pequeno objeto opaco separado de uma tela por ${ }^{*}$ Rainer Kurt Sachs (1932-) 
uma distância finita embora arbitrariamente pequena que intercepte uma congruência geodésica nula de modo a formar uma sombra na tela. Se $d v$ for a distância infinitesimal entre o objeto e a tela então a sombra aumentará de uma fração $\theta d v$, será rotacionada de $\omega d v$ e sofrerá uma cisalhamento $|\sigma| d v[3]$. Note que a fase de $\sigma$ representa o ângulo entre a direção principal de cisalhamento e os vetores da base espacial.

Para que fique claro considere um feixe de luz que inicialmente é composto de raios paralelos. É trivial que isso implica em $\rho=\sigma=0$. Então das equações de Sachs vemos imediatamente que, em primeira ordem, o tensor de Ricci representa o poder dióptrico de uma lente ao passo que certas componentes do tensor de Riemman (que é fácil de ver são puramente curvaturas de Weyl por simetria) o astigmatismo [1]. Assim, em geral, no que tange raios de luz, podemos considerar um espaço-tempo curvo como um conjunto de lentes em cada ponto e em cada direção cujo efeito geral é a convergência (ou divergência) bem como o foco astigmático dos raios de luz.

Os efeitos quando da situação geral $\rho, \sigma \neq 0$ são de mesmo modo bem simples. A equação para a vorticidade implica que expansão positiva tende a diminuir a rotação como de se esperar por conservação de momento angular. Além disso, fica claro que se a vorticidade é nula em um ponto da congruência ela assim permanece ao longo da geodésica que passa por aquele ponto. A equação para o cisalhamento diz que a expansão tende a reduzir o cisalhamento; a contribuição da curvatura será precisada quando discutirmos as direções principais nulas. Já a equação da expansão reflete que tanto a própria expansão como o cisalhamento diminuem a taxa de expansão (pois aumentam o momento de inércia) ao passo que rotação o aumenta atuando como uma "força centrífuga". A contribuição do termo de curvatura de Ricci é mais claro à luz das equações de Einstein, a saber dada a condição de energia forte temos que $\operatorname{Ric}(\mathbf{k}, \mathbf{k}) \geq 0$ e portanto a gravitação clássica sempre tem por efeito a convergência dos raios de luz. 


\subsection{O Dual de Hodge}

Na seção anterior vimos como ao estudarmos as propriedades geométricas (e físicas) de geodésicas nulas a introdução de números complexos simplificou a discussão. A priori tal fato poderia ser meramente incidental. Vamos agora argumentar que o espaçotempo possui uma estrutura complexa natural e que esta se relaciona com as curvas nulas.

Seja $V$ um espaço vetorial de dimensão finita sobre o corpo dos reais e $\Lambda^{2}(V)$ o espaço dos bivetores associado a $V$, isto é, o espaço das combinações de produtos exteriores $\mathbf{v} \wedge \mathbf{w}$ onde $\mathbf{v}, \mathbf{w} \in V$ e $\wedge$ é o produto exterior. Naturalmente o espaço dos bivetores é um espaço vetorial, mas queremos introduzir um produto escalar nele. Para isso vamos usar a propriedade do mapeamento universal [5]: dado uma função bilinear antissimétrica $A: V \times V \rightarrow \mathbb{R}$ existe uma função linear $\ell: \Lambda^{2} \rightarrow \mathbb{R}$ tal que $A(\mathbf{x}, \mathbf{y})=\ell(\mathbf{x} \wedge \mathbf{y})$ para $\forall \mathbf{x}, \mathbf{y} \in V$.

Considere o mapeamento de $V^{4}$ em $\mathbb{R}$ dado por

$$
(\mathbf{v}, \mathbf{w}, \mathbf{x}, \mathbf{y}) \mapsto\left|\begin{array}{cc}
(\mathbf{v} \mid \mathbf{x}) & (\mathbf{v} \mid \mathbf{y}) \\
(\mathbf{w} \mid \mathbf{x}) & (\mathbf{w} \mid \mathbf{y})
\end{array}\right|,
$$

que é multilinear e antissimétrico com respeito ao primeiro e ao segundo par de vetores. Através dele podemos definir o produto escalar em $\Lambda^{2},\langle$,$\rangle , por$

$$
\langle\mathbf{v} \wedge \mathbf{w}, \mathbf{x} \wedge \mathbf{y}\rangle=\left|\begin{array}{cc}
(\mathbf{v} \mid \mathbf{x}) & (\mathbf{v} \mid \mathbf{y}) \\
(\mathbf{w} \mid \mathbf{x}) & (\mathbf{w} \mid \mathbf{y})
\end{array}\right|
$$

estendido por linearidade em todo $\Lambda^{2}$, de modo que é fácil verificar que $\langle\rangle:, \Lambda^{2} \times \Lambda^{2} \rightarrow$ $\mathbb{R}$ é bilinear, simétrica e não-degenerada, isto é sendo $\alpha, \beta \in \Lambda^{2}$ temos que $\langle\alpha, \beta\rangle=0$ para qualquer $\alpha$ implica que $\beta=0$.

Definição 2.2.1 Se $\left\{\mathbf{E}_{\mu}\right\}$ é uma base ortonormal do espaço vetorial $V$ de dimensão $n$ a base canônica de $\Lambda^{2}(V)$ é o conjunto $\left\{\mathbf{E}_{\mu} \wedge \mathbf{E}_{v}\right\}$ com $0 \leq \mu<v<n$. Note que a base canônica assim definida é ortonormal no produto escalar $\langle$,$\rangle .$

Agora que discutimos a estrutura geral dos espaços de bivetores vamos considerar 
o caso particular quando o espaço $V$ é um espaço de Lorentz.

Definição 2.2.2 Seja $\left\{\mathbf{E}_{\mu}\right\}$ uma base ortonormal orientada positivamente, ou seja $\mathbf{E}_{0} \wedge \mathbf{E}_{1} \wedge$ $\mathbf{E}_{2} \wedge \mathbf{E}_{3}=+\omega$ para um espaço de Lorentz $V$ de dimensão $n=4$. Então a base de Hodge é uma base de $\Lambda^{2}(V)$ dada por $\left\{\mathbf{E}_{0} \wedge \mathbf{E}_{1}, \mathbf{E}_{0} \wedge \mathbf{E}_{2}, \mathbf{E}_{0} \wedge \mathbf{E}_{3}, \mathbf{E}_{2} \wedge \mathbf{E}_{3}, \mathbf{E}_{3} \wedge \mathbf{E}_{1}, \mathbf{E}_{1} \wedge \mathbf{E}_{2}\right\}$. Denotando $\epsilon_{\mu v} \equiv \mathbf{E}_{\mu} \wedge \mathbf{E}_{v}$ temos que $\epsilon_{0 v}$ são vetores tipo tempo, $\left\langle\epsilon_{0 \mu}, \epsilon_{0 \mu}\right\rangle=-1$, e $\epsilon_{\mu v}$ com $\mu, v \neq 0$ são tipo espaço, $\left\langle\epsilon_{\mu v}, \epsilon_{\mu v}\right\rangle=+1$.

Note que desse modo segue que $\Lambda^{2}$ é um espaço vetorial de dimensão 6 com assinatura $(-,-,-,+,+,+)$. É justamente a simetria da assinatura que será útil na descrição de geodésicas nulas. Vamos agora investigar como essa simetria introduz uma estrutura complexa.

Lema 2.2.3 Seja $₫$ uma orientação de um espaço de Lorentz V. Então existe um único operador linear $*: \Lambda^{2}(V) \rightarrow \Lambda^{2}(V)$, chamado o dual de Hodge, tal que a identidade de Hodge $\xi \wedge * \eta=$ $-\langle\xi, \eta\rangle @$ vale para quaisquer $\xi, \eta \in \Lambda^{2}$.

Demonstração 1. Unicidade: Suponha que $* \mathrm{e} \star$ sejam dois operadores dual de Hodge distintos. Então

$$
\xi \wedge * \eta=-\langle\xi, \eta\rangle \oplus=\xi \wedge \star \eta \forall \xi, \eta \in \Lambda^{2} .
$$

Tomemos $\eta$ fixo, o que implica $\xi \wedge(* \eta-\star \eta)=0$ para todo $\xi$. Fazendo sucessivamente $\xi=\epsilon_{\mu v}$ para todos elementos da base de $\Lambda^{2}$ resulta em $* \eta-\star \eta=0$. Sendo o operador dual de Hodge linear prova-se a unicidade.

2. Existência: Para mostrar a existência basta enunciar a atuação de * sobre uma base e verificar explicitamente que tal indicação é consistente com as propriedades enunciadas. Se $\left\{\mathbf{E}_{0}, \mathbf{E}_{1}, \mathbf{E}_{2}, \mathbf{E}_{3}\right\}$ é uma base ortonormal orientada positivamente de $V$ tome $*$ definido por

$$
\begin{array}{ll}
*\left(E_{0} \wedge E_{1}\right)=E_{2} \wedge E_{3} & *\left(E_{2} \wedge E_{3}\right)=-E_{0} \wedge E_{1} \\
*\left(E_{0} \wedge E_{2}\right)=E_{3} \wedge E_{1} & *\left(E_{3} \wedge E_{1}\right)=-E_{0} \wedge E_{2}, \\
*\left(E_{0} \wedge E_{3}\right)=E_{1} \wedge E_{2} & *\left(E_{1} \wedge E_{2}\right)=-E_{0} \wedge E_{3} .
\end{array}
$$

\footnotetext{
*Aqui $\omega$ denota um dos vetores unitários de $\Lambda^{4}(V)$ que é isomorfo aos reais. Chamamos $\omega$ de uma orientação para $V$.
} 
Podemos computar facilmente

$$
\mathbf{E}_{\mu} \wedge \mathbf{E}_{v} \wedge *\left(\mathbf{E}_{\lambda} \wedge \mathbf{E}_{\zeta}\right)=-\left\langle\mathbf{E}_{\mu} \wedge \mathbf{E}_{v}, \mathbf{E}_{\lambda} \wedge \mathbf{E}_{\zeta}\right\rangle \omega
$$

para todos os elementos da base. Dado que o operador existe para a base segue da linearidade sua existência para $\Lambda^{2} . \square$

Lema 2.2.4 O operador dual de Hodge * tem as seguintes propriedades:

1. Se $\left\{\mathbf{E}_{0}, \mathbf{E}_{1}, \mathbf{E}_{2}, \mathbf{E}_{3}\right\}$ é uma base ortonormal orientada positivamente de $V$ então sua ação é aquela dada na demonstração do lema 2.2.3.

2. $*^{2}=-\mathrm{id}$, onde id é o operador identidade em $\Lambda^{2}$;

3. *é auto-adjunto, i. e., $\langle * \xi, \eta\rangle=\langle\xi, * \eta\rangle$ para quaisquer $\xi, \eta \in \Lambda^{2}$.

A demonstração da primeira propriedade segue do lema 2.2.3. A segunda propriedade é fácil de verificar dada a atuação na base de $\Lambda^{2}$. A terceira propriedade segue trivialmente uma vez que se nota que o produto exterior $\wedge$ é comutativo para bivetores.

Em função da segunda asserção do lema 2.2.4 vemos que no caso de um espaço vetorial de Lorentz quadridimensional o operador dual de Hodge é uma estrutura complexa no espaço dos bivetores! Mais explicitamente podemos complexificar o espaço $\Lambda^{2}$ definindo a multiplicação por um escalar complexo $a+i b$ por:

$$
(a+i b) \xi=a \xi+b(* \xi), \forall a, b \in \mathbb{R} .
$$

O espaço dos bivetores munido de multiplicação escalar por complexos se torna então tridimensional, sendo uma base possível $\left\{E_{0} \wedge E_{1}, E_{0} \wedge E_{2}, E_{0} \wedge E_{3}\right\}$. Note que a multiplicação pela unidade imaginária fornece os outros vetores da base real. Graças a esse fato podemos denotar essa base resumidamente por $\left\{\varepsilon_{\alpha}\right\}$ onde o $\alpha$ é um índice duplo referente a um dos três primeiros elementos da base de Hodge. Os três elementos posteriores nessa notação são escritos $\left\{\varepsilon_{\alpha^{\prime}}\right\}$ de modo que a ação do dual de Hodge é $* \varepsilon_{\alpha}=\varepsilon_{\alpha^{\prime}} \mathrm{e} * \varepsilon_{\alpha^{\prime}}=-\varepsilon_{\alpha}$. Tanto a base sem linha quanto com linha podem ser usadas para descrever a base de $\Lambda^{2}$ complexificado. Mas para que a complexificação seja completa é importante definir um novo produto escalar sobre o corpo dos complexos. 
Definição 2.2.5 Seja $\boldsymbol{F}(\xi, \eta)=\langle\xi, \eta\rangle-i\langle\xi, * \eta\rangle, \forall \xi, \eta \in \Lambda^{2}$.

Proposição 2.2.6 F é um produto escalar complexo no espaço vetorial $\Lambda^{2}$ complexificado que denotamos $\Lambda_{c x}^{2}$

Para provar que $\boldsymbol{F}$ é um produto escalar complexo precisamos mostrar que ele é sesquilinear e não-degenerado. Como a demonstração dessas propriedades segue diretamente da sua definição através do produto escalar real $\langle$,$\rangle vamos omitir a prova. O leitor$ que não tenha tanta fé no autor pode ver a demonstração em [5].

Lema 2.2.7 Um bivetor $\delta \in \Lambda^{2}(V)$ é nulo com relação $a\langle$,$\rangle e decomponível se, e somente se,$ pudermos expressá-lo por $\mathbf{k} \wedge \mathbf{x}$, onde $\mathbf{k} \in V$ é nulo e $\mathbf{x}$ é tipo espaço e $\mathbf{x} \perp \mathbf{k}$. O vetor $\mathbf{k}$ é único a menos de multiplicação por um escalar de modo que $\delta$ define de modo invariante uma reta nula $N(\delta)=\mathbb{R} \mathbf{k} e m V$.

Demonstração Se o bivetor tem a forma do enunciado é evidente que ele é nulo e decomponível. Agora suponha $\delta=\mathbf{y} \wedge \mathbf{z}$ um bivetor nulo e decomponível. Dado que ele é decomponível então podemos associar a ele uma área orientada dada pelo plano $\Pi=\operatorname{span}\{\mathbf{y}, \mathbf{z}\}$. Por outro lado sendo nulo então $\left.\mathbf{g}\right|_{\Pi}$ é degenerado o que implica que existe um vetor nulo $\mathbf{k}$ único [5] a menos de multiplicação por escalar que pertence ao plano $\Pi$. A reta nula é então $N(\delta)=\Pi \cap \Upsilon$ onde $\Upsilon$ é o cone de luz de $V$.

Note que se $z$ é um número complexo qualquer e $\delta$ um bivetor nulo decomponível então $z \delta$ também é um vetor nulo decomponível que determina a mesma reta nula $N(\delta)$. Portanto os bivetores que determinam $N(\delta)$ são elementos não-zero de uma reta complexa (de caráter tipo-luz) em $\Lambda_{c x}^{2}$.

Proposição 2.2.8 O dual de Hodge em $\Lambda^{2}(V)$ tem as seguintes propriedades:

1. * reverte o caráter causal, o que quer dizer que leva bivetores tipo tempo em tipo espaço e vice-versa, mas leva bivetores nulos em nulos;

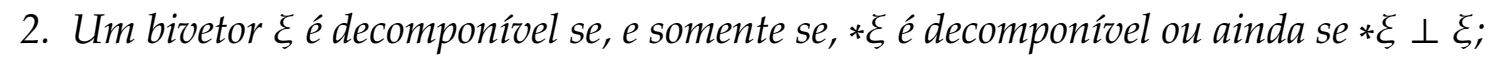

*Um bivetor é dito decomponível se for possível escrevê-lo como produto exterior de dois vetores de $V$. Em especial um bivetor $\xi$ é decomponível se, e somente se, $\xi \wedge \xi=0$. 
3. Se $\delta=\mathbf{k} \wedge \mathbf{x}$ é um bivetor nulo decomponível então $* \delta=\mathbf{k} \wedge \mathbf{y}$, onde $\mathbf{y}$ é um vetor tipo espaço ortogonal a $\mathbf{x}$. Informalmente o dual de Hodge roda o plano definido por $\delta$ de $\pi / 2$ ao redor da reta $\mathbb{R} \mathbf{k}$. Note que os vetores citados são uma base para o espaço ortogonal a $\mathbf{k}$ citado na seção anterior.

Demonstração 1. Dado que $*$ é auto-adjunto e $*^{2}=-i d,\langle * \xi, * \xi\rangle=\langle\xi, * * \xi\rangle=-\langle\xi, \xi\rangle$.

2. Para que um bivetor seja decomponível basta que $\xi \wedge \xi=0$. Que $* \xi \perp \xi$ é fácil de ver por

$$
\langle\xi, * \xi\rangle=0 \Leftrightarrow \xi \wedge * * \xi=0 \Leftrightarrow \xi \wedge \xi=0
$$

Pela simetria do produto escalar se $\xi$ é decomponível $\langle * \xi, \xi\rangle=0 \Rightarrow * \xi \wedge * \xi=0$ e portanto $* \xi$ é decomponível. A volta segue em linhas idênticas facilmente.

3. Dado que $\mathbf{x}$ é tipo espaço então $\mathbf{x}^{\perp}$ é um espaço de Lorentz tridimensional. Vamos escolher uma base para o espaço ortogonal $\left\{\mathbf{E}_{0}, \mathbf{E}_{1}, \mathbf{E}_{2}\right\}$ tal que $\mathbf{k}=c\left(\mathbf{E}_{0}+\mathbf{E}_{1}\right)$, e definimos $\mathbf{E}_{3}=\mathbf{x} / d$, com $d=|\mathbf{x}|$, como o quarto elemento da base de $V$. Dado a linearidade do produto exterior temos

$$
\begin{aligned}
*(\mathbf{k} \wedge \mathbf{x}) & =* c d\left(\mathbf{E}_{0} \wedge \mathbf{E}_{3}+\mathbf{E}_{1} \wedge \mathbf{E}_{3}\right) \\
& =c d\left(\mathbf{E}_{1} \wedge \mathbf{E}_{2}+\mathbf{E}_{0} \wedge \mathbf{E}_{2}\right) \\
& =c\left(\mathbf{E}_{0}+\mathbf{E}_{1}\right) \wedge d \mathbf{E}_{2}=\mathbf{k} \wedge \mathbf{y}
\end{aligned}
$$

onde evidentemente $\mathbf{y}=d \mathbf{E}_{2}$ que tem a propriedade de ser ortogonal ao vetor nulo e ao vetor tipo espaço que formam o bivetor $\delta . \square$

\subsection{A Complexidade da Curvatura}

A classificação de Petrov depende apenas da estrutura algébrica da curvatura no espaço tangente de cada ponto $T_{p}$. Assim vamos ver uma descrição para a curvatura que se aproveite da estrutura do dual de Hodge apenas no caso de uma curvatura definida em um espaço vetorial $V$ de dimensão $n$. O caso típico quando se considera um campo 
tensorial segue diretamente. Ainda mais desse modo ficará evidente que a classificação de Petrov é puramente local.

Definição 2.3.1 Seja $\mathrm{F}$ um tensor de tipo $(0,4) \mathrm{em} V$, ou seja uma função multilinear $\mathrm{F}$ : $V \times V \times V \times V \rightarrow \mathbb{R}$. Então:

1. Se $\mathrm{F}(\mathbf{v}, \mathbf{w}, \mathbf{x}, \mathbf{y})$ é antissimétrico em $\mathbf{v}, \mathbf{w}$ e $\mathbf{x}, \mathbf{y}$ então $\mathrm{F}$ é dito bioblíquo;

2. Se $\mathrm{F}(\mathbf{v}, \mathbf{x}, \mathbf{y}, \mathbf{z})+\mathrm{F}(\mathbf{v}, \mathbf{y}, \mathbf{z}, \mathbf{x})+\mathrm{F}(\mathbf{v}, \mathbf{z}, \mathbf{x}, \mathbf{y})=0$ para quaisquer $\mathbf{v}, \mathbf{x}, \mathbf{y}, \mathbf{z} \in V$ então $o$ tensor é dito ciclicamente simétrico;

3. Se Fé bioblíquo e ciclicamente simétrico então é uma curvatura em $V$.

Corolário 2.3.2 Se F é uma curvatura em $V$ então $\mathrm{F}$ é par-simétrico, isto é $\mathrm{F}(\mathbf{v}, \mathbf{w}, \mathbf{x}, \mathbf{y})=$ $\mathrm{F}(\mathbf{x}, \mathbf{y}, \mathbf{v}, \mathbf{w})$ para todos $\mathbf{v}, \mathbf{w}, \mathbf{x}, \mathbf{y}$ em $V$.

Corolário 2.3.3 Se F é uma cruvatura em $V$ então possui, a menos de um sinal, uma única contração não-nula. Para fixar o sinal definimos a contração por $(\mathbb{E} F)(\mathbf{v}, \mathbf{w})=$ $\sum_{\mu \nu} \mathbf{g}^{\mu \nu} \mathrm{F}\left(\mathbf{E}_{\mu}, \mathbf{v}, \mathbf{E}_{v}, \mathbf{w}\right)$, onde $\mathbf{E}_{\mu}$ são os vetores de uma base arbitrária e $\mathbf{g}^{\mu v}$ a matriz inversa da forma bilinear que faz o produto escalar em $V$. Por motivos puramente psicológicos chamamos a contração $\mathfrak{C F} \equiv$ Ric de curvatura de Ricci de $\mathrm{F}$.

Queremos encontrar uma decomposição natural para a curvatura F. Para isso vamos encontrar uma forma de construir curvaturas.

Definição 2.3.4 Sejam $A$ e $B$ formas bilineares simétricas em $V$. Definamos $(A \wedge B): V^{4} \rightarrow \mathbb{R}$ por

$$
(A \wedge B)(\mathbf{v}, \mathbf{w}, \mathbf{x}, \mathbf{y})=\left|\begin{array}{ll}
A(\mathbf{v}, \mathbf{x}) & A(\mathbf{v}, \mathbf{y}) \\
B(\mathbf{w}, \mathbf{x}) & B(\mathbf{w}, \mathbf{y})
\end{array}\right|
$$

para todos os vetores $\mathbf{v}, \mathbf{w}, \mathbf{x}, \mathbf{y} \in V$. É fácil verificar que $A \wedge B$ é ciclicamente simétrica, porém não necessariamente bioblíqua. No entanto $(A+B) \wedge(A+B)=A \wedge A+A \wedge B+B \wedge A+B \wedge B$ oé. Assim dadas duas formas bilineares simétricas em $V$, Ae $B$, temos a curvatura associada como $A \wedge B+B \wedge A$, já que $A \wedge A$ é uma curvatura também (bastante trivial). 
Sendo $V$ um espaço vetorial de dimensão $n$ e $F$ uma curvatura nele podemos nos perguntar quais são as formas bilineares simétricas inerentes a essa estrutura. Duas muito imediatas são a métrica $\mathbf{g}$ e a curvatura de Ricci Ric. Com elas podemos fazer as seguintes curvaturas: $\mathbf{g} \wedge \mathbf{g}$ e $\mathbf{g} \wedge$ Ric $+\operatorname{Ric} \wedge \mathbf{g}$.

Lema 2.3.5 1. $\mathfrak{C}(\mathbf{g} \wedge \mathbf{g})=(n-1) \mathbf{g}$;

2. $\mathfrak{S}(\mathbf{g} \wedge \operatorname{Ric}+\operatorname{Ric} \wedge \mathbf{g})=(n-2) \operatorname{Ric}+\operatorname{Rg}$, onde Ré o escalar de curvatura, isto é o traço do tensor de Ricci.

A demonstração desse lema segue da computação explícita dos traços. Com isso podemos agora definir a curvatura de Weyl.

Definição 2.3.6 Seja $\mathrm{R}$ uma curvatura em um espaço vetorial $V$ de dimensão $n \geq 3$ munido de um produto escalar. Então o tensor de curvatura conforme de Weyl $\mathrm{C} e$ é definido por

$$
\mathrm{R}=\mathrm{C}+\frac{1}{n-2}(\mathrm{~g} \wedge \mathrm{Ric}+\operatorname{Ric} \wedge \mathrm{g})-\frac{R}{(n-1)(n-2)}(\mathrm{g} \wedge \mathrm{g}) .
$$

Corolário 2.3.7 O tensor de curvatura conforme de Weyl é uma curvatura de traço nulo. Como consequência temos que $\mathrm{C}=0$ para $n=3$.

Lembre agora que segundo a propriedade do mapeamento universal dado uma função bilinear antissimétrica $A: V \times V \rightarrow \mathbb{R}$ existe uma função linear $\ell: \Lambda^{2} \rightarrow \mathbb{R}$ tal que $A(\mathbf{x}, \mathbf{y})=\ell(\mathbf{x} \wedge \mathbf{y})$ para $\forall \mathbf{x}, \mathbf{y} \in V$. Com duas aplicações dessa propriedade podemos mostrar a seguinte proposição.

Proposição 2.3.8 Seja $\mathrm{F}$ uma curvatura em $V$, então existe uma forma bilinear em $\Lambda^{2}(V)$ que denotamos $\mathrm{F}$ também, definida por $\mathrm{F}(\mathbf{v} \wedge \mathbf{w}, \mathbf{x} \wedge \mathbf{y})=\mathrm{F}(\mathbf{v}, \mathbf{w}, \mathbf{x}, \mathbf{y})$ para todos os vetores $\mathbf{v}, \mathbf{w}, \mathbf{x}, \mathbf{y} \in V$ e estendida por linearidade a todo $\Lambda^{2}$.

Vamos olhar o caso de espaços de Einstein, em especial os vácuos Einstein, isto é curvaturas tais que Ric $=0$. Para isso vamos abusar da linguagem e dizer que temos uma curvatura Einstein quando o tensor de Ricci associado a ela for proporcional à métrica. 
1. Fé Einstein se, e somente se, relativa a uma base de Hodge de $\Lambda^{2}(V)$ a matriz da forma bilinear $\mathrm{F}: \Lambda^{2} \times \Lambda^{2} \rightarrow \mathbb{R}$ tem a forma

$$
[\mathrm{F}]=\left[\begin{array}{cc}
A & B \\
B & -A
\end{array}\right]
$$

onde $A$ e $B$ são matrizes $3 \times 3$ simétricas;

2. A simetria cíclica de $\mathrm{F}$ implica que trace $B=0$, e além disso (다), ou seja a sua curvatura de Ricci, é nula se, e somente se, trace $A=0$.

A demonstração dessa proposição segue diretamente de introduzir uma base para $\Lambda^{2}$ e exprimir o fato que Ric $\propto \mathbf{g}$ junto com as simetrias da curvatura herdadas pela forma bilinear. Em particular denotando $F_{\alpha \beta \mu \nu}=\mathrm{F}\left(\mathbf{E}_{\alpha}, \mathbf{E}_{\beta}, \mathbf{E}_{\mu}, \mathbf{E}_{v}\right)$ é fácil verificar que

$$
A=\left[\begin{array}{lll}
F_{0101} & F_{0102} & F_{0103} \\
F_{0201} & F_{0202} & F_{0203} \\
F_{0301} & F_{0302} & F_{0303}
\end{array}\right], B=\left[\begin{array}{lll}
F_{0123} & F_{0131} & F_{0112} \\
F_{0223} & F_{0231} & F_{0212} \\
F_{0323} & F_{0331} & F_{0312}
\end{array}\right] .
$$

A simetria cíclica fornece diretamente $F_{0123}+F_{0231}+F_{0312}=$ trace $B=0$ e das equações para o tensor de Ricci, que nesse caso é $R \mathbf{g}$, temos trace $A=-R$.

Já olhamos a curvatura do ponto de vista da base de Hodge. Queremos agora apreciar como o operador dual de Hodge se relaciona com a curvatura. Para isso vamos usar o fato que todo espaço vetorial com produto escalar contém uma correspondência natural entre formas bilineares e operadores lineares. Assim a forma de curvatura em $\mathrm{F}$ bilinear em $\Lambda^{2}$ determina univocamente um operador linear $\mathbb{F}: \Lambda^{2} \rightarrow \Lambda^{2}$ definido por

$$
\langle\mathbb{F}(\mathbf{v} \wedge \mathbf{w}), \mathbf{x} \wedge \mathbf{y}\rangle=F(\mathbf{v} \wedge \mathbf{w}, \mathbf{x} \wedge \mathbf{y}), \forall \mathbf{v}, \mathbf{w}, \mathbf{x}, \mathbf{y} \in V,
$$

Proposição 2.3.10 Seja $\mathrm{F}$ uma curvatura em $V$, então ela é Einstein se, e somente se, o operador linear correspondente $\mathbb{F}$ em $\Lambda^{2}$ comuta com o operador dual de Hodge *.

Demonstração Dada uma base para $\Lambda^{2}$ temos do lema 2.3.9 que F é Einstein se, e 
somente se, $[\mathrm{F}]$ puder ser escrita como

$$
\left[\begin{array}{cc}
A & B \\
B & -A
\end{array}\right]
$$

e é fácil de verificar que isso implica que $[\mathbb{F}]$ tem de ser da forma

$$
\left[\begin{array}{cc}
-A & -B \\
B & -A
\end{array}\right] .
$$

Agora note que o dual de Hodge na base de Hodge é descrito pela seguinte matriz

$$
[*]=\left[\begin{array}{cc}
0 & -\mathbb{1} \\
\mathbb{1} & 0
\end{array}\right] .
$$

Resta apenas verificar que $[*, \mathbb{F}]=0$ ocorre se, e somente se o operador linear associado a curvatura tiver a forma indicada, o que segue de álgebra elementar.

Lema 2.3.11 Seja $\mathbb{F}$ o operador linear em $\Lambda^{2}$ associado a uma curvatura $\mathrm{F}$ em $V$. Se a matriz de $\mathbb{F}$ tem a forma

$$
[\mathrm{F}]=\left[\begin{array}{cc}
-A & -B \\
B & -A
\end{array}\right]
$$

relativa a uma base $\left\{\varepsilon_{\alpha}, \varepsilon_{\alpha^{\prime}}\right\}$ então a matriz $[\mathbb{F}]_{c x}$ do operador em $\Lambda_{c x}^{2}$ é dada por $[-A+i B]$ relativa tanto a base $\left\{\varepsilon_{\alpha}\right\}$ quanto $a\left\{\varepsilon_{\alpha^{\prime}}\right\}$.

Proposição 2.3.12 $S e_{c x} \mathbb{F}$ é o operador linear em $\Lambda_{c x}^{2}$ associado a uma curvatura Einstein de traço nulo então trace $c_{c x} \mathbb{F}=0$.

Ambas as demonstrações podem ser encontradas em [5].

\subsection{A Classificação de Petrov}

A relação entre curvaturas Einstein e operadores lineares em $\Lambda_{c x}^{2}$ é especialmente frutífera pois permite uma classificação natural das curvaturas, a saber a classificação de Petrov. Para entender de que modo isso se dá convém relembrar certos resultados básicos da álgebra linear. Dado que operadores lineares em espaços vetorias de dimensão 
finita são representáveis por matrizes podemos classificar tais operadores através da forma canônica de Jordan円

Em resumo considere todas as matrizes $n \times n$ que podem ser levadas umas às outras por transformações de similaridade, isto é mudanças de base. É trivial que as transformações de similaridade são uma relação de equivalência no conjunto de matrizes $n \times n$ e portanto particiona tal conjunto em classes de equivalência. A forma canônica de Jordan é simplesmente uma representação única de cada classe de equivalência, o que significa dizer que essa classficação independe da base escolhida. Uma vantagem dessa classificação é que o espectro de uma matriz também é invariante por transformações de similaridadet

Além disso lembre que com relação ao espectro de uma matriz cada autovalor possui uma multiplicidade algébrica e uma multiplicidade geométrica. A multiplicidade algébrica de um autovalor é a multiplicidade da raiz correspondente do polinômio característico. Já a multiplicidade geométrica de um autovalor é a dimensão do autoespaço correspondente. Note que multiplicidade geométrica é necessariamente menor ou igual à algébrica. Mais ainda, a soma das multiplicidades algébricas é igual a dimensão do espaço vetorial. Dada a desigualdade citada isso implica que um operador é diagonalizável se, e somente se, a soma das multiplicidades geométricas é idêntica a dimensão do espaço vetorial.

Lema 2.4.1 (Classificação de Petrov) Seja $\mathrm{C} \neq 0$ uma curvatura de traço nulo em um espaço vetorial lorentziano de dimensão 4 . Então se $\mathbb{C}$ é o operador linear induzido por $\mathrm{C}$ em $\Lambda_{c x}^{2}$ a curvatura está em uma, e apenas uma, das seguintes categorias:

- Tipo I: $\mathbb{C}$ tem três autovalores distintos;

- Tipo D: $\mathbb{C}$ tem dois autovalores distintos, um deles de multiplicidade geométrica 2;

- Tipo II: $\mathbb{C}$ tem dois autovalores distintos, ambos de multiplicidade geométrica 1;

\footnotetext{
*Marie Ennemond Camille Jordan (1838-1922)

${ }^{+}$A classificação original de Petrov [2] era referente a curvaturas Einstein, o que implicava que o traço do operador não era necessariamente nulo. Assim ele classificou as curvaturas apenas com respeito à forma canônica de Jordan, resultando em 3 tipos distintos. Vamos aqui impor o traço nulo o que restringirá o espectro do operador permitindo separar em mais tipos distintos.
} 
- Tipo N: $\mathbb{C}$ tem um autovalor único, necessariamente 0, de multiplicidade geométrica 2;

- Tipo III: $\mathbb{C}$ tem um autovalor único, necessariamente 0 , de multiplicidade geométrica 1.

As curvaturas de Tipo I são ditas algebricamente gerais e as outras algebricamente especiais. Eventualmente é dito que $\mathrm{C}=0$ éalgebricamente especial de Tipo $\mathrm{O}$. Além disso cabe citar que o Tipo D é eventualmente chamado de Tipo II - II ou Tipo II degenerado e o caso Tipo N pode ser chamado em algumas referências por Tipo IV.

A demonstração desse lema é bastante evidente, basta considerar todas as formas canônicas de Jordan possíveis para uma matriz $3 \times 3$ e impor a condição de traço nulo ao espectro. Convém notar que se a curvatura for Einstein mas não de traço nulo podemos repetir a classificação de Petrov mas não será possível separar os casos I de $D$ e nem II de N.

Apesar da classificação via a forma canônica de Jordan ser muito direta e transparente, se ela tem alguma desvantagem certamente é o fato de que o significado geométrico, e portanto físico, não fica evidente. Para esclarecer isso precisamos de um novo conceito.

Definição 2.4.2 Seja $\delta \in \Lambda_{c x}^{2}$ um bivetor nulo com relação ao produto escalar $\boldsymbol{F} e \mathbb{C}$ o operador linear complexo induzido por uma curvatura de Weyl. Então se o bivetor satisfaz $\boldsymbol{F}(\mathbb{C}(\delta), \delta)=0$ então dizemos que $\delta$ é um bivetor nulo principal de $\mathrm{C}$ e a direção $N(\delta)$ que ele determina em $V$ é chamada direção principal nula gravitacional e todo vetor não zero pertencente a linha um vetor principal nulo gravitacional.

É evidente da definição de direção principal nula que se, em particular, um bivetor nulo é autovetor do operador induzido pela curvatura então ele é um bivetor principal nulo. Com isso em mente vamos definir a multiplicidade da direção principal nula.

Definição 2.4.3 Um vetor principal nulo $\mathbf{k} \in N(\delta)$ de C tem multiplicidade m:

- $m=1$ se $\delta$ não éautovetor de $\mathbb{C}$;

- $m=2$ se $\delta$ é autovetor de $\mathbb{C}$ com autovalor não nulo; 
- $m=3$ se $\mathbb{C}(\delta)=0$ e $\operatorname{dim} \operatorname{Ker}(\mathbb{C})=1$;

- $m=4$ se $\mathbb{C}(\delta)=0$ e $\operatorname{dim} \operatorname{Ker}(\mathbb{C})=2$.

Se $\mathbf{k}$ tem multiplicidade m então é dito um vetor nulo m-principal. Em particular se $m=1$ é dito simples e se $m>1$ repetido.

Essas são as únicas possibilidades para curvatura não nula. Vamos agora ver como as direções principais nulas se relacionam com os tipos de Petrov.

Proposição 2.4.4 Considere uma curvatura $\mathrm{C} \neq 0$ de traço nulo, então as suas direções principais nulas gravitacionais e seu tipo de Petrov se relacionam por:

- Tipo I: se possui 4 direções principais nulas simples;

- Tipo D: se possui duas direções 2-principais nulas;

- Tipo II: se possui uma direção 2-principal e duas direções principais nulas simples;

- Tipo N: se possui uma direção 4-principal nula;

- Tipo III: se possui uma direção 3-principal e uma direção nula simples.

A demonstração dessa proposição é um tanto longa e pouca iluminadora e portanto a omitiremos. O leitor interessado pode encontrá-la em [5] por exemplo. De posse dessa proposição podemos agora ver claramente que as curvaturas algebricamente especiais tem direções nulas privilegiadas, a saber aquelas que determinam autovetores nulos do operador $\mathbb{C}$. No entanto é possível que o que significa serem direções privilegiadas não seja claro geometricamente falando. Seria talvez melhor se tivéssemos uma relação entre as direções principais nulas e a curvatura diretamente.

Proposição 2.4.5 (Critério de Sachs) Seja C uma curvatura de traço nulo e $\mathbf{k}$ um vetor nulo. Então:

1. $\mathbf{k}$ é um vetor nulo principal se, e somente se, $\mathbf{C}(\mathbf{k}, \mathbf{x}, \mathbf{k}, \mathbf{y})=0$ para todos $\mathbf{x}, \mathbf{y} \perp \mathbf{k}$;

2. $\mathbf{k}$ é um vetor nulo m-principal com $m \geq 2$ se, e somente se, $\mathbf{C}(\mathbf{k}, \mathbf{x}, \mathbf{k}, \cdot)=0$ para todo $\mathbf{x} \perp \mathbf{k}$; 
3. $\mathbf{k}$ é um vetor nulo m-principal com $m \geq 3$ se, e somente se, $\mathrm{C}(\mathbf{k}, \mathbf{x}, \cdot, \cdot)=0$ para todo $\mathbf{x} \perp \mathbf{k}$;

4. $\mathbf{k}$ é um vetor nulo 4-principal se, e somente se, $\mathrm{C}(\mathbf{k}, \cdot, \cdot, \cdot)=0$.

O critério de Sachs por fim nos dá a forma clara de compreender a física por detrás da classificação de Petrov. Note que o critério de Sachs afirma que se temos um vetor principal nulo então certas componentes do tensor de Weyl são nulas. Pois bem, é digno de nota que ao olharmos as equações de Sachs (2.1.3) as componente nulas são justamente aquelas que aparecem na equação para o cisalhamento (2.1.3b). Como já discutimos o cisalhamento concerne a deformação dos raios de luz. Segue então que a direção principal nula em um certo ponto do espaço-tempo é aquela para a qual um feixe de luz tangente a direção é focado pela curvatura como uma lente sem astigmatismo.

Resta saber qual a diferença entre os diversos tipos de Petrov do ponto de vista físico. Uma análise detalhada, para a qual oferecemos apenas os resultados gerais, pode ser encontrada em [6]. Em suma, devido às componentes do tensor de Weyl que se anulam dependendo da multiplicidade da direção principal nula podemos verificar [7] que no caso do TIPO $\mathrm{N}$ temos propagação de ondas transversais com vetor de propagação colinear ao vetor nulo principal. Do mesmo modo vemos que o Tipo III contém não só ondas transversais como também ondas longitudinais. Por outro lado um conjunto de partículas dispostas como uma esfera teste em um espaço de Tipo D é deformada em uma elipse onde o semi-eixo maior coincide com a direção principal nula, comportamento característico de partícula sob um campo coulombiano. O caso do Tipo II é mais complexo em que ele contém ondas gravitacionais transversais e longitudinais mais o campo tipo-inverso-quadrado. Por fim o caso algebricamente geral se nota por não haver direção privilegiada para as ondas gravitacionais.

Que seja possível entender a classificação de Petrov segundo a ação da gravidade localmente já seria digno de nota, porém mais interessantemente podemos demonstrar um teorema que estende essa analogia. 
Teorema 2.4.6 (Peeling-Off) Considere um espaço-tempo assintoticamente simples [B.2.1]. Seja $\mu$ uma geodésica nula de um ponto qualquer no espaço-tempo para $\mathscr{I}^{+}$(o infinito futuro nulo) e $\lambda$ um parâmetro afim para esta geodésica, de modo que o limite $\lambda \rightarrow \infty$ corresponde a um ponto no infinito futuro nulo. Então no limite do parâmetro afim tendendo ao infinito o tensor de Weyl é assintoticamente

$$
\mathrm{C}=\frac{\mathrm{N}}{\lambda}+\frac{\mathrm{III}}{\lambda^{2}}+\frac{\mathrm{II}}{\lambda^{3}}+\frac{\mathrm{I}}{\lambda^{4}}+O\left(\lambda^{-5}\right)
$$

onde $\mathrm{N}$ denota uma curvatura de Tipo N, III uma curvatura de Tipo III, II uma curvatura de tipo Tipo II ou Tipo D, e I uma curvatura algebricamente geral. Em função disso o primeiro termo é chamado radiativo, o segundo semi-radiativo [8].

Por fim gostaríamos de terminar esta seção notando que devido ao fato de que o campo eletromagnético é descrito por um tensor antissimétrico podemos definir um operador linear induzido e classificá-lo de acordo com as formas canônicas de Jordan [9]. Em resumo a "classificação de Petrov" para o campo eletromagnético no vácuo leva a dois tipos algébricos distintos, o tipo nulo onde os campos elétricos e magnéticos são perpendiculares e portanto representa ondas eletromagnéticas e o tipo não-nulo que corresponde ao comportamento coulombiano. No caso eletromagnético temos também um teorema análogo a 2.4.6 e vemos que o tipo-nulo decai com $\lambda^{-1}$ e o tipo não-nulo $\operatorname{com} \lambda^{-2}$.

\subsection{O Formalismo de Newman-Penrose}

Como vimos ao longo desse capítulo existe uma relação íntima entre a geometria de geodésicas nulas e uma estrutura complexa natural dos espaços-tempo de dimensão 4. A estrutura complexa está relacionada com as direções principais nulas do tensor de Weyl e como descrito na seção 2.1 a física das geodésicas nulas é evidenciada quando escolhemos uma base para o espaço tangente adaptada às direções nulas. 
O formalismo de Newman円Penrose $\mathrm{f}^{円}$ (NP) é um modo mais simétrico de descrever geodésicas nulas inspirado em técnicas de espinores [10].

Definição 2.5.1 Seja $\{\mathbf{k}, \boldsymbol{\ell}, \mathbf{x}, \mathbf{y}\}$ uma tetrada nula real. Definamos os vetores complexos

$$
\mathbf{m}=\frac{1}{\sqrt{2}}(\mathbf{x}+i \mathbf{y}), \overline{\mathbf{m}}=\frac{1}{\sqrt{2}}(\mathbf{x}-i \mathbf{y}) ;
$$

então $\{\mathbf{k}, \boldsymbol{\ell}, \mathbf{m}, \overline{\mathbf{m}}\}$ é uma tetrada nula complexa. Note que como $\mathbf{x}, \mathbf{y}$ são ortonormais $\mathbf{m}, \overline{\mathbf{m}}$ são vetores nulos, isto é $(\mathbf{m} \mid \mathbf{m})=(\overline{\mathbf{m}} \mid \overline{\mathbf{m}})=0 e(\mathbf{m} \mid \overline{\mathbf{m}})=+1$. Assim a métrica $\mathbf{g}$ assume a seguinte forma

$$
[g]=\left[\begin{array}{cccc}
0 & -1 & 0 & 0 \\
-1 & 0 & 0 & 0 \\
0 & 0 & 0 & +1 \\
0 & 0 & +1 & 0
\end{array}\right],
$$

A conexão de Levi-Civita é representada pelas derivadas nas direções da base por $D=\nabla_{\mathbf{k}}$, $\Delta=\nabla_{\ell}, \delta=\nabla_{\mathrm{m}} e \bar{\delta}=\nabla_{\overline{\mathrm{m}}}$.

O "espírito" do formalismo de Newman-Penrose é em certo sentido o oposto do formalismo tensorial, com o que queremos dizer que ao invés de termos equações compactas utilizando tensores em NP escreve-se a equação para cada componente, resultando em um conjunto de equações para funções escalares. A princípio pode parecer que a panacéia de equações resultantes torna impraticável o uso de tal método, mas veremos que quando estamos interessados em geodésicas nulas as equações se tornam simples e reveladoras. Primeiro vamos estudar os análogos dos símbolos de Christoffel.

Definição 2.5.2 Seja $\{\mathbf{k}, \boldsymbol{\ell}, \mathbf{m}, \overline{\mathbf{m}}\}$ uma tetrada nula complexa, então os coeficientes de rotação de Ricci são dados por 12 funções complexas

${ }^{*}$ Ezra Ted Newman (1929-).

${ }^{\dagger}$ Sir Roger Penrose (1931-). 


$$
\begin{array}{ccc}
\kappa=-(D \mathbf{k} \mid \mathbf{m}) & \nu=(\Delta \boldsymbol{\ell} \mid \overline{\mathbf{m}}) & \varepsilon=\frac{1}{2}[-(D \mathbf{k} \mid \boldsymbol{\ell})+(D \mathbf{m} \mid \overline{\mathbf{m}})] \\
\rho=-(\bar{\delta} \mathbf{k} \mid \mathbf{m}) & \mu=(\delta \boldsymbol{\ell} \mid \overline{\mathbf{m}}) & \gamma=\frac{1}{2}[+(\Delta \boldsymbol{\ell} \mid \mathbf{k})-(\Delta \overline{\mathbf{m}} \mid \mathbf{m})] \\
\sigma=-(\delta \mathbf{k} \mid \mathbf{m}) & \lambda=(\bar{\delta} \boldsymbol{\ell} \mid \overline{\mathbf{m}}) & \beta=\frac{1}{2}[-(\delta \mathbf{k} \mid \boldsymbol{\ell})+(\delta \mathbf{m} \mid \overline{\mathbf{m}})] \\
\tau=-(\Delta \mathbf{k} \mid \mathbf{m}) & \pi=(D \boldsymbol{\ell} \mid \overline{\mathbf{m}}) & \alpha=\frac{1}{2}[+(\bar{\delta} \boldsymbol{\ell} \mid \mathbf{k})-(\bar{\delta} \overline{\mathbf{m}} \mid \mathbf{m})]
\end{array}
$$

que são chamados coeficientes de spin. Note que as 12 funções complexas são equivalentes às 24 componentes independentes dos coeficientes de rotação de Ricci. A distribuição irregular de letras para as funções se deve que mediante a reflexão de tetrada $\mathbf{k} \leftrightarrow \boldsymbol{\ell} e \mathbf{m} \leftrightarrow \overline{\mathbf{m}}$ produz-se a mudança

$$
\kappa \leftrightarrow-v, \rho \leftrightarrow-\mu, \sigma \leftrightarrow-\lambda, \tau \leftrightarrow-\pi, \varepsilon \leftrightarrow-\gamma, \beta \leftrightarrow-\alpha,
$$

Assim poderíamos ter definido apenas 6 funções e a reflexão de tetrada para definir todos os 24 graus de liberdade da forma de conexão.

O motivo para a escolha de algumas letras para os coeficientes de spin se relaciona com o fato que expandindo os vetores complexos da base em suas partes reais e imaginárias é trivial verificar que $\rho$ e $\sigma$ são a divergência complexa e o cisalhamento presentes nas equações de Sachs (2.1.3). Para compreendermos o significado geométrico dos outros coeficientes de spin precisamos expandir os vetores da forma $\nabla_{\mathbf{X}} \mathbf{Y}$ na base de NP, obtendo, por exemplo

Corolário 2.5.3 Uma tetrada complexa nula $\{\mathbf{k}, \boldsymbol{\ell}, \mathbf{m}, \overline{\mathbf{m}}\}$ possui derivadas covariantes

$$
\begin{aligned}
D \mathbf{k} & =(\varepsilon+\bar{\varepsilon}) \mathbf{k}-\bar{\kappa} \mathbf{m}-\kappa \overline{\mathbf{m}} \\
D \boldsymbol{\ell} & =-(\varepsilon+\bar{\varepsilon}) \mathbf{k}+\tau \mathbf{m}+\overline{\tau \mathbf{m}} \\
D \mathbf{m} & =\bar{\pi} \mathbf{k}-\kappa \boldsymbol{\ell}+(\varepsilon-\bar{\varepsilon}) \mathbf{m} \\
\Delta \mathbf{k} & =(\gamma+\bar{\gamma}) \mathbf{k}-\bar{\tau} \mathbf{m}-\tau \overline{\mathbf{m}} \\
\delta \mathbf{m} & =\bar{\lambda} \mathbf{k}-\sigma \boldsymbol{\ell}+(\beta-\bar{\alpha}) \mathbf{m}
\end{aligned}
$$

As outras derivadas podem ser encontradas em A.2.1.

Da equação 2.5.4a podemos ver claramente que $\kappa=0$ implica que a curva nula de tangente $\mathbf{k}$ é pré-geodésica e que se além disso $\varepsilon+\bar{\varepsilon}=0$ então ela é geodésical $\mathrm{A}$ *Aqui reservamos a palavra pré-geodésica em referência a curvas tais que $\nabla_{\mathbf{X}} \mathbf{X} \propto \mathbf{X}$ e geodésica para 
equação (2.5.4d) revela que $\tau$ mede a deformação em $\mathbf{k}$ quando propagado na direção de $\boldsymbol{\ell}$ e (2.5.4e) que $\alpha$ e $\beta$ estão relacionados com as mudanças em $\mathbf{m}$ (e seu complexo conjugado) embora o fato de que nenhum dos dois apareçam sozinhos nas equações das derivadas impeça uma interpretação mais clara do papel de ambos.

Assim temos que $\rho$ é a divergência complexa de $\mathbf{k}, \sigma$ o cisalhamento, $\kappa$ e $\varepsilon$ refletem a geodicidade da curva, $\tau$ a propagação na direção nula complementar. As funções $\mu, \lambda, v, \gamma, \pi$ são equivalentes mediante a reflexão da tetrada, isto é tem as mesmas propriedades mas para a direção $\ell$ e $\alpha, \beta$ se relacionam com as direções do plano espacial ortogonal embora não de forma tão transparente.

Além disso por uma simples inspeção de (2.5.4a), (2.5.4b) e (2.5.4c) nota-se que se $\kappa=\varepsilon=\pi=0$ então a tetrada é transportada paralelamente na direção $\mathbf{k}$. Isso implica que se a parte real de $\varepsilon$ refletia o parâmetro afim da geodésica nula então sua parte imaginária mede a rotação da base da secção espacial ortogonal. Do mesmo modo como notado na proposição 2.1.4 se $\rho-\bar{\rho}=0$ então $\mathbf{k}$ é ortogonal a hiperfícies, em outras palavras o vetor é proporcional a um campo gradiente. Se além disso tivermos $\tau=\bar{\alpha}+\beta$ então ele é igual a um campo gradiente [10].

Frequentemente ao lidarmos com problemas geométricos existem escolhas que simplificam a descrição e por conseguinte a solução do problema. Vimos a ligação dos coeficientes de spin com certas propriedades geométricas das curvas às quais são tangentes. Gostaríamos de saber quanta liberdade temos em mudar os coeficientes escolhendo uma base apropriada para o espaço tangente. Sabendo que as tetradas representam referenciais móveis, vide apêndice $\mathrm{A}$. queremos saber como transformações de Lorentz afetam os coeficientes.

Definição 2.5.4 Seja $\{\mathbf{k}, \boldsymbol{\ell}, \mathbf{m}, \overline{\mathbf{m}}\}$ uma tetrada complexa nula. Existem funções complexas $b, c$ e funções reais $a, \vartheta$ que descrevem as transformações de Lorentz ou rotações da tetrada que se dividem nas seguintes classes:

- Classe I: $\mathbf{k}$ invariante, $\mathbf{m} \rightarrow \mathbf{m}+c \mathbf{k}, \overline{\mathbf{m}} \rightarrow \overline{\mathbf{m}}+\bar{c} \mathbf{k} e \boldsymbol{\ell} \rightarrow \boldsymbol{\ell}+\bar{c} \mathbf{m}+c \overline{\mathbf{m}}+|c|^{2} \mathbf{k}$;

- Classe II: idêntica a Classe I porém com $\mathbf{k}$ e $\boldsymbol{\ell}$ trocados $e b \leftrightarrow c$;

aquelas que além disso tem parâmetro afim, em conformidade com [1, [5]. 
- Classe III: $\mathbf{k} \rightarrow a^{-1} \mathbf{k}, \boldsymbol{\ell} \rightarrow a \boldsymbol{\ell}, \mathbf{m} \rightarrow e^{i \vartheta} \mathbf{m} e \overline{\mathbf{m}} \rightarrow e^{-i \vartheta} \overline{\mathbf{m}}$, essa rotação é particularmente conhecida por spin-boost dado que se $\vartheta=0$ temos os boosts de Lorentz usuais.

A relação completa de como mudam os coeficientes de spin sob rotações gerais se encontra na seção A.2

Por simples inspeção das fórmulas para rotações podemos ver que dada uma tetrada complexa nula qualquer podemos sempre fazer uma rotação de Classe II e escolher $\kappa=0$. Agora podemos fazer uma rotação de Classe III, que não altera $\kappa$, que consiste apenas de um spin $(a=0)$ para escolher $\varepsilon=0$. Por fim usando uma rotação de Classe I, que não altera $\kappa$ ou $\varepsilon$ neste caso, escolhemos $\pi=0$. Assim dada uma geodésica nula genérica sempre podemos encontrar uma tetrada nula complexa em que um dos vetores nulos reais é tangente e com parâmetro afim e tal que a tetrada é transportada paralelamente ao longo da geodésica.

Vamos investigar agora a curvatura do ponto de vista do formalismo de NewmanPenrose.

Definição 2.5.5 Seja $\{\mathbf{k}, \boldsymbol{\ell}, \mathbf{m}, \overline{\mathbf{m}}\}$ uma tetrada complexa nula em um espaço-tempo. Os escalares de Weyl da tetrada são as funções complexas

$$
\begin{gathered}
\Psi_{0}=\mathrm{C}(\mathbf{k}, \mathbf{m}, \mathbf{k}, \mathbf{m}), \Psi_{1}=\mathrm{C}(\mathbf{k}, \boldsymbol{\ell}, \mathbf{k}, \mathbf{m}), \Psi_{2}=\mathrm{C}(\mathbf{k}, \mathbf{m}, \overline{\mathbf{m}}, \boldsymbol{\ell}), \\
\Psi_{3}=\mathrm{C}(\mathbf{k}, \boldsymbol{\ell}, \overline{\mathbf{m}}, \boldsymbol{\ell}), \Psi_{4}=\mathrm{C}(\boldsymbol{\ell}, \overline{\mathbf{m}}, \boldsymbol{\ell}, \overline{\mathbf{m}}) .
\end{gathered}
$$

Tal definição quando comparada ao critério de Sachs [2.4.5] fornece a demonstração diretamente do seguinte lema.

Lema 2.5.6 Se $\mathbf{k}$ é um vetor nulo m-principal então para uma tetrada $\{\mathbf{k}, \boldsymbol{\ell}, \mathbf{m}, \overline{\mathbf{m}}\}$

1. $m \geq 1 \Leftrightarrow \Psi_{0}=0$;

2. $m \geq 2 \Leftrightarrow \Psi_{0}=\Psi_{1}=0$;

3. $m \geq 3 \Leftrightarrow \Psi_{0}=\Psi_{1}=\Psi_{2}=0$;

4. $m=4 \Leftrightarrow \Psi_{0}=\Psi_{1}=\Psi_{2}=\Psi_{3}=0$. 
Um corolário bastante interessante permite um novo critério para a classificação de Petrov.

Corolário 2.5.7 Seja $\{\mathbf{k}, \boldsymbol{\ell}, \mathbf{m}, \overline{\mathbf{m}}\}$ uma tetrada nula complexa arbitrária. Por uma transformação de Classe II podemos sempre anular $\Psi_{0}$ por $\Psi_{0}+4 b \Psi_{1}+6 b^{2} \Psi_{2}+4 b^{3} \Psi_{3}+b^{4} \Psi_{4}=0$. A classificação de Petrov se relaciona com as raizes dessa equação por:

- Tipo I: se as 4 raízes são distintas;

- Tipo II: se possui 3 raízes distintas, uma necessariamente de multiplicidade 2;

- Tipo D: se possui duas raízes distintas, ambas de multiplicidade 2;

- Tipo III: se possui 2 raízes distintas, uma de multiplicidade 3;

- Tipo N: se possui todas as raízes iguais.

A demonstração desse corolário é trivial, basta verificar que se uma raizé múltipla então além de $\Psi_{0}$ ela é raíz de outro dos escalares de Weyl, o que segue de como os outros escalares se transformam por rotações de Classe II. Além disso, para o caso particular do Tipo $D$ temos $\Psi_{0}=\Psi_{1}=\Psi_{3}=\Psi_{4}=0$ e $\Psi_{2} \neq 0$. De acordo com as observações ao final da seção 2.4 se um espaço-tempo é Tipo $D$ em todos os seus pontos então ele é o análogo de uma fonte gravitacional isolada sem nenhuma onda gravitacionalf

Neste ponto esperamos que o leitor esteja convencido de que o formalismo de Newman-Penrose, embora não seja tão sucinto como o tensorial, mais do que compensa o volume de elementos que introduz ao permitir a visualização geométrica de muitas afirmações bem como produzir um critério muito simples para a classificação de Petrov. Ainda assim nos falta ver a forma que assumem as identidades de Ricci nesse formalismo e verificar que estas também contêm um significado geométrico claro.

Proposição 2.5.8 Seja $\{\mathbf{k}, \boldsymbol{\ell}, \mathbf{m}, \overline{\mathbf{m}}\}$ uma tetrada nula complexa e $\Phi_{00}=1 / 2 \operatorname{Ric}(\mathbf{k}, \mathbf{k})$. Então:

$$
\begin{aligned}
& D \rho-\bar{\delta} \kappa=\left(\rho^{2}+|\sigma|^{2}\right)+\rho(\varepsilon+\bar{\varepsilon})-\bar{\kappa} \tau-\kappa(3 \alpha+\bar{\beta}-\pi)+\Phi_{00}, \\
& D \sigma-\delta \kappa=\sigma(\rho+\bar{\rho}+3 \varepsilon-\bar{\varepsilon})-\kappa(\tau-\bar{\pi}+\bar{\alpha}+3 \beta)+\Psi_{0} .
\end{aligned}
$$

*Para ser mais específico deveríamos exigir que o espaço-tempo em questão também seja assintoticamente simples, obviamente. 
A demonstração dessa proposição embora longa é trivial. Uma idéia para a demonstração seria começar com a segunda equação estrutural do lema A.1.4 que fornece as componentes da curvatura em função dos coeficientes de rotação de Ricci. Para o formalismo de Newman-Penrose os coeficientes de rotação de Ricci são os coeficientes de spin, para os quais conhecemos as derivadas e portanto podemos calcular a 2-forma

de curvatura. Basta então verificar que a equação 2.5.5a corresponde a $\Omega^{\mathrm{m}}{ }_{\mathbf{k}}(\mathbf{k}, \overline{\mathbf{m}}) \mathrm{e}$ a equação (2.5.5b) corresponde a $\Omega^{\mathrm{m}}{ }_{\mathbf{k}}(\mathbf{k}, \mathbf{m})$, onde em um abuso de notação utilizamos $\mathbf{X}$ tanto para o vetor quanto para o índice da base como em $\mathbf{E}_{\mathbf{X}}$. As outras identidades de Ricci para a curvatura podem ser encontradas do mesmo modo utilizando todos os vetores da tetrada resultando nas equações A.2.5.

Note que tomando $\kappa=\varepsilon=0$, o que sempre podemos fazer, obtemos as equações de Sachs (2.1.3). Portanto vemos que as equações de Sachs não são nada mais que as equações para o tensor de curvatura expressas utilizando uma tetrada nula complexa específica. As identidades de Ricci no formalismo de Newman-Penrose tem a vantagem então de mostrar como mudam os coeficientes de spin (que já vimos são objetos geométricos) em função da curvatura da variedade, separando inclusive as contribuições dos tensores de Weyl e Ricci. Desse modo sabendo quais escalares de Weyl se anulam em cada tipo de Petrov podemos entender como isso se reflete nas mudanças de congruências nulas. O fato de trocarmos uma única equação que fornece a curvatura em termos dos símbolos de Christoffel [4] por 18 equações complexas parece um preço pequeno a se pagar pelo entendimento do significado das equações.

\subsection{Um Teorema sobre Tipos Algébricos}

A classificação de Petrov fornece um modo claro de classificar curvaturas de traço nulo, e portanto espaços-tempo vácuo, que é ao mesmo tempo clara matematicamente e relevante fisicamente. Vimos que a estrutura algébrica da curvatura se relaciona com direções nulas privilegiadas, as direções principais nulas, nas quais o foco de um feixe de raios de luz incidentes paralelamente no dado ponto se dá sem astigmatismo. 
Para complementar a discussão sobre os tipos algébricos é necessário especificar de que modo as direções principais nulas repetidas são diferentes das direções simples do ponto de vista geométrico. Para isso vamos demonstrar um teorema sobre tipos de Petrov cuja primeira prova é devido a Goldberg冈 e Sachs [11] t e revela que as direções principais repetidas tem uma geometria muito particular e justifica o adjetivo de algebricamente especial.

Essencial para o teorema são as identidades de Bianchi, que no método de Cartan assumem a forma $d \boldsymbol{\Omega}^{\mu}{ }_{v}=\sum_{\sigma} \boldsymbol{\Omega}^{\mu}{ }_{\sigma} \wedge \omega^{\sigma}{ }_{v}+\sum_{\sigma} \omega^{\mu}{ }_{\sigma} \wedge \boldsymbol{\Omega}^{\sigma}{ }_{v}$ [12]. Supondo que o espaçotempo é vácuo $(\mathrm{Ric}=0)$ e tomando os elementos da tetrada nula complexa obtemos as equações A.2.6. De posse das identidades de Bianchi podemos verificar um notável teorema.

Teorema 2.6.1 (Goldberg-Sachs) Em um espaço-tempo com Ric $=0 e \mathrm{C} \neq 0$ uma congruência de curvas nulas é geodésica e livre de cisalhamento se, e somente se, seu campo tangente é uma direção principal nula repetida do tensor de Weyl.

De outro modo, em um espaço-tempo vácuo caso exista uma congruência nula geodésica e de cisalhamento nulo, então o espaço-tempo é algebricamente especial. Note que a afirmação é estritamente local e mostra que congruências geodésicas nulas sem cisalhamento nunca ocorrem no Tipo I.

Demonstração Vamos começar demonstrando que se $\mathbf{k}$ é um vetor m-principal nulo gravitacional então $\mathbf{k}$ égeodésico e com cisalhamento nulo. Essa afirmação segue diretamente das identidades de Bianchi considerando cada multiplicidade. Se $m=2$ para o campo tangente a congruência então o lema 2.5.6 implica que $\Psi_{0}=\Psi_{1}=0$ em um aberto, ou seja não só os escalares como suas derivadas são nulas. Susbtituindo esse dado em A.2.6a) e A.2.6e obtemos $3 \kappa \Psi_{2}=0$ e $3 \sigma \Psi_{2}=0$. Como $\Psi_{2} \neq 0$, caso contrário teríamos $m=3$, isso implica $\kappa=\sigma=0$. Se $m=3$ então $\Psi_{0}=\Psi_{1}=\Psi_{2}=0$. De modo análogo as equações A.2.6b) e (A.2.6f implicam $2 \kappa \Psi_{3}=0$ e $2 \sigma \Psi_{3}=0$ de onde $\kappa=\sigma=0$ pois *Joshua N. Goldberg (1925-).

${ }^{+}$Uma nota histórica, o teorema de Goldberg-Sachs embora tenha sido primeiro demonstrado pelos senhores que o nomeiam teve a sua primeira demonstração impressa em [10]. Cabe notar que o artigo original [11] usa 12 páginas para demonstrar o teorema usando métodos tradicionais ao passo que em [10] através do formalismo NP a demonstração do mesmo teorema ocupa meia página! 
$\Psi_{3} \neq 0$ devido à multiplicidade. Por fim caso $m=4$ temos de (A.2.6c) e (A.2.6g) que $\kappa \Psi_{4}=0$ e $\sigma \Psi_{4}=0$ já que $\Psi_{0}=\Psi_{1}=\Psi_{2}=\Psi_{3}=0$ e como supomos que a curvatura de Weyl não é identicamente nula temos $\kappa=\sigma=0$. Para completar a demonstração basta notar que podemos fazer uma transformação de Classe III que não altera $\kappa=\sigma=0$ para fazer $\varepsilon=0$ e assim mostrar que a congruência é de fato geodésica e com cisalhamento nulo.

Precisamos agora mostrar que se $\mathbf{k} e$ o campo tangente a uma congruência geodésica nula sem cisalhamento então é uma direção principal nula repetida do tensor de Weyl. O lema2.5.6 nos diz que precisamos mostrar que isso implica em $\Psi_{0}=\Psi_{1}=0$. Primeiro vamos utilizar uma rotação de Clase III, que não altera $\kappa=\sigma=0$, para fazer $\varepsilon=0$. Da equação (2.5.5b) temos trivialmente que a nulidade desses escalares implica em $\Psi_{0}=0$, o que leva a uma proposição útil.

Proposição 2.6.2 Se $\{\mathbf{k}, \boldsymbol{\ell}, \mathbf{m}, \overline{\mathbf{m}}\}$ é um campo de tetrada em um aberto onde Ric $=0$ com $\Psi_{0}=0$ e $\kappa=\sigma=\varepsilon=0$ então

$$
\begin{aligned}
D \tau & =\rho(\tau+\bar{\pi})+\Psi_{1} \\
D \beta & =\beta \bar{\rho}+\Psi_{1} \\
\delta \rho & =\rho(\bar{\alpha}+\beta)+\tau(\rho-\bar{\rho})-\Psi_{1} \\
D \Psi_{1} & =4 \rho \Psi_{1} \\
\delta \Psi_{1} & =2(2 \tau+\beta) \Psi_{1}
\end{aligned}
$$

onde as 3 primeiras equações seguem das identidades de Ricci (A.2.5) e as 2 útilmas das identidades de Bianchi A.2.6.

Veja que se $\rho=0$ ao longo do aberto então $\Psi_{1}=0$ segue trivialmente de 2.6.1c). Falta verificar apenas o caso $\rho \neq 0$ em todo o aberto. Quando a expansão complexa não é nula podemos fazer uma transformação de Classe I, que não altera $\kappa=\sigma=\varepsilon=0$, e fazer $\tau=0$ dado que $\tau \rightarrow \tau+c \rho+\bar{c} \sigma+|c|^{2} \kappa$ e só precisamos escolher $c=-\tau / \rho$. Da equação 2.6.1a) segue então que $\Psi_{1}=-\rho \bar{\pi}$. Agora só precisamos mostrar que $\pi=0$.

Para isso note que $\tau=0$ implica que (2.6.1d) e (2.6.1e) podem ser escritas resumidamente $D\left(\ln \Psi_{1}\right)=4 \rho$ e $\delta\left(\ln \Psi_{1}\right)=2 \beta$. Note que o comutador $[D, \delta]$ aplicado a $\ln \Psi_{1}$ 
nos dá

$$
[D, \delta] \ln \Psi_{1}=D(2 \beta)-\delta(4 \rho)=2\left(\beta \bar{\rho}+\Psi_{1}\right)-4\left(\rho(\bar{\alpha}+\beta)-\Psi_{1}\right)=2 \beta \bar{\rho}-4 \rho(\bar{\alpha}+\beta)+6 \Psi_{1}
$$

onde utilizamos (2.6.1b) e (2.6.1c) na segunda igualdade. Porém podemos calcular os comutadores utilizando as expressões A.2.2. Tomando $\kappa=\sigma=\varepsilon=0$ temos que $[D, \delta]=(\bar{\pi}-\bar{\alpha}-\beta) D+\bar{\rho} \delta$ que aplicado a $\ln \Psi_{1}$ resulta

$$
[D, \delta] \ln \Psi_{1}=(\bar{\pi}-\bar{\alpha}-\beta) D\left(\ln \Psi_{1}\right)+\bar{\rho} \delta\left(\ln \Psi_{1}\right)=(\bar{\pi}-\bar{\alpha}-\beta) 4 \rho+\bar{\rho} 2 \beta
$$

onde usamos (2.6.1d) e 2.6.1e) em termos do logaritmo do escalar de Weyl na segunda igualdade. Se compararmos 2.6.2) e 2.6.3) obtemos

$$
2 \beta \bar{\rho}-4 \rho(\bar{\alpha}+\beta)+6 \Psi_{1}=(\bar{\pi}-\bar{\alpha}-\beta) 4 \rho+\bar{\rho} 2 \beta
$$

que implica $6 \Psi_{1}=4 \bar{\pi} \rho$. Comparando com o que obtemos da equação (2.6.1a) temos que $\Psi_{1}=-\rho \bar{\pi}$ e $\Psi_{1}=2 / 3 \rho \bar{\pi}$, e sendo que $\rho \neq 0$ por suposição temos que $\pi=0 \Rightarrow \Psi_{1}=0$ completando a demonstração.

A demonstração desse teorema é interessante por ser um exemplo da metodologia usualmente utilizada no formalismo de Newman-Penrose. Em geral escrevemos explicitamente as equações que envolvem os escalares de interesse, utilizamos transformações de Lorentz para simplificar as expressões, e assim tentamos verificar a veracidade das asserções. Mais ainda o teorema de Goldberg-Sachs mostra que a existência de congruências geodésicas nulas livres de cisalhamento é extremamente extraordinária, em particular nenhuma pode existir, nem mesmo localmente, em espaços algebricamente gerais. Cabe notar que como a classificação de Petrov não é robusta por perturbações, ou seja uma perturbação linear sobre espaços algebricamente especiais leva usualmente ao Tipo I então não esperamos em um espaço-tempo realista que este possua geodésicas nulas de cisalhamento nulo.

A raridade de geodésicas nulas com $\sigma=0$ do ponto de vista físico está de acordo com o papel do cisalhamento no transporte de energia das ondas gravitacionais [8,13] já que uma geodésica com cisalhamento nulo implica na conservação da massa de Bondi. 
Em outras palavras o cisalhamento das geodésicas é diretamente atrelado a geometria, não podendo ser escolhido livremente. 


\section{Capítulo 3}

\section{Estrelas Escuras}

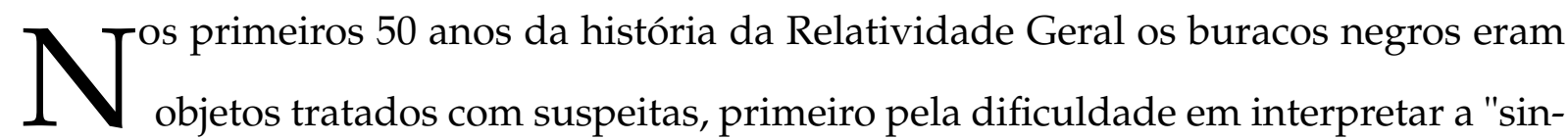

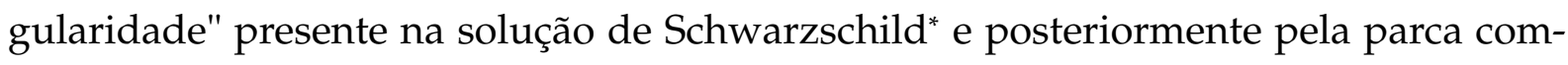
preensão da estrutura e evolução estelar. A introdução de técnicas de geometria e topologia diferencial, principalmente devido a Penrose [1], mudou tal panorama e deu origem à "Era de Ouro da Relatividade Geral". Nesse capítulo vamos discutir os aspectos gerais dos buracos negros, em particular sua relação com a formação de singularidades no espaço-tempo e as características típicas de sua evolução.

\subsection{Colapso Gravitacional e Singularidades}

Com o desenvolvimento das técnicas espectroscópicas no final do século XIX e início do século XX a astronomia se transformou por desenvolver a capacidade de entender a composição das estrelas e foi formada assim a astrofísica moderna. Mas foi com o cálculo do limite de Chandrasekhar $[14]^{\dagger}$ para a massa das anãs brancas, as estrelas cuja pressão interna para contrabalancear a força gravitacional advém do princípio de exclusão de Pauli dos elétrons constituintes, que se deu origem ao conceito de objetos 
compactos em astrofísica. Ainda na década de 1930 Oppenheimer Volkoff [15] e Snyderf [16] mostraram ainda mais que as estrelas de nêutrons, aquelas onde a pressão gravitacional induz o espalhamento beta inverso convertendo prótons dos núcleos em nêutrons, também possuem uma massa limite para a qual a matéria fermiônica degenerada resiste a atração gravitacional.

Em particular, seguindo os trabalhos supracitados, considere a evolução de uma estrela esfericamente simétrica. Dado o teorema de Birkhof [17] temos que o exterior da estrela é representado pela métrica de Schwarzschild. Se a massa da estrela for superior ao limite de Tolman If Oppenheimer-Volkoff, algo entre 2 a 3 massas solares (o valor exato depende da equação de estado para a matéria de nêutrons em regimes de pressão extrema, algo ainda hoje não bem consolidado), então não existe configuração de equilíbrio. Em outras palavras a atração gravitacional é sempre superior a pressão de degenerescência e o colapso gravitacional se torna inevitável. Esse processo então leva a formação de uma singularidade em $r=0$, no sentido de que a curvatura cresce ilimitadamente.

Note no entanto que no caso do colapso esfericamente simétrico efeito análogo está presente mesmo no caso newtoniano, a chamada instabilidade de Jeans [18 É notório que no caso newtoniano a instabilidade de Jeans é prevenida por pequenas perturbações, de modo que o colapso existe apenas na situação idealizada, isto é consiste de um produto das simetrias. Cabe então se perguntar se o mesmo é o caso da Relatividade Geral. O teorema de Penrose [C.2.6] responde isso negativamente. O ponto central consiste da observação que as esferas com $r<2 M$ da solução de Schwarzchild são superfícies aprisionadas fechadas [C.2.5], e que a existência das mesmas implica necessariamente que o espaço-tempo não é geodésicamente completo [B.1.10].

Duas questões surgem de imediato. A primeira é a associação da presença de

\footnotetext{
${ }^{*}$ Julius Robert Oppenheimer (1904-1967)

${ }^{\dagger}$ George Michael Volkoff (1914-2000)

¥Hartland Sweet Snyder (1913-1962)

§George David Birkhoff (1884-1944)

${ }^{\mathbb{I}} \mathrm{O}$ teorema de Birkhoff foi encontrado pelo mesmo em 1923 porém já havia sido derivado independentemente dois anos antes por Jørg Tofte Jebsen (1888-1922).

"Richard Chase Tolman (1881-1948)

${ }^{* *}$ Sir James Hopwood Jeans (1877-1946)
} 
singularidades com a incompletude geodésica. A existência de uma geodésica causal incompleta sinaliza que existem "buracos" no espaço-tempo, com o que queremos dizer que existem geodésicas causais que embora inextensíveis cobrem apenas um alcance finito de parâmetro afim. Poderíamos então tentar introduzir uma fronteira no espaço-tempo para refletir a presença de uma singularidade [17], porém infelizmente todas as sugestões para tal fronteira não são bem sucedidas em caracterizar todos os exemplos de singularidade que temos [4]. Assim tomaremos sempre a incompletude de gedésicas causais por sinônimo da presença de singularidades. A segunda questão é o critério da presença de superfícies aprisionadas fechadas. Elas estão presentes em Schwarzschild e embora um conceito que não assume simetrias não é óbvio sua relação com o colapso gravitacional. Felizmente teoremas por Schoen e Yau e posteriormente por Clarke [19] mostram que sempre aparecem superfícies aprisionadas quando existir uma concentração suficientemente grande de massa em uma região compacta do espaço-tempq

Portanto fica claro que ou a formação de singularidades é inevitável no colapso gravitacional, ou alguma das suposições do teorema de Penrose são violadas, a saber, que aparecem densidades de energia negativas $(\operatorname{Ric}(\mathbf{k}, \mathbf{k})=T(\mathbf{k}, \mathbf{k})<0)$ ou então o espaço-tempo deixa de ser globalmente hiperbólico. Ainda pode ser o caso de que o conceito de espaço-tempo deixe de ser apropriado a partir de certo ponto, sinalizando a emergência de um efeito de gravitação quântica. Note que a hiperbolicidade global implica que o espaço-tempo é fortemente causal [B.1.17], e portato sua violação permite a existência de curvas causais fechadas. Note ainda que o tensor energia-momento de um campo quântico pode violar a condição de energia forte [17]. No intuito de preservar a causalidade somos deixados com duas possibilidades: ou se formam singularidades ou efeitos quânticos devem se fazer presentes.

Para discutir essas possibilidades é necessário precisar o que é um buraco negro.

\footnotetext{
${ }^{*}$ Note no entanto que os teoremas de singularidade não generalizam e sim complementam o resultado de Oppenheimer e Snyder. Isso porque os teoremas de Penrose exigem que a métrica seja suficientemente diferenciável (tipicamente $C^{2}$ ) porém a métrica da estrela sofrendo colapso analisada é contínua porém não diferenciável na superfície. Isso dá ainda mais credibilidade a formação de singularidades durante o colapso.
} 
Intuitivamente desejamos caracterizar um buraco negro como uma região do espaçotempo tal que nenhuma curva causal partindo dessa região se intercepte com um observador assintótico. Uma formalização disso é:

Definição 3.1.1 Seja $(\mathcal{M}, \mathbf{g})$ um espaço-tempo fortemente assintoticamente previsível B.2.6] tal que $\mathcal{M} \not \subset J^{-}\left(\mathscr{I}^{+}\right)$, então o conjunto $B:=\mathcal{M}-J^{-}\left(\mathscr{I}^{+}\right)$é chamado buraco negro. A fronteira de $B, H=M \cap \dot{J}^{-}\left(\mathscr{I}^{+}\right)$é o horizonte de eventos.

Com essa definição em mente percebemos que a existência de um buraco negro permite que tanto a formação de singularidades quanto a presença de efeitos de gravitação quântica possam passar despercebidas por observadores que permanecerem sempre fora do buraco negro. Isso forma o cerne da conjectura da censura cósmica, a saber de que o colapso gravitacional sempre leva a formação de buracos negros e nunca a formação de singularidades nuas. Certamente se conhecem contra-exemplos para tal conjectura, mas todos envolvem casos extremamente específicos, em particular todos presupõe simetrias e não são estváveis por perturbações das mesmas, de modo que existem condições suficientemente razoáveis para descartá-los. Além disso, as situações típicas estudadas motivam tal conjecturaf Assim o status atual da conjectura da censura cósmica é de que aguarda uma formulação rigorosa e dentro desse âmbito ainda mais carece de demonstração, mas parece ser apropriada [20]. Desse modo suporemos tacitamente tal conjectura quando apropriado. Voltaremos a discuti-la no âmbito da termodinâmica na seção 5.3 .

\subsection{Os Buracos Negros}

Agora que estabelecemos que os buracos negros são objetos típicos a serem esperados quando do colapso gravitacional de estrelas suficientemente massivas $\rrbracket^{\top}$ vamos passar a uma discussão mais pormenorizada de suas propriedades. Primeiro vamos precisar

${ }^{*}$ A conjectura da censusa cósmica é um dos mais importantes problemas em aberto da Relatividade Geral. Vamos voltar a essa discussão em um âmbito mais restrito na seção 5.3

${ }^{+}$A palavra massiva não faz parte do léxico da língua portuguesa, mas vamos aqui introduzir tal palavra como um adjetivo que caracteriza a qualidade de possuir massa. 
o que constituem buracos negros em um dado "tempo". As demonstrações aqui não fornecidas podem ser encontradas, por exemplo, em [17].

Definição 3.2.1 Seja $(\mathcal{M}, \mathbf{g})$ um espaço-tempo que possui um buraco negro B. Seja $\Sigma$ uma superfície de Cauchy do espaço-tempo, então $B \cap \sum$ é a região total de buraco negro no tempo $\Sigma$. Cada conjunto conexo $\mathcal{B}$ de $B \cap \Sigma$ é um buraco negro no tempo $\Sigma$.

No caso particular de um espaço-tempo estacionário podemos ainda mostrar que cada buraco negro em um dado tempo tem horizonte de eventos naquele tempo topologicamente $S^{2}[21]$.

Teorema 3.2.2 Seja $(\mathcal{M}, \mathbf{g})$ um espaço-tempo fortemente assintoticamente previsível e $\Sigma_{1}$ e $\Sigma_{2}$ duas superfícies de Cauchy tais que $\Sigma_{2} \subset I^{+}\left(\Sigma_{1}\right)$. Seja $\mathcal{B}_{1}$ um subconjunto conexo não-vazio de $B \cap \Sigma_{1}$, então $J^{+}\left(\mathcal{B}_{1}\right) \cap \Sigma_{2} \neq \emptyset$ e é um conjunto conexo $\mathcal{B}_{2} \subset B \cap \Sigma_{2}$.

Esse teorema garante que cada buraco negro não pode desaparecer conforme ele evolui e mais fortemente deve permanecer conexo o que implica que não pode se bifurcar. As próximas proposições relacionam a existência de buracos negros com superfícies aprisionadas C.2.5.

Proposição 3.2.3 Seja $(\mathcal{M}, \mathbf{g})$ um espaço-tempo fortemente assintoticamente previsível tal que $\operatorname{Ric}(\mathbf{k}, \mathbf{k}) \geq 0$ para qualquer vetor $\mathbf{k}$ nulo. Então se $\mathcal{M}$ contém uma superfície aprisionada fechada $\mathscr{T}$ necessariamente temos que $\mathscr{T} \subset B$, onde $B$ denota um buraco negro no espaçotempo.

Definição 3.2.4 Seja uma superfície tipo espaço bidimensional compacta $\mathscr{T}$ tal que as duas congruências geodésicas nulas ortogonais a $\mathscr{T}$ tem expansão não-positiva em todos os pontos da superfície. Então $\mathscr{T}$ é uma superfície marginalmente aprisionada.

Podemos considerar as superfícies aprisionadas como um caso limite das superfícies marginalmente aprisionadas. De modo análogo a proposição 3.2 .3 temos que todas as superfícies marginalmente aprisionadas são subconjuntos do buraco negro do espaçotempo. 
Definição 3.2.5 Seja $\Sigma$ uma superfície de Cauchy assintoticamente plana. Considere um subconjunto fechado de $\Sigma, C \subset \Sigma \subset \mathcal{M}$ que é uma variedade tridimensional com fronteira $S=\dot{C}$ tal que a expansão das geodésicas nulas ortogonais a $S$ dirigidas ao futuro seja nãopositiva. Então S é uma superfície marginalmente aprisionada exterio情e C uma região aprisionada.

Proposição 3.2.6 Seja $(\mathcal{M}, \mathbf{g})$ um espaço-tempo fortemente assintoticamente previsível tal que $\operatorname{Ric}(\mathbf{k}, \mathbf{k}) \geq 0$ para qualquer vetor $\mathbf{k}$ nulo. Considere uma superfície de Cauchy assintoticamente plana $\Sigma$ e uma região aprisionada $C \subset \Sigma$. Então $C \subset B \cap \Sigma$.

Definição 3.2.7 Seja $(\mathcal{M}, \mathbf{g})$ um espaço-tempo fortemente assintoticamente previsível tal que $\operatorname{Ric}(\mathbf{k}, \mathbf{k}) \geq 0$ para qualquer vetor $\mathbf{k}$ nulo. Considere a família $\left\{C_{\alpha}\right\}$ de todas as regiões aprisionadas, então $\mathscr{T}=\overline{\bigcup_{\alpha} C_{\alpha}}$ é a região aprisionada total e $\mathscr{A}=\dot{\mathscr{T}}$ o horizonte aparente em $\Sigma$.

Teorema 3.2.8 Seja $(\mathcal{M}, \mathbf{g})$ um espaço-tempo fortemente assintoticamente previsível tal que $\operatorname{Ric}(\mathbf{k}, \mathbf{k}) \geq 0$ para qualquer vetor $\mathbf{k}$ nulo. Se existir uma região aprisionada total $\mathscr{T}$ em uma superfície de Cauchy que seja uma variedade com fronteira então o horizonte aparente Á é uma superfície marginalmente aprisionada exterior com expansão $\theta$ nula.

Segue diretamente da proposição 3.2 .6 que o horizonte aparente está sempre contido dentro, ou coincide com, o horizonte de eventos. No entanto para determinar o horizonte de eventos é necessário o conhecimento da evolução completa do buraco negro, ao passo que o horizonte aparente depende apenas de grandezas na superfície de Cauchy. Essa propriedade é particularmente importante quando desejamos estudar a evolução de uma solução numericamente. Se desejamos encontrar o horizonte de eventos tipicamente devemos evoluir o espaço-tempo e no fim traçar as geodésicas nulas para trás no tempo para encontrar o horizonte de eventos. Por outro lado o horizonte aparente pode ser encontrado em qualquer superfície de Cauchy a cada instante da evolução temporal.

\footnotetext{
*Note que não exigimos a compacidade de $C$ no caso das superfícies marginalmente aprisionadas exteriores. Além disso uma superfície marginalmente presa não precisa ser marginalmente presa exterior pois não necessariamente constitui a fronteira de uma variedade tridimensional.
} 
Vamos agora discutir um teorema com relação a evolução do horizonte de eventos, que constitui o resultado central desta seção. Posteriormente no capítulo5ele ocupará igualmente um papel de destaque para estabelecer a relação da mecânica dos buracos negros com a termodinâmica.

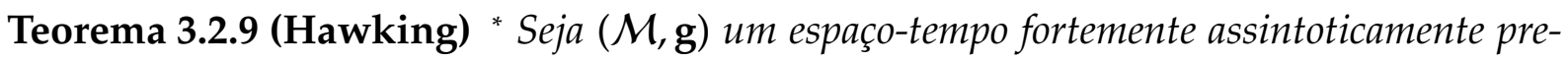
visível tal que $\operatorname{Ric}(\mathbf{k}, \mathbf{k}) \geq 0$ para qualquer vetor $\mathbf{k}$ nulo. Considere duas superfícies de Cauchy $\Sigma_{1}$ e $\Sigma_{2} \operatorname{com} \Sigma_{2} \subset I^{+}\left(\Sigma_{1}\right)$ e denotemos $\mathscr{H}_{\alpha}=H \cap \Sigma_{\alpha}$ onde $H$ é o horizonte de eventos de um buraco negro B. Então a área da superfície $\mathscr{H}_{2}$ é maior ou igual a área de $\mathscr{H}_{1}$.

Demonstração O plano geral da demonstração consiste em provar que a expansão das geodésicas nulas dirigidas ao futuro de $\mathscr{H}_{1}$ que geram o horizonte de eventos é sempre não-negativa. Depois construir um mapa entre os pontos do horizonte de eventos no tempo 1 e os pontos no tempo 2 e o não decréscimo da área decorrerá da nãonegatividade da expansão. Seja $p \in \mathscr{H}_{1}$ e suponha $\theta<0$ nesse ponto. Então podemos deformar $\mathscr{H}_{1}$ continuamente em uma vizinhança do horizonte de eventos obtendo uma nova superfície $\mathscr{H}^{\prime}$ em $\Sigma_{1}$ que também teria geradores convergindo e estaria contida em $J^{-}\left(\mathscr{I}^{+}\right)$. Porém a expansão negativa implica que um ponto $q \in \mathscr{H}^{\prime}$ possui um ponto conjugado em $\dot{J}^{+}\left(\mathscr{H}^{\prime}\right)$ (vide o lema C.1.2 e a proposição C.1.5 e portanto essa geodésica não pode se estenter até $\mathscr{I}^{+}$contrariando a construção. Portanto temos que os geradores de $\dot{J}^{+}\left(\mathscr{H}_{1}\right)$ tem sempre expansão $\theta \geq 0$. Ainda utilizando a propriedade de que o horizonte de eventos $H$ é formado por geodésicas nulas inextensíveis ao futuro, considere dois pontos $p \in \mathscr{H}_{1}$ e $q \in \mathscr{H}_{2}$, ambos pertencentes a uma mesma geodésica nula $\mu$ de $H$. Podemos utilizar tais geodésicas nulas para criar um mapa contínuo entre os pontos de $\mathscr{H}_{1}$ e um subconjunto dos pontos de $\mathscr{H}_{2}$ (note que embora os geradores não possam convergir formando pontos conjugados nada impede de que se formem novos buracos negros) e dado a expansão não-negativa das geodésicas é trivial constatar que a área de tal subconjunto é necessariamente maior ou igual a área do horizonte de eventos no tempo 1 . Portanto a área de $\mathscr{H}_{2}$ nunca é menor que a de $\mathscr{H}_{1}$. 
Uma variação dessa demonstração aliada ao teorema 3.2 .2 permite demonstrar ainda que se existem $N$ regiões de buracos negros conexas em um dado tempo e apenas um buraco negro em uma superfície de Cauchy posterior então necessariamente a área do buraco negro final é maior que a área dos buracos negros originais [17]. Ainda mais cabe notar que o teorema 3.2 .9 apenas garante que a área total nunca decresce, mas poderíamos nos perguntar se dados inúmeros buracos negros em uma dada superfície de Cauchy se um deles não poderia ter uma área decrescente a expensa do crescimento correspondente dos outros de modo a preservar o teorema de Hawking. É notório esse não ser o caso, e que de fato se dados buracos negros em um tempo $\Sigma_{1}$ existir um buraco negro em uma superfície $\Sigma_{2} \subset I^{+}\left(\Sigma_{1}\right)$ com área menor que todos os em $\Sigma_{1}$ esse necessariamente se formou entre os dois tempos [22].

\subsection{O Estado Final}

Considere um buraco negro resultante do colapso gravitacional. Dada a natureza do horizonte de eventos ser uma superfície sem retorno podemos esperar que, pelo menos em algumas situações, dado tempo suficiente o buraco negro deve tender a uma solução estacionária após emitir toda energia disponível na forma de radiação gravitacional. Nesta seção iremos explorar algumas propriedades gerais de buracos negros estacionários, deferindo a discussão das propriedades de uma solução particular para o próximo capítulo. Para tornar a discussão mais compacta utilizaremos a notação de índices abstratos de Penrose.

Para ser preciso estaremos interessados em um espaço-tempo fortemente assintoticamente previsível que contenha um buraco negro e que possua um grupo uniparamétrico de isometrias tal que o campo de Killing [4] correspondente seja tipo-tempo perto de $\mathscr{I}^{+}$. Além disso trataremos especificamente ou de espaços-tempo vácuo ou tais que contenham campos satisfazendo a condição de energia dominante, isto é $T_{a b} \xi^{a} \chi^{b} \geq 0$, para quaisquer vetores tipo-tempo $\xi^{a}, \chi^{b}$, o que equivale a supor que o fluxo de energia conforme visto por um observador é sempre sub-luminal. Vamos começar com uma 
proposição cuja demonstração pode ser encontrada em [17].

Proposição 3.3.1 Seja $(\mathcal{M}, \mathbf{g})$ um espaço-tempo fortemente assintoticamente previsível e estacionário que contenha um buraco negro B. Então os geradores do horizonte de eventos $k^{a}$ dirigidos ao futuro tem expansão $\theta=0$.

Um corolário direto de tal proposição e do teorema 3.2 .8 é que no caso estacionário o horizonte de eventos coincide com o horizonte aparente. Se $\xi^{a}$ for o gerador da isometria temporal ainda assim não necessariamente precisa ser um campo ortogonal a hiperfícies $(\omega=0)$. Um resultado dos teoremas de unicidade, que discutiremos na seção 4.3, no entanto mostra que:

Proposição 3.3.2 Seja $(\mathcal{M}, \mathbf{g})$ um espaço-tempo fortemente assintoticamente previsível e estacionário que contenha um buraco negro B. Se o gerador das isometrias temporais $\xi^{a}$ não for ortogonal a hiperfícies então existe um outro grupo uniparamétrico cíclico ${ }^{\text {de }}$ de isometrias tal que o gerador $\varphi^{a}$ é assintoticamente tipo-espaço além de uma constante $\Omega$ tal que o campo de Killing $\chi^{a}=\xi^{a}+\Omega \varphi^{a}$ é ortogonal a hiperfícies. Em particular o campo $\chi^{a}$ é ortogonal ao horizonte de eventos, de modo que o mesmo também é um horizonte de Killing甲 Caso o intervalo dos parâmetros de $\varphi^{a}$ seja $[0,2 \pi]$ então a constante $\Omega$ é denominada velocidade angular do buraco negro.

Queremos agora explorar a natureza do campo de Killing ortogonal ao horizonte de eventos.

Proposição 3.3.3 Seja $(\mathcal{M}, \mathbf{g})$ um espaço-tempo fortemente assintoticamente previsível e estacionário que contenha um buraco negro B. Considere o campo de Killing ortogonal ao horizonte de eventos $\chi^{a}$, então existe um escalar $\varkappa$ que é constante ao longo das órbitas de $\chi^{a}$.

Demonstração Sendo o horizonte de eventos uma hiperfície nula e $\chi^{a}$ ortogonal a ele é trivial que $\chi^{a} \chi_{a}=0$ no horizonte e em particular $\chi^{a} \chi_{a}=$ cte no mesmo. Portanto $\nabla^{a}\left(\chi^{b} \chi_{b}\right)$ é ortogonal ao horizonte e assim existe uma função $\varkappa$ tal que $\nabla^{a}\left(\chi^{b} \chi_{b}\right)=-2 \varkappa \chi^{a}$. Vamos

\footnotetext{
*Aqui cíclico denota que o parâmetro do grupo pertence a um intervalo fechado $[a, b]$ com os pontos $a \mathrm{e}$ $b$ identificados.

${ }^{\dagger}$ Um horizonte de Killing corresponde a uma hiperfície nula tal que existe um campo de Killing ortogonal a mesma.
} 
tomar a derivada de Lie com relação a $\chi^{a}$ da expressão anterior. O lado esquerdo é trivialmente zero quando lembramos da equação de Killing que a derivada de Lie de um campo vetorial é $£_{\chi} \xi^{a}=[\chi, \xi]^{a}$. Portanto temos

$$
0=£_{\chi} \varkappa\left(-2 \chi^{a}\right)-2 \varkappa £_{\chi} \chi^{a}=£_{\chi} \varkappa\left(-2 \chi^{a}\right) \Rightarrow £_{\chi} \varkappa=0
$$

demonstrando assim o enunciado.

Proposição 3.3.4 Seja $(\mathcal{M}, \mathbf{g})$ um espaço-tempo fortemente assintoticamente previsível e estacionário que contenha um buraco negro B. Considere o campo de Killing ortogonal ao horizonte de eventos $\chi^{a}$ e a função $\varkappa$ constante ao longo de suas órbitas. Se $\chi \neq 0$ então o campo $\chi^{a}$ é pré-geodésico, e geodésico do contrário. Tal função é denominada gravidade superficial.

Demonstração Tomemos a equação que define $\varkappa, \nabla^{a}\left(\chi^{b} \chi_{b}\right)=-2 \varkappa \chi^{a}$. Aplicando a regra de Leibniz no lado esquerdo $2 \chi^{b} \nabla^{a} \chi_{b}=-2 \varkappa \chi^{a}$. Agora utilizando a equação de Killing $\nabla_{a} \chi_{b}+\nabla_{b} \chi_{a}=0$ e a métrica para abaixar índice obtemos $-\chi^{b} \nabla_{b} \chi_{a}=-\varkappa \chi_{a}$ e portanto $\chi^{a}$ satisfaz a equação da pré-geodésica $\chi^{b} \nabla_{b} \chi^{a}=\varkappa \chi^{a}$.

Vamos utilizar o campo $\chi^{a}$ para formar uma tetrada nula complexa $\left\{\chi^{a}, n^{a}, m^{a}, \bar{m}^{a}\right\}$. Nesta base vemos que, em cima do horizonte, temos $\kappa=0$ e $\mathcal{\varkappa}=\varepsilon+\bar{\varepsilon}$. É fácil verificar que podemos fazer uma transformação de Classe III com parâmetros $a=e^{\varkappa v}$ e $\vartheta=0$, onde $D v=1$, para formar uma tetrada nula complexa tal que $\varepsilon+\bar{\varepsilon}=0$. Na base original podemos escrever $\varkappa=-n^{a} \chi^{b} \nabla_{b} \chi_{a}$.

Queremos agora mostrar que $\varkappa$ é constante não só ao longo das órbitas do campo de Killing como também no horizonte inteiro. Mas antes precisamos de um lema que é digno de nota por si mesmo.

Lema 3.3.5 Seja $(\mathcal{M}, \mathbf{g})$ um espaço-tempo fortemente assintoticamente previsível e estacionário que contenha um buraco negro B. Então o horizonte de eventos é uma hiperfície algebricamente especial.

Demonstração Vamos denotar o gerador das geodésicas ortogonais ao horizonte por $k^{a} \equiv e^{-\varkappa v} \chi^{a}$ conforme a rotação de Classe III citada. Portanto temos $\kappa=0$. Agora

$$
k_{[a} \nabla_{b]} k_{c}=k_{a} \nabla_{b} k_{c}-k_{b} \nabla_{a} k_{c}=e^{-\chi v}\left[\left(\chi_{a} \nabla_{v}-\chi_{b} \nabla_{a}\right) e^{-\varkappa v} \chi_{c}\right]
$$




$$
k_{[a} \nabla_{b]} k_{c}=-e^{-2 \varkappa v} \chi_{c}\left[\chi_{[a} \nabla_{b]}(\varkappa v)\right]+e^{-2 \varkappa v}\left[\chi_{[a} \nabla_{b]} \chi_{c}\right]
$$

Dado que $\chi^{a}$ é ortogonal a hiperfícies temos do teorema de Frobenius [4] que $\chi_{[a} \nabla_{b} \chi_{c]}=$ 0 . Abrindo a equação explicitamente e utilizando a equação de Killing é fácil verificar que $2 \chi_{[a} \nabla_{b]} \chi_{c}=-\chi_{c} \nabla_{a} \chi_{b}$. Portanto temos no horizonte

$$
k_{[a} \nabla_{b]} k_{c}=-e^{-2 \varkappa v} \chi_{c}\left[\frac{1}{2} \nabla_{a} \chi_{b}+\chi_{[a} \nabla_{b]}(\varkappa v)\right] .
$$

Contraindo (3.3.4) com $m^{c}$ obtemos $m^{c} k_{a} \nabla_{b} k_{c}=m^{c} k_{b} \nabla_{a} k_{c}$ dado que $m^{c} \chi_{c}=(\mathbf{m} \mid \chi)=0$. Agora contraindo com $m^{b}$ obtemos $m^{c} m^{b} \nabla_{b} k_{c}=(\delta \mathbf{k} \mid \mathbf{m})=-\sigma=0$. Pelo teorema de Goldberg-Sachs 2.6.1 dado que $\kappa=\sigma=0$ então o espaço-tempo é algebricamente especial no horizonte e $k^{a}$ uma direção principal nula repetida.

Note que se na última passagem da demonstração fizermos a contração $\operatorname{com} \bar{m}^{b}$ então $\theta=0$ e demonstramos parte da proposição 3.3.1. Vamos agora passar para o resultado principal desta seção.

Teorema 3.3.6 Seja $(\mathcal{M}, \mathbf{g})$ um espaço-tempo fortemente assintoticamente previsível e estacionário que contenha um buraco negro B e satisfaça a condição de energia dominante. Então a gravidade superficial $\varkappa$ é constante no horizonte de eventos.

Demonstração Já demonstramos esse ser o caso para as órbitas do campo de Killing. Resta então mostrar que $m^{a} \nabla_{a} \varkappa=0$ e tomando o complexo conjugado fica estabelecido o teorema.

$$
\begin{gathered}
m^{c} \nabla_{c} \mathcal{\varkappa}=m^{c} \nabla_{c}\left(-n^{a} \chi^{b} \nabla_{b} \chi_{a}\right), \\
m^{c} \nabla_{c} \mathcal{\varkappa}=-m^{c} n^{a} \chi^{b}\left(\nabla_{c} \nabla_{b} \chi_{a}\right)-m^{c}\left(\nabla_{c} n^{a}\right) \chi^{b} \nabla_{b} \chi_{a}-n^{a} m^{c}\left(\nabla_{c} \chi^{b}\right) \nabla_{b} \chi_{a} .
\end{gathered}
$$

De A.2.1 temos que $m^{c} \nabla_{c} n^{a}=-(\beta+\bar{\alpha}) \chi^{a}+\mu m^{a}+\bar{\lambda}_{\bar{m}}^{a}$ e dado que $\chi^{b} \nabla_{b} \chi_{a}=\varkappa \chi_{a}$, é trivial que todos os produtos escalares são identicamente nulos no segundo termo de (3.3.6). Fazendo o mesmo procedimento para o terceiro termo e notando que $\rho=\sigma=0$ vemos que o mesmo é igualmente nulo. Agora usando a relação de Killing $\nabla_{c} \nabla_{b} \chi_{a}=-R_{b a c d} \chi^{d}$ obtemos

$$
m^{c} \nabla_{c} \varkappa=-R_{a b c d} \chi^{a} m^{b} \chi^{c} n^{d}
$$


Para calcular o último termo vamos usar o fato de que $m^{a} \nabla_{a} \rho=0=m^{c} \nabla_{c}\left(-m^{a} \bar{m}^{b} \nabla_{b} \chi_{a}\right)$. Utilizando novamente a relação de Killing

$$
0=m^{c} \nabla_{c}\left(-m^{a} \bar{m}^{b} \nabla_{b} \chi_{a}\right)=R_{d a b c} \chi^{d} m^{a} \bar{m}^{b} m^{c},
$$

decompondo o tensor de Riemann na base da tetrada verificamos então que

$$
0=-R_{d b} \chi^{d} m^{b}+R_{a b c d} \chi^{a} m^{b} \chi^{c} m^{d}
$$

e portanto $m^{a} \nabla_{a} \varkappa=R_{a b} \chi^{a} m^{b}$, ou utilizando as equações de Einstein $m^{a} \nabla_{a} \varkappa=8 \pi T_{a b} \chi^{a} m^{b}$. A condição de energia dominante implica que $T_{a b} \chi^{a}$ deve ser proprocional a um covetor causal. Porém da equação de Sachs temos que se a expansão e o cisalhamento são nulos ao longo do horizonte então $2 \Phi_{00}=R_{a b} \chi^{a} \chi^{b}=0$ e portanto $T_{a b} \chi^{a} \propto \chi_{b}$ o que implica que $T_{a b} \chi^{a} m^{b}=0$ e portanto $m^{a} \nabla_{a} \varkappa=0$. 


\section{Capítulo 4}

\section{O Espaço-Tempo de Kerr}

$\mathrm{O}$ s teoremas sobre a estrutura global de um espaço-tempo que contém um buraco negro desenvolvidos majoritariamente por Penrose e Hawking permitiram o entendimento de que não só os buracos negros são a conclusão inevitável do colapso gravitacional de um corpo muito massivo como também revelaram muito sobre a estrutura dos mesmos, como a formação de singularidades e que durante sua evolução a área de um buraco negro nunca decresce. Com relação ao estágio final supostamente estacionário também mostramos que tal buraco negro possui inúmeras propriedades notáveis, dentre elas que o horizonte de eventos constitui uma superfície algebricamente especial.

A degenerescência algébrica como um caso geral no entanto não foi o caminho histórico. Uma década antes de tal demonstração [23] Roy Kerr $]^{7}$ havia encontrado uma família de buracos negros que possuiam momento angular supondo exatamente se tratar de uma solução algebricamente especial [25]. Esse capítulo é dedicado a explorar tal solução das equações de Einstein e em particular sua relação com um modo de extrair energia de um buraco negro. Ao final teceremos alguns comentários sobre a particularidade de tal solução.

${ }^{*}$ Roy Patrick Kerr (1934- ) 


\subsection{O Giro Inicial}

Vamos aqui passar brevemente pelo caminho que levou Kerr a construir sua métrica, deferindo uma demonstração completa a [24]. Considere uma tetrada nula complexa $\{\mathbf{k}, \boldsymbol{\ell}, \mathbf{m}, \overline{\mathbf{m}}\}$ e sua cotetrada $\left\{\omega^{\mu}\right\}=\{-\ell,-k, \bar{m}, m\}$, onde tomaremos $\mathbf{k}$ como sempre representando um vetor principal nulo [2.4.2]. A métrica nessa base é sempre escrita na forma $d s^{2}=2(m \bar{m}-k \ell)$, que é invariante tanto por transformações de Classe I como de Classe III. Supondo o espaço-tempo ser algebricamente especial temos do teorema de Goldberg-Sachs [2.6.1] que $\kappa=\sigma=0$. Além disso podemos sempre fazer uma rotação de Classe I para tornar $\tau=0$. Assim a forma de conexão [A.1.1] $\boldsymbol{\omega}_{20}=\left(\nabla \mathbf{E}_{0} \mid \mathbf{E}_{2}\right)=(\nabla \mathbf{k} \mid \mathbf{m})$ pode sempre ser escrita como $\omega_{20}=\rho \omega^{3}$.

Para calcular a curvatura utilizamos então a segunda equação estrutural de Cartan A.1.4, de onde $\Omega_{20}=d \omega_{20}-\omega_{20} \wedge\left(\omega_{01}+\omega_{32}\right)$. Fazendo o produto exterior dos dois lados por $\omega_{20}$ obtemos então $\omega_{20} \wedge d \omega_{20}=0$. Essa equação então pode ser vista como uma equação diferencial introduzindo um sistema de coordenadas. O passo seguinte de Kerr foi supor então a existência de dois grupos de isometrias correspondendo a um espaço-tempo estacionário e axissimétrico. Por fim impondo que o espaço-tempo seja também assintoticamente plano ele encontrou uma família de dois parâmetros de métricas [25]. Em particular denotando esses parâmetros por $M$ e $a$ ele mostrou que para $a=0$ temos a métrica de Schwarzschild. No artigo original foram empregadas coordenadas "esféricas". Em coordenadas de Boyer-Lindquist a métrica de Kerr é

$$
\begin{aligned}
d s^{2}= & -\left(1-\frac{2 M r}{\Sigma}\right) d t^{2}-\frac{4 M \operatorname{Mrasen}^{2} \vartheta}{\Sigma} d t d \phi+\frac{\Sigma}{\Delta} d r^{2} \\
& +\Sigma d \vartheta^{2}+\left(r^{2}+a^{2}+\frac{4 M r a \operatorname{sen}^{2} \vartheta}{2 \Sigma}\right) \operatorname{sen}^{2} \vartheta d \phi^{2}
\end{aligned}
$$

onde $\Sigma=r^{2}+a^{2} \cos ^{2} \vartheta$ e $\Delta=r^{2}-2 M r+a^{2}$. É fácil verificar que $\operatorname{det} \mathbf{g}=-\Sigma \Delta \operatorname{sen}^{2} \vartheta$, de modo que é natural se perguntar o que ocorre quando $\Sigma$ ou $\Delta$ são nulos. No caso do primeiro quando tomamos $\Sigma \rightarrow 0$ a curvatura, em particular o escalar de Kretschmann diverge de modo que tal situação corresponde de fato a uma singularidade no mesmo sentido que a singularidade em Schwarzschild. O mesmo não é o caso para $\Delta=0$, onde 
não existem divergências reais. $O$ conjunto de pontos que satisfazem essa condição corresponde a hiperfícies nulas. Se $M^{2}>a^{2}$ e sendo $r_{ \pm}=M \pm \sqrt{M^{2}-a^{2}}$ pode-se verificar que a superfície $r_{+}$corresponde a um horizonte de eventos, e portanto tal solução é um buraco negro. Já a superfície $r_{-}$é um horizonte de Cauchy [4]. No caso em que $M^{2}=a^{2}$ tais superfícies coincidem e denominamos esse caso buraco negro Kerr extremo. Já no caso $M^{2}<a^{2}$ a equação $\Delta=0$ não possui raízes reais de modo que não existe um horizonte de eventos. Assim os observadores em tal espaço-tempo teriam acesso à singularidade $\Sigma=0$, de modo que supondo a conjectura da censura cósmica iremos daqui por diante ignorar esse caso.

Seja agora $\left\{\mathbf{E}_{\mu}\right\}$ a tetrada real tipo-tempo associada às coordenadas de BoyerLindquist para a solução de Kerr. Os buracos negros de Kerr constituem uma solução das equações de Einstein no vácuo, isto é Ric $=0$, de modo que a curvatura é apenas de Weyl. Tome a base de Hodge canônica [2.2.2] para tais coordenadas e através do procedimento dado nas seções $2.2 \mathrm{e} 2.3$ construa o operador linear ${ }_{c x} \mathbb{C}$ em $\Lambda_{c x}^{2}$ associado a curvatura de Weyl. Pode-se ver então que na base canônica do espaço complexificado dos bivetores o operador linear é dado por

$$
[c x \mathbb{C}]=\left[\begin{array}{ccc}
2(I+i J) & 0 & 0 \\
0 & -(I+i J) & 0 \\
0 & 0 & -(I+i J)
\end{array}\right]
$$

onde $I=M r \Sigma^{-3}\left(r^{2}-3 a^{2} \cos ^{2} \vartheta\right)$ e $J=M a \cos ^{2} \vartheta \Sigma^{-3}\left(3 r^{2}-a^{2} \cos ^{2} \vartheta\right)$, de modo que é trivial constatar que o espaço de Kerr constitui um espaço-tempo algebricamente especial de Tipo D [2.4.1]. De acordo com [2.4.4] temos que existem dois vetores principais nulos repetidos, que nessas coordenadas são dados por

$$
\begin{gathered}
\mathbf{k}=\frac{r^{2}+a^{2}}{\Delta} \partial_{t}+\frac{a}{\Delta} \partial_{\phi}+\partial_{r}, \\
\boldsymbol{\ell}=\frac{r^{2}+a^{2}}{2 \Sigma} \partial_{t}+\frac{a}{2 \Sigma} \partial_{\phi}-\frac{\Delta}{2 \Sigma} \partial_{r} .
\end{gathered}
$$

O fato de que o espaço de Kerr se reduz ao de Schwarzschild no caso $a=0$ nos induz a interpretar o parâmetro $M$ como a massa do buraco negro, fato esse que se confirma calculando a massa de Komar para tal métrica. Já no caso $M=0, a \neq 0$ a solução é o 
espaço de Minkowski, algo que pode ser notado de (4.1.2) já que a curvatura se torna identicamente nula. A métrica (4.1.1) nesse caso reflete o espaço de Minkowski em coordenadas adaptadas a uma família de observadores girantes. Calculando então o momento angular de Komar para a métrica de Kerr temos que o mesmo é dado por $J=M a$, de modo que a solução encontrada por Kerr representa um buraco negro com momento angular não nulo.

\subsection{Processos Dinâmicos em Buracos Negros}

Mostramos na seção anterior que a métrica de Kerr representa uma generalização da métrica de Schwarzschild para o caso de um buraco negro com momento angular. Todos os resultados do capítulo 3 se aplicam devidamente neste caso, em especial aqueles referentes ao estado final. Assim tomamos o espaço de Kerr por representar um buraco negro estacionário axissimétrico produto do estado final do colpaso gravitacional que forma um buraco negro após o mesmo emitir radiação gravitacional. Aqui discutiremos propriedades da solução de Kerr que não estão presentes no caso estático.

Proposição 4.2.1 Seja $(\mathcal{M}, \mathbf{g})$ um espaço-tempo fortemente assintoticamente previsível que contenha um buraco negro estacionário mas não estático, isto é o campo de Killing relacionado a isometria temporal $\xi$ não é ortogonal a hiperfícies. Então o subconjunto de $J^{+}\left(\mathscr{I}^{-}\right) \cap J^{-}\left(\mathscr{I}^{+}\right)$ onde o campo vetorial そ̇é tipo-espaço é denominado ergosfera.

Em particular no caso de Schwarzschild não há ergosfera. Já para a métrica de Kerr não-extrema temos que

$$
(\xi \mid \xi)=\frac{a^{2} \operatorname{sen}^{2} \vartheta-\Delta}{\Sigma}>0 \quad, \quad r^{2}+a^{2} \cos ^{2}-2 M r<0
$$

de modo que a região $r_{+}<r<M+\sqrt{M^{2}-a^{2} \cos ^{2} \vartheta}$ corresponde à ergosfera. A importância de tal região foi levantada por Penrose [26] quando ele mostrou que podese utilizar de tal região para extrair energia de um buraco negro. Considere uma partícula inicialmente assintoticamente distante do buraco negro com momento $\mathbf{p}_{o}$ de 
modo que sua energia (assim como medida por um observador seguindo as isometrias de $\xi$ onde o mesmo é normalizado) é $E_{o}=-\left(\mathbf{p}_{o} \mid \xi\right)>0$. Agora deixemos que tal partícula caia em direção ao buraco negro e que quando a mesma se encontre na ergosfera decaia em duas outras partículas de momentos $\mathbf{p}_{A}$ e $\mathbf{p}_{B}$. O ponto central consiste em perceber que uma vez na ergosfera o fato de que o vetor de Killing $\xi$ é tipo-espaço permite que uma das partículas, por exemplo $A$, tenha energia negativa, isto é $E_{A}=-\left(\mathbf{p}_{A} \mid \xi\right)<0$. Por conservação de energia temos necessariamente que $E_{B}>E_{0}$. A partícula $A$ não pode deixar a ergosfera pois isso implicaria em uma violação da positividade da energia, porém ela pode seguir o caminho contrário e atravessar o horizonte de eventos. Nesse caso se o decaimento ocorrer de forma precisa é possível que a partícula $B$ saia da ergosfera em direção ao infinito com energia maior que a partícula inicial.

Em um espaço-tempo assintoticamente simples e estacionário temos que a massa se conserva, de modo que o buraco negro tem sua massa reduzida de $\left|E_{A}\right|$. Em outras palavras o buraco negro realiza trabalho sobre a partícula incidente de modo que seus produtos ganhem energia às custas da energia do buraco negro. Tal procedimento para extrair energia de um buraco negro é denominado processo de Penrose. Antes de explorar os processos de Penrose no espaço de Kerr cabem alguns comentários. A priori ao introduzirmos uma partícula no espaço-tempo estaremos quebrando sua axissimetria de modo que não deveríamos poder nos utilizar dos campos de Killing. No entanto considerando uma situação em que a energia e momento angular da partícula sejam muito menores que os do buraco negro podemos considerar o processo como uma pequena perturbação e os resultados assim obtidos devem fornecer uma ilustração qualitativamente correta do fenômeno. Para ser preciso vamos considerar que inicialmente a partícula se encontra no infinito e cai no campo gravitacional adiabaticamente e estaremos interessados apenas na configuração final após a partícula emitida novamente se encontrar no infinito e o buraco negro voltar a uma configuração dada pela métrica de Kerr.

Como já adiantado na frase anterior em geral a partícula carregará momento angular também. Dado que para um corpo isolado sempre podemos escolher um referencial 
de modo que seu momento angular seja positivo vamos aqui escolher o campo de Killing cíclico $\varphi$ de modo que o momento angular do buraco negro $J$ seja positivo. Examinaremos assim a influência sobre este da partícula que adentra o horizonte de eventos. Quando a partícula $A$ produto do decaimento está no horizonte temos (dado que o campo de Killing $\chi$ é sempre dirigido ao futuro no horizonte)

$$
0<-\left(\mathbf{p}_{A} \mid \chi\right)=-\left(\mathbf{p}_{A} \mid \xi\right)-\Omega\left(\mathbf{p}_{A} \mid \boldsymbol{\varphi}\right)=E_{A}-\Omega L_{A}
$$

e podemos verificar facilmente que para a solução de Kerr $\Omega=\frac{a}{a^{2}+\left[M+\sqrt{M^{2}-a^{2}}\right]}$ que conforme definimos é positiva. Assim, trivialmente, verifica-se que $L_{A}<E_{A} / \Omega$ e que portanto se $E_{A}<0$ temos que $L_{A}<0$. Portanto a partícula que atravessa o horizonte em um processo de Penrose nessas condições não só diminui a massa como o momento angular do buraco negro. Da equação (4.2.1) constatamos que ambos os efeitos terão como resultado típico a redução da ergosfera e portanto da capacidade de se retirar energia do buraco negro. Para avaliar o quanto se alteram os parâmetros do buraco negro temos de resolver a equação $d M=\Omega d J$, onde consideramos o caso extremo onde vale a igualdade para determinar quanta energia pode ser extraída. Integrando tal equação Christodoulou [27] mostrou que existe uma quantidade $M_{i r r}$ denominada massa irredutivel tal que todos os processos de Penrose devem obedecer $\delta M_{\text {irr }} \geq 0$. Da integração da equação temos que a expressão para a massa irredutível é

$$
M_{i r r}^{2}=\frac{1}{2}\left[M^{2}+\sqrt{M^{4}-J^{4}}\right]
$$

que invertendo podemos escrever

$$
M^{2}=M_{i r r}^{2}+\frac{J^{2}}{4 M_{i r r}^{2}}
$$

que ilustra a razão do nome massa irredutível dado que $M \geq M_{i r r}$ e sendo $\delta M_{i r r} \geq 0$ temos que a massa irredutível reflete a parcela da massa que nunca pode ser convertida em trabalho. Em particular Christodoulou mostrou que os processos para os quais $\delta M_{i r r}=0$ são reversíveis no sentido de que existe um processo de Penrose associado *Demetrios Christodoulou (1951- ). Quando da publicação de [27| ele tinha 19 anos e fazia seu doutorado sob a orientação de Wheeler. 
que restaura o buraco negro de Kerr aos parâmetros iniciais. Por outro lado quando a massa irredutível cresce os processos são irreversíveis, ou seja não existe processo que retorne o buraco negro a configuração inicial.

Podemos então definir a eficiência (máxima) dos processos de Penrose por $\eta=\frac{M-M_{i r r}}{M}$, isto é a razão entre a energia disponível e a energia total. Supondo que o buraco negro encontra-se inicialmente no caso extremo $J=M^{2}$ podemos calcular que a energia máxima disponível é de $\eta=1-1 / \sqrt{2} \approx 29 \%$. O mesmo raciocínio também permite avaliar a energia disponível para ser emitida por ondas gravitacionais resultante da colisão de buracos negros. Em particular considere dois buracos negros de Schwarzschild inicialmente muito distantes que colidem frontalmente até formarem um único buraco negro estático no fim. Então a energia emitida em ondas gravitacionais é também da ordem de $29 \%$ da massa inicial total [17].

Antes de encerrar esta seção queremos encontrar um expressão para a massa explicitamente em termos do momento angular e de eventualmente outros parâmetros. Em um espaço-tempo assintoticamente simples estacionário a massa de Komar é constante e portanto podemos calcular a integral não necessariamente sobre o infinito como também sobre o horizonte de eventos. Vamos aqui utilizar a notação de índices abstratos por simplicidade.

$$
M=-\frac{1}{8 \pi} \int_{\mathscr{C}} \epsilon_{a b c d} \nabla^{c} \xi^{d}=-\frac{1}{8 \pi} \int_{\mathscr{C}} \epsilon_{a b c d} \nabla^{c} \chi^{d}+\frac{\Omega}{8 \pi} \int_{\mathscr{H}} \epsilon_{a b c c} \nabla^{c} \varphi^{d} .
$$

O segundo termo é duas vezes o momento angular de Komar. Precisamos então avaliar o primeiro termo. Para tal vamos novamente utilizar o vetor $\chi^{a}$ para formar uma tetrada nula, onde o outro vetor nulo é dado por $n^{a}$. Então o elemento de volume de $\mathscr{H}$ é dado por $\epsilon_{a b}=\epsilon_{a b c d} n^{c} \chi^{d}$. Vamos então escrever a forma de volume como $1 / 2 \epsilon_{a b} \epsilon^{e f} \epsilon_{a b c d} \nabla^{c} \chi^{d} \mathrm{e}$ avaliar uma parte dela:

$$
\epsilon^{a b} \epsilon_{a b c d} \nabla^{c} \chi^{d}=n_{e} \chi_{f} \epsilon^{a b e f} \epsilon_{a b c d} \nabla^{c} \chi^{d}=-4 n_{c} \chi_{d} \nabla^{c} \chi^{d}=-4 \varkappa
$$

onde $\varkappa$ é a gravidade superficial [3.3.3]. Dado que a gravidade superficial é constante no horizonte podemos tirar esse termo para fora da integral restando $\int_{\mathscr{H}} \epsilon_{a b}$. Sendo $\epsilon_{a b}$ 
a forma de volume do horizonte de eventos então a integral é por definição a área do horizonte $A$ resultando então na fórmula de Smarr

$$
M=\frac{\varkappa}{4 \pi} A+2 \Omega J
$$

No caso de Kerr temos que a gravidade superficial é

$$
\varkappa=\frac{\left(M^{2}-a^{2}\right)^{1 / 2}}{2 M\left[M+\sqrt{M^{2}-a^{2}}\right]},
$$

e a área do horizonte de eventos pode ser calculada diretamente resultando em $A=$ $8 \pi\left[M^{2}+\sqrt{M^{4}-J^{2}}\right]$. Comparando com 4.2 .3 temos que $M_{i r r}=\left(\frac{A}{16 \pi}\right)^{1 / 2}$ e portanto o fato da massa irredutível ser não-decrescente é um caso particular do teorema da área de Hawking [3.2.9].

\subsection{Unicidade e Calvície}

A solução de Kerr tem inúmeras propriedades interessantes devido a axissimetria, isto é devido ao momento angular não nulo. A presença da ergosfera em espaços-tempo estacionários pode ser deduzida sem supor uma solução particular, mas a existência da massa irredutível certamente torna a discussão muito mais rica. Cabe então perguntar o quão geral é supor que um buraco negro estacionário é uma solução de Kerr. Primeiramente note que no caso axissimétrico não existe um análogo ao teorema de Birkhoff [17], de modo que não é verdade que o espaço-tempo exterior a uma estrela girando possa necessariamente ser descrito pela solução de Kerr.

Para o caso estático segue do teorema de Birkhoff que um buraco negro deve ser representado pela métrica de Schwarzschild. Para o caso estacionário temos um teorema devido a Carter [17].

Teorema 4.3.1 Seja $(\mathcal{M}, \mathbf{g})$ um espaço-tempo quadridimensional fortemente assintoticamente previsível estacionário com $\mathrm{Ric}=0$ e axissimétrico. Além disso suponha que a intersecção do horizonte de eventos passado $\dot{J}^{+}\left(\mathscr{I}^{-}\right) \cap \mathcal{M}$ com o horizonte de eventos futuro $\dot{J}^{-}\left(\mathscr{I}^{+}\right) \cap \mathcal{M}$ é uma superfície bidimensional compacta e conexa. Então $\mathbf{g}$ pertence a uma família de métricas 
de dois parâmetros que podemos tomar por sendo a massa Me o momento angular J.

Similarmente Robinson demonstrou [28] que se permitirmos que a métrica seja uma solução de vácuo das equações de Einstein-Maxwell então a métrica pertence a uma família de 3 parâmetros, sendo o terceiro a carga elétrica do espaço-tempo. Esses teoremas levaram Wheeler a propor a conjectura de "No-Hair", a saber que todos os buracos negros estacionários são representados por um número finito de parâmetros, em especial pela métrica de Kerr, ou a de Kerr-Newman para o caso carregado.

A questão da unicidade da métrica de Kerr é até hoje um tópico ativo da pesquisa em Relatividade Geral. Vamos aqui apenas citar alguns pontos que permanecem ainda em aberto. Em primeiro lugar existe o papel do horizonte ser conexo. Para o caso de buracos negros carregados mas sem momento angular existe o exemplo da métrica de Majumdar-Papapetrou [28] de $N$ buracos negros dispostos espacialmente tal que a repulsão elétrica equilibre a atração gravitacional, fornecendo então um contra-exemplo para o caso não conexo. No entanto para o caso de dois buracos negros com momento angular tais que o efeito da interação spin-spin mantivesse a configuração estacionária não é possível [29].

A partir da década de 80 a maior parte do interesse na área mudou para investigar a questão na presença de campos não-abelianos, buracos negros em mais dimensões e na presença da constante cosmológica. Existem exemplos na presença de campos de Yang-Mills que violam a conjectura de "No-Hair" [30], além do fato que para dimensões $N>4$ do espaço-tempo não vale o resultado citado na seção 3.2 de que a topologia deve ser a da esfera, em particular se conhecem soluções em $N=5$ que são topologicamente um toro. Assim a maior parte dos esforços se concentram em encontrar quais são as condições para as quais um buraco estacionário deve satisfazer a conjectura de Wheeler.

No entanto cabe notar que nas condições do teorema [4.3.1] Bunting e Mazur demonstraram que se o horizonte de eventos for um horizonte de Killing não-degenerado, ou seja $\varkappa \neq 0$ então necessariamente a métrica é a de Kerr [30]. Assim trabalharemos com tais condições em mente, guardando para a seção 5.3 a discussão do caso extremo 
em mais detalhes. 


\section{Capítulo 5}

\section{Termodinâmica de Buracos Negros}

o longo dos últimos capítulos estabelecemos o que são buracos negros e qual
é a dinâmica de tais objetos. No final da década de 60 do século XX duas abordagens de espíritos distintos surgiram concomitantemente (sendo que houve uma interação não-nula entre os participantes de ambas). Na Inglaterra, com a introdução de métodos de geometria diferencial, foram desenvolvidos teoremas gerais sobre o colapso gravitacional e buracos negros, culminando no teorema de área de Hawking. Do outro lado do Atlântico o foco residia na solução encontrada por Kerr para um buraco negro com rotação (e posteriormente com carga elétrica), e em procesos dinâmicos para extrair energia do buraco negro utilizando a ergosfera. Veremos agora como essas linhas de pesquisa levaram ao entendimento de que os buracos negros constituem objetos termodinâmicos.

\subsection{Bekenstein e Hawking}

Um buraco negro consiste de uma região do espaço-tempo tal que todas as curvas causais que dela saem não tem como ponto limite o infinito futuro nulo $\mathscr{I}^{+}$. Em outras palavras um observador assintoticamente distante de tal objeto não tem acesso ao que ocorre dentro do mesmo. Com isso em mente surge a possibilidade de violar a segunda lei da termodinâmica, a saber poderíamos despejar entropia dentro de um 
buraco negro e assim reduzir a entropia acessível a um observador assintótico. No entanto Bekenstein] [31] já tinha ciência do resultado devido a Christodoulou de que um buraco negro de Kerr nunca pode ter sua área reduzida através de processos de Penrose [27]. Posteriormente o teorema de Hawking [17] garantia que a área de um buraco negro qualquer nunca diminui dado que se satisfaça condições de energia apropriadas. A semelhança de tais fatos com a segunda lei da termodinâmica suscita a idéia de que atribuindo ao buraco negro uma entropia proporcional a sua área seria possível salvar a segunda lei da termodinâmica: a perda de entropia do objeto regular a ser engolido seria compensada pelo aumento da área.

Ainda no contexto da solução de Kerr, partindo da fórmula de Smarr $M=\frac{\varkappa}{4 \pi} A+2 \Omega J$, e considerando as configurações finais acessíveis via processos de Penrose mostramos (4.2.4) que existe uma quantidade de variação não-negativa denominada massa irredutível que se relaciona com a área por $M_{i r r}=\left(\frac{A}{16 \pi}\right)^{1 / 2}$, o que não só é um caso particular do teorema de Hawking, mas também representa a fração de energia que não pode ser extraída de um buraco negro de Kerr. Em específico a energia que pode ser extraída de um buraco negro de Kerr via processos de Penrose é igual a $M-M_{i r r}$. Desse modo fica claro que a área de fato representa uma degradação de energia, ou em outras palavras energia não acessível para realizar trabalho. Se o experimento mental para violar a segunda lei da termodinâmica não oferecesse evidências suficientes para atribuir entropia a um buraco negro, considerando que poderiam haver outras formas de se evitar tal violação, a associação da massa irredutível com o conceito de calor, embora em um caso particular, é demasiadamente sugestiva para ser ignorada.

Note no entanto que embora a hipótese de que um buraco negro possua uma entropia proporcional à sua área seja não só muito bem motivada pela questão da massa irredutível como também necessária para impedir a violação da termodinâmica, o mesmo não pode ser considerado, do ponto de vista clássico, um sistema termodinâmico. Isso porque não existe uma temperatura física associada ao mesmo para que ele possa interagir com um outro sistema.

${ }^{*}$ Jacob David Bekenstein (1947- ) 
O cenário muda quando levamos em consideração efeitos quânticos sobre o buraco negro. Em especial vamos discutir o mesmo sob o ponto de vista da Teoria Quântica de Campos em Espaços Curvos, isto é os campos de matéria são tratados quanticamente porém a interação gravitacional ainda é clássica. Esse tratamento deve ser apropriado em um regime de baixas energias de modo análogo a produção de pares em um campo eletromagnético (clássico) intenso. Com isso vamos considerar o colapso gravitacional resultando em um buraco negro. Em um espaço-tempo assintoticamente plano podemos descrever os campos, por exemplo, em termos dos modos de frequência positiva "ingoing". No caso do colapso, podemos escolher que os campos estejam no seu estado de vácuo inicialmente, definido como o estado que se torna nulo pela aplicação dos operadores de aniquilação desses modos. Podemos entretando representar os campos em termos dos modos "outgoing" de frequência positiva. Dada a condição inicial de vácuo com relação aos modos "in" cabe a pergunta qual será o estado final após o colapso e formação do buraco negro. Como o espaço-tempo não é estacionário o vácuo "in" é levado em uma combinação de modos "out" que tem média do operador número não-nula. Desse modo os observáveis como medidos por observadores estáticos representam partículas criadas durante o colapso e emitidas para $\mathscr{I}^{+}$.

Em 1973 Zel'dovich ${ }^{\boxplus}$ e seu aluno Starobinsky $\left.\right|^{\dagger}$ mostraram que no caso de um buraco negro com momento angular tal efeito levaria à criação de partículas que carregariam o momento angular de modo que assintoticamente o mesmo parasse de girar. Com isso em mente Hawking [32,33] mostrou que mesmo no caso do colapso esfericamente simétrico existe emissão de partícula, e que ainda mais, no caso de um campo escalar, estas tem média do operador número $\langle N\rangle \propto \frac{e^{-2 \pi \omega / \varkappa}}{1-e^{-2 \pi \omega / x}}$, ou seja obecedem a distribuição de Bose-Einstein. Conversamente um campo de spin $1 / 2$ tem espectro com estatística de Fermi-Dirac. Tomando as unidades geométricas usuais e a constante de Boltzmann como a unidade é trivial de ver que a temperatura associada aos campos é igual a $T=\frac{\hbar \varkappa}{2 \pi}$. Esse efeito, a radiação Hazwking, permite então definitivamente associar uma temperatura física a um buraco negro, o que conjuntamente com a indentificação da

${ }^{\dagger}$ Alexei Starobinsky (1948- ) 
área com entropia devido a Bekenstein, e o teorema de Hawking, sugere fortemente que os buracos negros são de fato objetos termodinâmicos plenamente.

\subsection{As Leis da Mecânica de Buracos Negros}

No âmbito da discussão da seção anterior temos agora justificativa para tratar um buraco negro como um sistema termodinâmico pleno, no sentido que a um estado estacionário podemos associar uma temperatura física, através da emissão de radiação com espectro de corpo negro, e também uma entropia, isto é uma quantidade que indica a limitação do sistema em realizar trabalho. Considerando os resultados discutidos nos capítulos 3 e 4 queremos então mostrar que não só podemos atribuir quantidades que operam analogamente, do ponto de vista físico, às termodinâmicas como também satisfazem relações matemáticas idênticas às leis da termodinâmica em determinados contextos.

\section{A LEI ZERO}

A lei zero da termodinâmica estabelece que dados três sistemas $A, B$ e $C$, se $A$ está em equilíbrio com $B$ e $B$ em equilíbrio com $C$ então $A$ está em equilíbrio com $C$. Além disso se $A$ e $B$ estão em equilíbrio então suas variáveis devem satisfazer um vínculo, a saber que suas temperaturas sejam idênticas. Através da radiação Hawking somos capazes de atribuir uma temperatura, que sabemos corresponder as propriedades físicas desejáveis de uma temperatura, que é proporcional a sua gravidade superficial $\varkappa$. Satisfazem então os buracos negros a definição de lei zero mencionada?

Certamente não, afinal trivialmente dados dois buracos negros quaisquer, suficientemente separados para que possamos considera-los inicialmente estacionários, se ambos tiverem a mesma gravidade superficial isso não os impedirá de eventualmente colidir graças a atração gravitacional. O mesmo se daria trocando um dos buracos negros por um outro tipo de sistema termodinâmico típico, como um gás ideal. O único caso que parece satisfazer tal definição seria considerar um universo preenchido 
com, por exemplo, um gás de fótons a uma dada temperatura $T$. Se o buraco negro possuir gravidade superficial tal que $T=\frac{\hbar \varkappa}{2 \pi}$ então embora parte do gás atravesse o horizonte de eventos sendo absorvido a emissão da radiação Hawking na mesma taxa manterá o sistema em equilíbrio. Interessantemente tal situação viola a condição de que o espaço-tempo seja fracamente assintoticamente simples dado que o tensor energia-momento não decai assintoticamente para o infinito. Nesse caso não temos uma definição rigorosa do que constitui um buraco negro.

A questão reside, é claro, na gravidade. Para que a temperatura satisfaça as propriedades enunciadas acima é necessário que possamos introduzir paredes adiabáticas/diatérmicas na nossa teoria. No entanto a gravitação não só é uma interação de longo alcance como não pode ser blindada de modo análogo ao eletromagnetismo. Não deveríamos então esperar que dois sistemas distintos com a mesma temperatura estivessem em equilíbrio. Parece então que a única esperança seria formular uma lei para um sistema isolado. Um sistema termodinâmico em equilíbrio possui uma temperatura que é única ao longo do sistema. Note que tal proposição supõe um contexto hidrodinâmico, dado que do ponto de vista do formalismo típico se desconsidera a extensão do sistema e a temperatura é uma quantidade que só pode ser definida no equilíbrio. Assim afirmar que a temperatura de um sistema é constante ao longo de um sistema em equilíbrio é em certo sentido redundante. Não obstante tomaremos a lei zero da dinâmica dos buracos negros por:

Seja B um buraco negro estacionário e « sua gravidade superficial. Então a temperatura do buraco negro é dada pela temperatura da radiação Hawking, a saber $T=\frac{\hbar \varkappa}{2 \pi}$, e é constante ao longo do horizonte de eventos.

\section{A PRIMEIRA LEI}

A primeira lei da termodinâmica afirma que a energia total se conserva, na medida em que a variação da energia interna é sempre igual a troca de calor mais a quantidade de trabalho realizada sob o sistema. Em particular dada a definição usual de entropia obtemos formalmente que $\delta U=T \delta S+\delta W$. Como discutido previamente sabemos que a gravidade superficial corresponde a temperatura e postulamos que a área é 
proporcional a entropia. Mais ainda a massa do buraco negro reflete a sua energia, e a presença de momento angular dá margem a realização de trabalho por processos de Penrose. Vamos então indicar uma demonstração da primeira lei da mecânica dos buracos negros, que por ser longa reservamos os detalhes para [23], utilizando a notação de índices abstratos. Forneceremos uma demonstração distinta posteriormentre no capítulo6.

Tome a fórmula de Smarr 4.2.7), e considere que fazemos uma perturbação no buraco negro induzindo uma variação

$$
\delta M=\frac{1}{4 \pi}(\delta \varkappa A+\varkappa \delta A)+2(\delta \Omega J+\Omega \delta J) .
$$

Vamos considerar que essa perturbação seja tal que $\delta \xi^{a}=\delta \varphi^{a}=0^{\ngtr}$ e portanto $\delta \chi^{a}=$ $\delta \Omega \varphi^{a}$. Agora considere dois vetores $X^{a}$ e $Y^{a}$ tais que $\nabla_{a} X^{a}=\nabla_{a} Y^{a}=0$ e $[X, Y]^{a}=$ 0 . Então é fácil de verificar que $\nabla_{a}\left(X^{[a} Y^{b]}\right)=0$. Esse resultado pode ser entendido trivialmente no caso tridimensional. Dados dois vetores temos que o bivetor $X^{[a} Y^{b]}$ pode ser naturalmente identificado com o produto vetorial de $X^{a}$ por $Y^{a}$. A afirmação então constitui que o rotacional do produto vetorial de dois vetores de divergente nulo e que comutam é nulo, o que é fácil verificar da identidade vetorial para o rotacional do produto vetorial e de que a afirmação de que eles comutam equivale a $\nabla_{X} Y=\nabla_{Y} X$. Agora sendo o bivetor pertencente ao espaço $\Lambda^{2}$ podemos utilizar o dual de Hodge [2.2.3], que é um isomorfismo entre $\Lambda^{p}$ e $\Lambda^{N-p}$ e portanto leva de $\Lambda^{2}$ nele mesmo, para mostrar que $\nabla_{[a}\left(\epsilon_{b c] d e} X^{d} Y^{e}\right)=0$.

Agora tomemos uma hiperfície assintoticamente simples $C$ que intercepta o horizonte de eventos em uma 2-esfera . No espaço-tempo do buraco negro tal hiperfície tem como fronteira $\partial C=\mathscr{H} \cup S$ onde $S$ representa uma esfera no infinito. Integrando a expressão anterior na superfície de Cauchy temos

$$
\int_{C} \nabla_{[a}\left(\epsilon_{b c] d e} X^{d} Y^{e}\right)=0
$$

* Para a existência de uma perturbação que não altere os campos de Killing e o horizonte de eventos veja [23]. Para o caso de um espaço-tempo vácuo isso decorre do fato de que todos os buracos negros são tipo Kerr $|4|$. 
que por virtude do teorema de Stokes equivale a

$$
\int_{S} \epsilon_{b c d e} X^{d} Y^{e}=\int_{\mathscr{H}} \epsilon_{b c d e} X^{d} Y^{e} .
$$

Para encontrar a primeira lei da mecânica dos buracos negros basta uma escolha judiciosa de vetores. Vamos tomar $X^{d}=\xi^{d}$ e $Y^{c}=\nabla_{e}\left(h^{c e}-g^{c e} h\right)$ onde $h_{a b}$ é a perturbação na métrica e $h$ o seu traço. Então o lado esquerdo de 5.2 .3 é $\int_{S} \epsilon_{a b c d} \xi^{c} \nabla_{e}\left(h^{d e}-g^{d e} h\right)=8 \pi \delta M[4]$. Para calcular o lado direito precisamos utilizar o fato de que $£_{\chi} \delta \chi^{a}=0$ e supor que $\delta \chi_{a} \propto$ $\chi_{a}$ no horizonte de eventos ${ }^{\ngtr}$ o que permite mostrar que $\delta \varkappa=-\frac{1}{2} \nabla^{a}\left(\delta \chi_{a}\right)+\delta \Omega \chi^{a} n^{b} \nabla^{b} \varphi_{a}$ [23] e disso temos que

$$
8 \pi \delta M=-2 \delta \varkappa A-16 \pi \delta \Omega J
$$

que substituindo em (5.2.1) nos leva a:

Seja B um buraco negro estacionário sujeito a uma perturbação também estacionária, inicialmente de massa $M$, momento angular J e área A. Então a variação desses parâmetros devido tal perturbação é dada por

$$
\delta M=\frac{\varkappa}{8 \pi} \delta A+\Omega \delta J
$$

Além do método aqui sugerido, que segue a derivação original de Bardeen $\overbrace{}^{\dagger}$, Carter $\overbrace{}^{\ddagger}$ e Hawking, pode-se demonstrar a primeira lei variando diretamente os parâmetros da métrica de Kerr [34] ou então considerando a variação devido ao fluxo de energiamomento pelo horizonte de eventos [35], além é claro do método da carga de Noether discutido no capítulo 6. Cabe notar que dada a equação (5.2.5) e a expressão da radiação Hawking podemos então determinar a constante de proporcionalidade entre a entropia e a área de modo que a variação seja $T \delta S$ e portanto temos que $S=\frac{A}{4 \hbar}$.

\section{A SEGUNDA LEI}

A segunda lei da termodinâmica pode ser resumida de modo muito sintético pela proposição de que qualquer processo termodinâmico deve sempre obedecer o vínculo

\footnotetext{
*Tal relação decorre de escolhermos a mesma posição para os horizontes de eventos da solução inicial e da perturbada [23].

†James Maxwell Bardeen (1939- )

‡Brandon Carter (1942- )
} 
$\delta S \geq 0$. Aqui é notória que ao passo que a lei zero deve ser encarada em um contexto mais restrito que o usual e a primeira lei é válida sem mais reservas do que as termodinâmicas, o teorema de Hawking [3.2.9] garante a validade da segunda lei para o caso do buraco negro de modo muito mais extenso. Perceba que no caso termodinâmico é possível que a entropia diminua em processos fora do equilíbriof o crescimento dela sendo uma afirmação apenas sobre os estados de equilíbrio. Já no caso do buraco negro o aumento da área pode ser demonstrado sem recorrer a existência de uma isometria temporal, ou seja permanece válida até mesmo durante os instantes em que não podemos tratar o buraco negro como um estado de equilíbrio. Lembrando a discussão devido a Bekenstein da seção 5.1 vamos então tomar a segunda lei para buracos negros como

Seja B um buraco negro e A sua área, a entropia do buraco negro é proporcional a área e, denotanto por $S_{m}$ a entropia devido a matéria no exterior do buraco negro, em qualquer processo a desigualdade $\delta\left(S+S_{m}\right) \geq 0$ é sempre válida.

Perceba que essa desigualdade permite inclusive que a entropia do buraco negro diminua, desde que compensada pelo crescimento recíproco da entropia da matéria. Esse é o caso particular da radiação Hawking, que ao fornecer energia ao infinito diminui a massa do buraco negro e portanto sua área. Nesse caso a entropia da radiação emitida compensa o decréscimo da área do buraco negro. A violação do teorema de Hawking se deve ao fato de que campos quânticos violam a condição de energia forte, necessária para demonstrar o teorema.

\subsection{O Postulado de Nernst}

Estabelecido que buracos negros satisfazem a primeira e segunda lei da termodinâmica de modo preciso e a lei zero até certo ponto, sendo a discrepância atribuída a natureza da gravitação ser uma interação de longo alcance e que não pode ser blindada, é natural então que nos perguntemos sobre o status da terceira lei da termodinâmica, também *Aqui nos referimos a entropia fora do equilíbrio no sentido de Boltzmann, isto é $S=k_{B} \ln W$. 
conhecida como postulado de Nernst? para o caso dos buracos negros. No entanto ao passo que as primeiras leis formam consenso no que tange sua generalidade a terceira lei não goza do mesmo patamar, tendo sido descrita como algo não fundamental ou então como se aplicando apenas a casos particulares. Para evitar ambiguidades vamos discutir brevemente o que tomamos pela terceira lei em si antes de investigá-la para o caso dos buracos negros.

A primeira formulação da $3^{a}$ lei da termodinâmica é conhecida como enunciado de Nernst e consiste da afirmação de que $\lim _{T \rightarrow 0} \Delta S=0$ ou equivalentemente que a isoterma $T=0$ é também uma curva adiabática. A modificação de tal enunciado é devido a Planck $\mathrm{f}^{\dagger}$ e decorre de notar que do modo como posto por Nernst existe a possibilidade de que $\lim _{T \rightarrow 0} S=-\infty$. Portanto o que hoje é tomada usualmente por lei é o enunciado de Planck que consiste do enuciado de Nernst suplementado pela condição de que $\lim _{T \rightarrow 0} S=\Xi$ onde $\beth$ é uma constante universal que podemos tomar por sendo zero. Assim a lei poderia ser dada pela afirmação de que a curva isoterma $T=0$ é também a curva adiabática $S=0$. Note que esse enunciado, no contexto da mecânica estatística, pode ser então interpretado como uma proposição sobre o estado fundamental do sistema, a saber que o mesmo é não-degenerado. E embora seja trivial fabricar um contra-exemplo teórico é importante ressaltar que não existe experimento que não tenha indicado a validade da terceria lei como enunciada por Planck.

Agora é evidente que tal formulação não se aplica de modo direto ao caso de um buraco negro, dado que sua área é $A=8 \pi\left[M^{2}+\sqrt{M^{4}-J^{2}}\right]$ 4.2.4 e para o caso extremo temos $J=M^{2}$ do que facilmente se vê que $\varkappa=0$ porém $A \neq 0$. A resolução típica atribuída a tal enigma é adotar a postura de que o enunciado de Planck não é geral por fazer uma afirmação particular sobre o estado fundamental, e então colocar que o enunciado de Nernst equivale a dizer que não existem processos físicos que levem um sistema de uma configuração com temperatura positiva a uma configuração de temperatura nula em tempo finito [4,23]. No entanto a inatingibilidade da temperatura nula de modo algum pode ser deduzida, ou tomada por equivalente, do enunciado 
de Nernst. O que pode sim ser demonstrado decorrer do enunciado de Nernst é que dados dois estados $A$ e $B$ tais que $T(A) \neq 0$ e $T(B)=0$ então não é possível levar o sistema da configuração A para B através de processos reversíveis e adiabáticos [36]. Note que de modo algum podemos equiparar processos físicos, qualquer que seja uma definição razoável de físico, por reversíveis e adiabáticos, a última condição sendo claramente mais restritiva que a primeira. De fato tal condição é tão forte que torna tal afirmação praticamente inútil [36]. É ainda mais interessante a adição usual da expressão "em tempo finito" dado que a termodinâmica não contém o tempo como variável explícita.

No contexto específico dos buracos negros existem ainda contra-exemplos da proposição de que a configuração extrema é inatingível em tempo finito, utilizando por exemplo um buraco negro não extremo envolto por uma casca esférica que quando do seu colapso leva o buraco negro a extremalidade [37]. Vamos no entanto supor que embora não seja decorrente do enunciado de Nernst uma formulação possível para a terceira lei da termodinâmica seja de que uma classe determinada, não extremamente restritiva, de processos não é capaz de levar um sistema da temperatura positiva a temperatura nula em "tempo finito' 7 . Com isso em mente, e o fato de que para o caso de Kerr extremo sempre temos $\theta=0$ para as direções principais nula, tomaremos pela terceira lei da mecânica dos buracos negros o seguinte teorema devido a Israe $]^{\dagger}[38]$ :

Teorema 5.3.1 Seja $(\mathcal{M}, \mathbf{g})$ um espaço-tempo fortemente assintoticamente previsível que contenha um buraco negro e tal que a métrica $\mathbf{g}$ é uma função contínua e seccionalmente de classe $C^{3}$. Denotando uma superfície de Cauchy em um tempo $\tau$ por $S(\tau)$ então se $S(\tau)$ contém uma superfície aprisionada fechada para $\tau<\tau_{1}$ e não contém nenhuma superfície aprisionada fechada para $\tau>\tau_{1}$ então a condição de energia fraca é violada em uma vizinhança do horizonte aparente.

Posta nesta forma fica evidente a relevância da $3^{\text {a }}$ lei para os buracos negros, ela é um caso particular da conjectura da censura cósmica [39] que mencionamos na seção

\footnotetext{
*No caso da Relatividade Geral a definição de tempo finito depende de a qual observador nos referimos. Vamos empregar a expressão em um sentido deliberadamente impreciso, resguardando uma definição apropriada ao teorema de Israel.

${ }^{+}$Werner Israel (1931- )
} 
3.1. Considere que se fosse possível extremalizar um buraco negro em tempo finito então é plausível que uma próxima perturbação poderia formar uma singularidade nua. Uma formulação apropriada da terceira lei consiste então de que a menos de situações patológicas não devemos poder transformar um buraco negro em uma singularidade nua. Aqui cabe colocar que no exemplo devido a Farrugia [37] a patologia reside na casca esférica, dado que podemos usar situações envolvendo o colapso de duas cascas esféricas concêntricas para formar singularidades nuas [20], e portanto essa situação não necessariamente reflete uma falha, mas sim uma das condições a ser imposta. Em segundo lugar o teorema de Israel deixa aberta a possibilidade da formação de singularidades nuas através de violações de condições de energia, algo que sabemos proceder para campos quânticos. O leitor interessado em violações da conjectura da censura cósmica que se utilizam de tais artifícios pode encontrar uma discussão, por exemplo, em [40].

Em vista de que não se conhece exemplo realista de sistema termodinâmico que não satisfaça o enunciado de Planck a situação da solução de Kerr extrema é meritória de maior reflexão. Em particular note que embora a solução de Kerr satisfaça as condições da definição de um buraco negro [3.1.1] ela difere em muito dos buracos negros típicos em que não contém superfícies aprisionadas fechadas e seu horizonte de Killing não é bifurcado, ambos fatos que sugerem que podem existir diferenças topológicas significativas. É aqui importante ressaltar que no caso extremo o horizonte de Cauchy coincide com o horizonte de eventos e sendo o primeiro instável por perturbações não há motivo para não tomar o mesmo para o último. Além disso note que nos casos conhecidos de colapso que forma um buraco negro extremo o espectro de radiação emitido não é térmico [41], de modo que retornando ao raciocínio de Hawking da seção 5.1 não teríamos motivo para atribuir realidade termodinâmica a tal sistema. Levando em conta tais questões poderia-se sugerir que o caso extremo não representa de fato um objeto termodinâmico [41] e que portanto não deveríamos tomá-lo por base para interpretar a terceira lei da mecânica dos buracos negros. Em particular poderiase especular que quando a temperatura do buraco negro é muito baixa, em relação a 
alguma escala dada, possivelmente, pelos parâmetros do buraco negro, então os efeitos de gravitação quântica não podem mais ser ignorados do mesmo modo que um gás de elétrons pode ser descrito por estatística clássica a altas temperatura mas sua natureza quântica é evidente quando a temperatura é menor que a escala típica da superfície de Fermi. Nesse cenário o caso extremo não seria representativo da temperatura nula e em princípio o buraco negro poderia estar de acordo com o enunciado de Planck para a $3^{\text {a }}$ lei da termodinâmica.

\subsection{A Transição de Davies}

Para encerrar esse capítulo vamos discutir algumas questões da termodinâmica da solução de Kerr. Em particular o fato de que os buracos negros satisfazem enunciados que, sob condições particulares específicas, são análogos às leis da termodinâmica de modo algum encerra tudo que há de interessante sobre eles. Aqui vamos partir da equação fundamental para os buracos negros para estudar as transições de fase que ocorrem conforme varia-se o momento angular, seguindo o trabalho seminal de Davies][39].

Fornecida uma equação fundamental $U(S, V)$, que no nosso caso será $M(S, J)$, é natural que exploremos tal equação para melhor compreender o comportamento termodinâmico do sistema. Vamos por simplicidade adotar um sistema de unidades tal que $c=G=\hbar=1$ de modo que a entropia da solução de Kerr é simplesmente $S=A / 4$. Usando a expressão para a massa irredutível (4.2.4) obtemos trivialmente a relação

$$
M(S, J)=\left[\frac{S}{4 \pi}+\pi \frac{J^{2}}{S}\right]^{\frac{1}{2}},
$$

e vemos trivialmente que a massa é uma função homogênea de grau $1 / 2$ ao contrário do grau 1 usual da termodinâmica e portanto não satisfaz a noção de extensividade típica. Esse fato certamente não deve ser encarado como problemático pois sendo a gravitação uma interação de longo alcance não teríamos motivos para esperar a extensividade. 
É fácil verificar a partir da equação 5.4 .1$)$ que $T=\left(\frac{\partial M}{\partial S}\right)_{J}$ e $\Omega=\left(\frac{\partial M}{\partial J}\right)_{S}$ e também que $\frac{\partial M}{\partial S \partial J}=\frac{\partial M}{\partial J \partial S}$ de acordo com o normal. Usando esses resultados e o teorema de Euler para funções homogêneas podemos então deduzir a fórmula de Smarr.

Até aqui nada fora do ordinário. Vamos então calcular a capacidade térmica a momento angular constante (o equivalente a $C_{V}$ ). Temos que

$$
C_{J}=T\left(\frac{\partial S}{\partial T}\right)_{J}=\frac{T M S^{3}}{\pi J^{2}-T^{2} S^{3}}
$$

através da qual é podemos verificar que no caso de $J \rightarrow 0$, isto é na solução de Schwarzschild, a capacidade térmica se torna negativa $C_{J}=-8 \pi M^{2}$. Reescrevendo o capacidade térmica como

$$
C_{J}=\frac{4 T M S}{1-4 T(2 \pi M+T S)^{\prime}}
$$

fica trivial de ver que $\lim _{T \rightarrow 0} C_{J}=0^{+}$e $\lim _{T \rightarrow \infty} C_{J}=0^{-}$e portanto a capacidade térmica troca de sinal, sendo negativo para pequenos momentos angulares e positivo para grandes momentos angulares (tomando a massa fixa). Dado que o numerador consiste do produto de quantidades positivas definidas então a troca de sinal implica em uma descontinuidade do capacidade térmica. Mais ainda é fácil de ver que o denominador pode ser nulo e portanto a descontinuidade é devido a uma divergência, a saber para $J^{2}=(2 \sqrt{3}-3) M^{4}$. A divergência da capacidade térmica assinala a existência de uma transição de fase de $2^{\mathrm{a}}$ ordem, chamada transição de Davies.

A primeira coisa que devemos notar é que a existência de uma fase com capacidade térmica positiva abre a possibilidade que um buraco negro nesta fase possa atingir o equilíbrio com radiação a uma temperatura distinta. Em segundo lugar as transições de fase de $2^{a}$ ordem são usualmente associadas a transições tipo ordem-desordem, como no caso do modelo de Ising para dimensão maior ou igual a 2. No entanto nada aparenta ser distinto entre as fases, a menos da capacidade térmica e outras quantidades associadas com segundas derivadas da equação fundamental. Em particular não é trivial existir nem algo similar a ferromagnetismo-paramagnetismo, onde a questão da ordem é direta, ou algo como o gás de Van der Waals onde embora não seja evidente é possível definir um parâmetro de ordem. 
A ausência do parâmetro de ordem é particularmente desagradável pois através dele podemos calcular os expoentes críticos da transição, que estão fortemente atrelados a aspectos qualitativos da descrição microscópica do sistema, como a dimensionalidade do sistema, a natureza das interações ser de longo ou curto alcance dentre outros. De posse dos expoentes críticos poderíamos inferir aspectos da dinâmica microscópica, em especial comparando com sistemas que dividissem os mesmos expoentes. Mas certamente podemos dizer que dado que a capacidade térmica diverge, de modo polinomial e não logarítmico, então nessa transição temos que $C \propto\left(\frac{T-T_{c}}{T_{c}}\right)^{-\alpha} \operatorname{com} \alpha \neq 0$ e portanto não corresponde a dinâmica da aproximação de campo médio [42]. 


\section{Capítulo 6}

\section{Entropia e Carga de Noether}

1 Ão é infrequente na história da matemática que um mesmo teorema possua duas rema de Euclides da infinitude dos números primos. A primeira demonstração, devido ao próprio Euclides ${ }^{\star}$, envolvia supor que existem finitos primos e então construir explicitamente um número primo não pertencente ao conjunto. Dado que o procedimento é geral isso implica que os primos são infinitos. Posteriormente Euler ${ }^{\dagger}$ forneceu uma demonstração analítica do mesmo teorema [43]. Essa demonstração pavimentou o caminho para a teoria analítica dos números. Assim cabe notar que uma demonstração nova de um fato conhecido pode levar a um entendimento distinto do tópico geral.

Com esse espírito vamos nesse capítulo apresentar uma demonstração distinta da $1^{\text {a }}$ lei da dinâmica de buracos negros devido a Robert Wald $[44]$. Essa demonstração não só tem um valor intrínseco por ser, em algum sentido, mais simples que a original, como também, como discutiremos, elucida o papel decisivo do horizonte de Killing bifurcado e da presença respectiva de um campo de Killing para a existência da $1^{\text {a }}$ lei, além de sugerir um novo modo de entender a relação de tal lei com a dinâmica gravitacional.

\footnotetext{
${ }^{*}$ Euclides de Alexandria (c. $300 \mathrm{AC}$ )

${ }^{\dagger}$ Leonhard Euler (1707-1783)

‡Robert Manuel Wald (1947- )
} 


\subsection{O Potencial Simplético e a Carga de Noether}

No que segue consideraremos um espaço-tempo $(\mathcal{M}, g)^{\circledast}$ quadridimensional com orientação positiva $\omega$. Além disso vamos tomar $(\mathcal{M}, g)$ sendo assintoticamente plano no infinito nulo e espacial [4], uma definição de planicidade assintótica mais forte que assintoticamente simples que garante que os campos tensorias sejam "bem conportados" não só no infinito nulo como no infinito espacial. Essa condição será necessária para garantir a convergência de integrais de campos tensoriais em hiperfícies tipo-espaço. Estaremos interessados em uma formulação lagrangiana para a dinâmica gravitacional, isto é em uma função $L(g)$ da métrica, e possivelmente suas derivadas, tal que obtemos as equações de movimento através das equações de Euler-Lagrange. Em particular iremos trabalhar com a forma diferencial lagrangiana dada por $\mathbf{L} \equiv L \omega$.

Definição 6.1.1 Seja L uma forma diferencial lagrangiana tal que para qualquer difeomorfismo $f: \mathcal{M} \rightarrow \mathcal{M}$ temos que $\mathbf{L}\left(f^{*} g\right)=f^{*} \mathbf{L}(g)$, onde $f^{*}$ denota o "pull back" [4], então $\mathbf{L}$ é uma lagrangiana invariante por difeomorfismos.

Dada uma lagrangiana invariante por difeomorfismos podemos escrever sua variação em um termo de volume e um termo de superfície [45], de modo que denotando a variação por $\delta$ temos $\delta \mathbf{L}=\mathbf{E} \delta g+d \mathbf{\Theta}$, onde $d$ é a derivada exterior [12] e deixamos implícita a contração no termo de volume.

Definição 6.1.2 Seja $\mathbf{L}$ uma lagrangiana invariante por difeomorfismos e $\delta \mathbf{L}=\mathbf{E} \delta g+d \boldsymbol{\Theta}$. Obtemos as equações de movimento tomando $\mathbf{E}=0$ e a 3-forma diferencial $\mathbf{\Theta}(g, \delta g)$ é chamada potencial simplético associado a $\mathrm{L}^{\dagger}$

Definição 6.1.3 Seja $\Theta(g, \delta g)$ o potencial simplético associado a uma lagrangiana invariante por difeomorfismos $\mathbf{L}$ e denotando $\delta$ e ð por variações independentes então a 3-forma diferencial $\boldsymbol{\omega}(g, \delta g, \partial g):=ð \Theta(g, \delta g)-\delta \Theta(g, \partial g)$ 求é a corrente simplética associada $\mathbf{L}$.

\footnotetext{
*Neste capítulo iremos denotar a métrica bem como todos os campos vetoriais por letras em tipografia normal, em contraste com o resto do texto. Reservaremos a tipografia em negrito para as formas diferenciais afim de ficar mais clara a exposição.

${ }^{\dagger}$ Note que reside uma ambiguidade na definição do potencial simplético devido a adição a ele de uma forma fechada. Comentaremos adiante essa ambiguidade.

¥Aqui $ð \boldsymbol{\Theta}(g, \delta g)$ denota avaliarmos $\boldsymbol{\Theta}(g, \delta g)$ e então tomarmos a variação .
} 
Definição 6.1.4 Seja $\boldsymbol{\omega}$ uma corrente simplética associada a uma lagrangiana invariante por difeomorfismos $\mathbf{L}$ e $\mathcal{C}$ uma superfície de Cauchy de $(\mathcal{M}, g)$. Tomemos por orientação de $C$ a forma $n \cdot \omega$, onde $n$ é um vetor normal a $C$ orientado para o futuro. Então $\boldsymbol{\Omega}(g, \delta g, \partial g):=$ $\int_{C} \omega(g, \delta g, \partial g)$ é a forma simplética associada $a \mathrm{~L}^{*}$

Aqui é importante comentar a possível ambiguidade introduzida na forma simplética devido ao fato que o potencial simplético está definido a menos de uma forma fechada, isto é $\boldsymbol{\Theta} \rightarrow \boldsymbol{\Theta}+d \boldsymbol{\Xi}$, onde $\Xi$ é uma 2 -forma qualquer. Tal termo induziria da forma simplética um termo adicional $\int_{\partial C} \partial \Xi(g, \delta g)-\delta \Xi(g, \partial g)$, porém a condição do espaçotempo ser assintoticamente plano no infinito nulo e espacial é suficientemente forte para que esse termo adicional seja identicamente nulo [46]. Assim sendo a lagrangiana L define univocamente a forma simplética a ela associada.

Definição 6.1.5 Seja そ̆ um campo vetorial em $(\mathcal{M}, g)$ a derivada de Lie da métrica em relação a $\xi$ pode ser considerada uma variação da métrica $\hat{\delta} g:=£_{\xi} g$. Sendo $\Omega$ uma forma simplética associada a $\mathbf{L}$, definimos a hamiltoniana conjugada $a \xi, H$, pela sua variação $\delta H:=\mathbf{\Omega}\left(g, \delta g, £_{\xi} g\right)$.

Definição 6.1.6 Seja そ um campo vetorial em $(\mathcal{M}, g)$ e $\boldsymbol{\Theta}(g, \delta g)$ um potencial simplético associado a $\mathbf{L}$, então a corrente de Noether associada a $\xi$ é definida pela 3-forma diferencial $\mathbf{J}\left(g, £_{\xi} g\right):=\boldsymbol{\Theta}\left(g, £_{\xi} g\right)-\xi \cdot \mathbf{L}$.

Proposição 6.1.7 Seja J uma corrente de Noether associada a ł e g uma métrica que satisfaça às equações de movimento $\mathbf{E}=0$, então $\mathbf{J}$ é uma forma diferencial fechada.

Demonstração Tomando a derivada exterior da corrente de Noether temos $d \mathbf{J}=d \mathbf{\Theta}+$ $d(\xi \cdot \mathbf{L})$. Vamos utilizar agora a identidade $£_{\xi} \boldsymbol{\Lambda}=\xi \cdot d \boldsymbol{\Lambda}+d(\xi \cdot \boldsymbol{\Lambda})$ [4]. Note que caso $\Lambda$ seja uma 4-forma, como é o caso da lagrangiana, então compulsoriamente temos $d \boldsymbol{\Lambda}=0$. Desse modo obtemos $d \mathbf{J}=d \mathbf{\Theta}-£_{\xi} \mathbf{L}$ que por definição resulta em $d \mathbf{J}=-\mathbf{E} £_{\xi} g$. Se $g$ satisfaz as equações de movimento então temos que $\mathbf{E}=0$ e portanto $d \mathbf{J}=0$.

\footnotetext{
*Aqui cabe notar que embora a anti-simetria seja garantida por construção para que $\boldsymbol{\Omega}$ constitua uma forma simplética é importante garantir sua não-degenerescência. Uma construção rigorosa pode ser encontrada em [45].
} 
Definição 6.1.8 Seja $\mathbf{J}$ uma corrente de Noether associada a um campo vetorial $\xi$, então segue como corolário da proposição 6.1.7 que existe, ao menos localmente, uma 2-forma $\mathbf{Q}$ tal que $\mathbf{J}=d \mathbf{Q}$. Essa forma é denominada carga de Noether associada a $\xi$.

\subsection{A Primeira Lei da Dinâmica dos Buracos Negros}

Para encontrar a primeira lei da dinâmica dos buracos negros precisamos avaliar a hamiltoniana associada a um campo vetorial em termos da carga de Noether. Nesse intuito precisamos conhecer a variação da corrente de Noether, mantendo fixo o campo vetorial referido

$$
\begin{gathered}
\delta \mathbf{J}=\delta \boldsymbol{\Theta}\left(g, £_{\xi} g\right)-\xi \cdot \delta \mathbf{L}=\delta \boldsymbol{\Theta}\left(g, £_{\xi} g\right)-\xi \cdot[\mathbf{E} \delta g+d \boldsymbol{\Theta}], \\
\delta \mathbf{J}=\delta \boldsymbol{\Theta}\left(g, £_{\xi} g\right)-£_{\xi} \boldsymbol{\Theta}(g, \delta g)-d(\xi \cdot \boldsymbol{\Theta}),
\end{gathered}
$$

onde na última passagem utilizamos as equações de movimento $\mathbf{E}=0$ e a relação para a derivada de Lie de uma forma diferencial previamente utilizada na proposição 6.1.7. É fácil reconhecer em 6.2.2 a expressão para a corrente simplética, de modo que

$$
\boldsymbol{\omega}\left(g, \delta g, £_{\xi} g\right)=\delta \mathbf{J}-d(\xi \cdot \boldsymbol{\Theta})
$$

que integrada sobre uma superfície de Cauchy $C$

$$
\boldsymbol{\Omega}\left(g, \delta g, £_{\xi} g\right)=\delta H=\int_{C} \delta \mathbf{J}-d(\xi \cdot \boldsymbol{\Theta}) .
$$

Proposição 6.2.1 Seja $\boldsymbol{\Omega}\left(g, \delta g, £_{\xi} g\right)$ uma forma simplética que define uma hamiltoniana associada a um campo vetorial $\xi$. Se $\delta g$ satisfaz as equações de movimento linearizadas então a variação do hamiltoniano se reduz a um termo de superfície.

Demonstração No caso em que $\delta g$ satisfaz as equações de movimento linearizadas temos que o comutador $[\delta, d]=0$. Assim sendo temos $\delta \mathbf{J}=\delta d \mathbf{Q}=d \delta \mathbf{Q}$ e portanto utilizando o teorema de Stokes $\delta H=\int_{C} d[\delta \mathbf{Q}-\xi \cdot \boldsymbol{\Theta}]=\int_{\partial C} \delta \mathbf{Q}-\xi \cdot \boldsymbol{\Theta}$. 
Corolário 6.2.2 Seja $\Theta$ um potencial simplético, $\delta H(g, \delta g, \xi)$ a variação de um hamiltoniano associado a um campo vetorial $\xi$ e $\mathbf{Q}$ a carga de Noether associada a mesmo campo vetorial. Se $\delta g$ satisfizer as equações de movimento linearizadas e existir $\mathbf{B}$ tal que $\delta \mathbf{B}=\mathbf{\Theta}$ então o hamiltoniano pode ser escrito explicitamente como $H=\int_{\partial C} \mathbf{Q}-\xi \cdot \mathbf{B}$.

A prova do corolário segue diretamente de substituirmos $\Theta$ por $\delta \mathbf{B}$ na proposição 6.2.1. De posse desse resultado podemos agora fazer as definições que serão relevantes para a primeira lei da dinâmica dos buracos negros.

Definição 6.2.3 Seja L uma lagrangiana invariante por difeormorfismos para a qual o potencial simplético associado satisfaça as condições necessárias para que possamos escrever o hamiltoniano associado a um campo vetorial explicitamente em um termo de superfície. Se o espaço-tempo possuir um campo de Killing t que corresponde a uma isometria temporal então definimos a energia canônica como o valor do hamiltoniano relativo à isometria no infinito espacial $M:=\int_{\infty} \mathbf{Q}(t)-t \cdot \mathbf{B}$. Do mesmo modo caso o espaço-tempo seja axissimétrico com um campo de Killing rotacional $\varphi$, escolhendo $\varphi$ tangente a 2-esfera no infinito, o momento angular canônico é dado por $J:=-\int_{\infty} \mathbf{Q}(\varphi)$.

Proposição 6.2.4 Seja $\mathbf{\Omega}\left(g, \delta g, £_{\xi} g\right)$ uma forma simplética que define uma hamiltoniana associada a um campo vetorial $\xi$. Se $\xi$ for um campo de $\operatorname{Killing}$ de $(\mathcal{M}, g)$ então a forma simplética é indenticamente nula.

A demonstração dessa proposição segue trivialmente da definição do campo de Killing, $£_{\xi} g=0$, e da não-degenerescência de $\boldsymbol{\Omega}$, de modo que $\boldsymbol{\Omega}(g, \delta g, 0)=0$. Passamos agora ao teorema central desta seção.

Teorema 6.2.5 Seja g uma métrica que corresponde a um buraco negro estacionário axissimétrico com um horizonte de Killing bifurcado de normal x e $\delta g$ uma solução estácionária das equações de movimento linearizadas. Denotando a superfície de bifurcação por $\sum$ e sua binormal por $\varepsilon$ então existe um tensor $\mathbf{X}$ tal que se $S:=2 \pi \int_{\Sigma} \mathbf{X}[\varepsilon]$ então

$$
\frac{\varkappa}{2 \pi} \delta S=\delta M-\Omega \delta J
$$

onde $\varkappa$ é a gravidade superficial do buraco negro e $\Omega$ sua velocidade angular. 
Demonstração A forma simplética para o campo de Killing $\Omega\left(g, \delta g, £_{\chi} g\right)$ é nula dada a proposição 6.2.4. Por outro lado da proposição 6.2.1 temos que a variação do hamiltoniano é a integral de superfície $\int_{\partial C} \delta \mathbf{Q}-\chi \cdot \boldsymbol{\Theta}=0$. Tomemos uma hiperfície assintoticamente plana $C$ cuja fronteira seja composta de duas superfícies disjuntas, o infinito espacial $\infty$ e a superfície de bifuracação $\Sigma^{7}$ portanto $\int_{\Sigma} \delta \mathbf{Q}-\chi \cdot \boldsymbol{\Theta}=\int_{\infty} \delta \mathbf{Q}-\chi \cdot \boldsymbol{\Theta}$. Note agora que o campo de Killing é da forma $\chi=t+\Omega \varphi$, onde $t$ é uma translação temporal e $\varphi$ um campo axial. Por construção a carga de Noether é linear no campo vetorial e portanto o lado direito da igualdade é meramente $\delta M+\Omega \delta J$. Lembrando que na superfície de bifurcação o campo de Killing não só tem norma nula como ele mesmo é nulo temos $\int_{\Sigma} \delta \mathbf{Q}(\chi)=\delta M+\Omega \delta J$. Vamos agora expandir a carga de Noether em termos do campo de Killing, porém dado que qualquer derivada do campo de Killing pode ser escrita em termos de $\chi$ e $\nabla \chi]^{\dagger}[4]$ a forma mais geral é $\mathbf{Q}=\mathbf{W}(\chi)+\mathbf{X}(\nabla \chi)$. Novamente na superfície de bifurcação $\chi=0$ e temos apenas o termo de primeira derivada. Finalmente utilizando $\nabla \chi=\varkappa \varepsilon$ e a definição do enunciado obtemos $\frac{\varkappa}{2 \pi} \delta S=\delta M-\Omega \delta J$.

Assim, com uma definição apropriada de entropia, dada por um termo puramente geométrico, podemos facilmente demonstrar a primeira lei da dinâmica de buracos negros. Cabe notar também que as condições do teorema podem ser relaxadas, por exemplo permitindo variações não estacionárias [44, 46]. Além disso o método das cargas de Noether é equivalente a gravitação euclidiana [44]. Nesse contexto é digno de nota que no caso de um buraco negro de Reissner-Nordström extremo (o caso análogo a Kerr extremo com a carga elétrica no lugar do momento angular) a entropia não é proporcional a área e sim nula [49]. Esse resultado é evidente no formalismo da carga de Noether (6.2.5) dado que no caso extremo não existe superfície de bifurcação.

\footnotetext{
${ }^{*}$ Em geral o colapso gravitacional realista forma um buraco negro sem superfície de bifurcação. No entanto supondo que o estado final possa ser descrito por um espaço-tempo com campo de Killing tal que o horizonte de Killing coincida com o horizonte de eventos então pode-se mostrar que existe uma extensão analítica de tal espaço-tempo que contém uma superfície de bifurcação [47,48]. Aqui então a superfície de Cauchy é referente não ao espaço-tempo físico mas sim a sua extensão analítica a um buraco negro eterno e a superfície é de Cauchy para espaço exterior ao buraco negro.

${ }^{+}$Perceba aqui o abuso da notação onde $\nabla \chi$ denota a rigor $\nabla_{a} \chi_{b}$.
} 


\subsection{O caso da Relatividade Geral}

O formalismo da carga de Noether permite derivar a primeira lei da dinâmica dos buracos negros de modo direto. Porém resta verificar que o formalismo reproduz os resultados da Relatividade Geral quando escolhemos a lagrangiana de Einstein-Hilbert. Nessa seção utilizaremos a notação de índices abstratos para sermos sucintos. A forma lagrangiana invariante por difeomorfismos é

$$
\mathbf{L}_{a b c d}=\frac{1}{16 \pi} R \epsilon_{a b c d}
$$

que tem como potencial simplético

$$
\boldsymbol{\Theta}_{a b c}=\frac{1}{16 \pi} \epsilon_{d a b c} g^{d e}\left(\nabla^{f} \delta g_{e f}-g^{f h} \nabla_{e} \delta g_{f h}\right),
$$

que consiste apenas do termo de superfície de Gibbons-Hawking-York [4], de modo que podemos calcular diretamente a corrente de Noether (note aqui que a forma simplética tem papel apenas de demonstrar que a variação da hamiltoniana é nula)

$$
\mathbf{J}_{a b c}=\frac{1}{8 \pi} \epsilon_{d a b c} \nabla_{e}\left(\nabla^{[e} \chi^{d]}\right),
$$

e por fim temos a carga de Noether

$$
\mathbf{Q}_{a b}=-\frac{1}{16 \pi} \epsilon_{a b c d} \nabla^{c} \chi^{d}
$$

que é fácil verificar [4] é igual a $\frac{\varkappa}{4 \pi} \varepsilon_{a b}$ sobre a superfície de bifurcação. Ainda mais no caso do campo vetorial ser uma translação temporal a energia canônica é

$$
M=-\frac{1}{8 \pi} \int_{\infty} \epsilon_{a b c d} \nabla^{c} t^{d}
$$

que corresponde a expressão para a massa de Komar [4]. De modo análogo o momento angular é

$$
J=\frac{1}{16 \pi} \int_{\infty} \epsilon_{a b c d} \nabla^{c} \varphi^{d},
$$

e portanto vemos que o formalismo da carga de Noether de fato é equivalente às demonstrações usuais da $1^{\mathrm{a}}$ lei. 


\section{Capítulo 7}

\section{Dinâmica Gravitacional e}

\section{Termodinâmica}

$\mathrm{N}^{\circ}$

o capítulo anterior vimos como a $1^{\text {a }}$ lei da dinâmica de buracos negros pode ser compreendida em função da carga de Noether. Essa abordagem sugere que a entropia pode ser entendida como um objeto geométrico definido localmente. Ainda mais a carga de Noether tem uma forma funcional diretamente dependente da lagrangiana, de modo que diferentes dinâmicas implicam, em princípio, em formas distintas para a entropia associada a buracos negros. A priori isso contrasta com o fato de que a segunda lei dinâmica, a saber que a área é uma função não-decrescente, pode ser obtida de argumentos puramente cinemáticos. Por outro lado qualquer função não-decrescente da área $f(A)$ terá $\Delta f \geq 0$ e portanto satisfaria a segunda lei da termodinâmica se tomada pela entropia. Desse modo fica claro que a forma funcional de $f$ será determinada pela primeira lei e assim sendo pela dinâmica.

Nesse capítulo iremos explorar a relação entre as equações de Einstein para a Relatividade Geral e a termodinâmica de um ponto de vista local seguindo a abordagem em [50]. No intuito de manter o foco na interpretação física o nível de rigor matemático nesse capítulo é notoriamente menor que ao longo do resto do texto. Iremos no entanto indicar os pontos onde os argumentos são meramentes formais, e indicamos, quando possível, onde o leitor interessado pode encontrar uma discussão mais detalhada. 


\subsection{O Horizonte Local de Rindler}

O formalismo da carga de Noether sugere que localmente devemos identificar a entropia com "áreas infinitesimais" a serem propriamente definidas. Postergando esse ponto é flagrante que um ponto delicado está em definir uma temperatura local. O plano geral consiste, seguindo [50], no seguinte argumento: dado que localmente qualquer variedade lorentziana pode ter sua métrica aproximada pela de Minkowski, então podemos igualmente aproximar a métrica pela de Rindlert Desse modo definiremos um horizonte local e uma temperatura através do efeito Unruh ${ }^{\dagger}[51]$.

Seja $(\mathcal{M}, \mathbf{g})$ uma variedade lorentziana qualquer e $p$ um ponto dessa variedade. Denotamos por $O$ a vizinhança normal convexa de $p$, isto é o aberto da variedade onde podemos introduzir coordenadas normais através da aplicação do mapa exponencial em uma dada base de $T_{p}$. A base apropriada para o espaço tangente será dada por uma tetrada nula complexa $\{\mathbf{k}, \boldsymbol{\ell}, \mathbf{m}, \overline{\mathbf{m}}\}$ tal que escolhemos $\theta=\sigma=\omega=0$ em $p$. Definimos então as coordenadas nulas normais em $O x^{\alpha}=(v, u, z, \bar{z})$ pela aplicação do mapa exponencial na base nula complexa escolhida como usual (note que ao contrário de uma base 1 +3 existe uma ambiguidade que não pode ser removida na normalização dos vetores nulos, aquela dada pelas rotações de Classe III, que no entanto corresponde a uma mudança de escala nas coordenadas que é irrelevante para os nossos propósitos).

Desejamos agora construir um horizonte local. Por motivos que ficarão claros adiante vamos escolher um ponto $p_{0} \in J^{-}(p)$ na geodésica formada por $\mathbf{k}$ que se encontre em uma coordenada arbitrária $v_{0}<0$. Definimos então o horizonte local de Rindler $H$ como a hiperfície nula dada por $u=0, v_{0} \leq v<0$ e identificamos a superfície $u=0, v=v_{0}$ como a superfície de bifurcação desse horizonte. Em analogia com o caso minkowskiano denotaremos as regiões de $O$ dadas por $v>v_{0}, u>0$ e $v>v_{0}, u<0$ como interior e exterior ao horizonte respectivamente.

Dado um horizonte local buscamos uma família de trajetórias às quais podemos associar uma temperatura, tendo em mente o efeito Unruh. Uma escolha óbvia é 
definir um vetor de Killing local como $\xi^{\alpha}=\varkappa\left(v-v_{o}\right) \delta_{v}^{\alpha}-u \delta_{u}^{\alpha}+\mathscr{O}\left(x^{2}\right)$, onde podemos escolher os coeficientes dos termos quadráticos de modo que a equação de Killing seja válida em segunda ordem ao redor do horizonte local de Rindler [52], ao passo que se omitirmos esses termos a equação de Killing só poderá valer em primeira ordem perto de $H$, e temos $\xi=\varkappa\left(v-v_{0}\right) \mathbf{k}$ em $H$. Resta saber em que medida a constante $\varkappa$ é arbitrária. Para avaliar isso considere que $\varkappa$ é uma escala de distância, que está relacionada com a magnitude da aceleração de uma trajetória específica do campo de Killing local no exterior do horizonte. Existem duas outras escalas de distância relevantes no problema, a relacionada a $\mathcal{R}=\max \left\{\left|R_{\mu v \lambda \rho}\right|\right\}$ na vizinhança normal e $L$ que é o comprimento coordenado da trajetória $\xi$. Tanto a curvatura quanto o tamanho finito da trajetória devem em geral ser relevantes para o espectro visto pelos observadores que seguem tal trajetória. Assim sendo considere uma constante $a$ que satisfaça $1 / a^{2}<<1 / \mathcal{R}$ e $a L>>1$, onde a primeira desigualdade denota que a escala de aceleração é muito maior que a introduzida pela curvatura, e a segunda que o comprimento da trajetória é muito maior que a escala de tamanho da vizinhança normal [53]. No caso em que $\varkappa \in(a,+\infty)$ é razoável supor que o efeito dominante será o da aceleração da trajetória e desse modo podemos associar uma temperatura a tais observadores de acordo com o efeito Unruh, de modo que as correções sejam sub-dominantes.

\subsection{As Equações de Einstein sob Nova Perspectiva}

De posse de um horizonte e de uma temperatura local é natural se perguntar se existe relação entre a dinâmica e uma relação termodinâmica considerando apenas a vizinhança normal convexa. Supondo a situação da Relatividade Geral a carga de Noether nos induz a uma relação de proporcionalidade entre a área e a entropia. Desse modo sendo uma superfície bi-dimensional espacial gerada pelos vetores $\{\mathbf{m}, \overline{\mathbf{m}}\}$, a variação da área entre $p_{0}$ e $p$ é dada por $\delta A=\int_{v_{0}}^{0} d v \int d^{2} \Sigma \theta$, onde $d^{2} \Sigma=\sqrt{\varsigma} d^{2} z$ e $\varsigma$ o determinante da métrica espacial da superfície. Com base na termodinâmica de buracos negros é natural supor que a variação de entropia associada é dada por $\delta S=\eta \delta A$, sendo $\eta$ uma 
constante positiva a ser determinada. A temperatura do sistema será dada pelo efeito Unruh, como motivada acima, isto é $T=\frac{\hbar \varkappa}{2 \pi}$.

Pretendendo utilizar uma relação termodinâmica como $\delta Q=T \delta S$ nos falta determinar o fluxo de calor. Vamos definir o fluxo de calor do exterior para o interior do horizonte local de Rindler, conforme observado pelos observadores em $\xi$, por $\delta Q=\int_{v_{0}}^{0} d v \int d^{2} \Sigma \mathrm{T}(\xi, \mathbf{k})$, ou seja como a variação da energia avaliada sobre os geradores do horizonte. Perceba que existem aqui duas arbitrariedades, a primeira é com relação a rotações de Classe III da base, e a segunda devido a $\varkappa$. A primeira é irrelevante pois introduz uma escala em $\mathbf{k}$ que aparece inversamente no parâmetro afim $v$ e a segunda desaparece na razão $\delta Q / T$ e sendo $\varkappa \neq 0$ de acordo com as hipóteses a expressão é bem definida. Para encontrar um vínculo entre o tensor energia-momento $T$ e a geometria da área vamos utilizar a primeira equação de Sachs (2.1.5):

$$
D \theta=-\frac{1}{2} \theta^{2}+2 \omega^{2}-2|\sigma|^{2}-\operatorname{Ric}(\mathbf{k}, \mathbf{k})
$$

Mas a expansão é dada por $\theta=D \ln \sqrt{\varsigma}$ de modo que $D \theta=(\sqrt{\varsigma})^{-1} D^{2} \sqrt{\varsigma}-\theta^{2}$, que utilizamos para reescrever a equação acima como

$$
\frac{1}{\sqrt{\varsigma}} D^{2} \sqrt{\varsigma}=+\frac{1}{2} \theta^{2}+2 \omega^{2}-2|\sigma|^{2}-\operatorname{Ric}(\mathbf{k}, \mathbf{k}) \text {. }
$$

Lembrando agora que escolhemos a tetrada nula complexa tal que em $p$ temos $\theta=\sigma=$ $\omega=0$ obtemos a expressão $(\mathrm{em} p)$

$$
\frac{1}{\sqrt{\varsigma}} D^{2} \sqrt{\varsigma}=-\operatorname{Ric}(\mathbf{k}, \mathbf{k})
$$

Vamos agora multiplicar ambos os lados de (7.2.3) por $\left(v-v_{0}\right)$ e integrar de 0 a $v_{0}$ sobre a área

$$
\int_{v_{0}}^{0} d v \int d^{2} \Sigma\left(v-v_{0}\right) \frac{1}{\sqrt{\varsigma}} D^{2} \sqrt{\varsigma}=-\int_{v_{0}}^{0} d v \int d^{2} \Sigma\left(v-v_{0}\right) \operatorname{Ric}(\mathbf{k}, \mathbf{k}),
$$

e agora desejamos avaliar o lado esquerdo da igualdade

$$
\int_{v_{0}}^{0} d v \int \sqrt{\varsigma} d^{2} z\left(v-v_{0}\right) \frac{1}{\sqrt{\varsigma}} D^{2} \sqrt{\varsigma}=\int d^{2} z \int_{v_{0}}^{0} d v\left(v-v_{0}\right) D^{2} \sqrt{\varsigma},
$$


que integrando por partes

$$
\begin{gathered}
\left.\int d^{2} z\left(v-v_{0}\right) D \sqrt{\varsigma}\right|_{v_{0}} ^{0}-\int d^{2} z \int_{v_{0}}^{0} d v D \sqrt{\varsigma}=\left.\int d^{2} z\left(v-v_{0}\right) D \sqrt{\varsigma}\right|_{v_{0}} ^{0}-\int_{v_{0}}^{0} d v \int d^{2} \Sigma \theta, \\
\int d^{2} \Sigma\left[\left(0-v_{0}\right) \theta(0)-\left(v_{0}-v_{0}\right) \theta\left(v_{0}\right)\right]-\int_{v_{0}}^{0} d v \int d^{2} \Sigma \theta
\end{gathered}
$$

de modo que trivialmente o primeiro termo é nulo e o segundo a variação da área, portanto resgatando (7.2.4)

$$
\delta A=\int_{v_{0}}^{0} d v \int d^{2} \Sigma\left(v-v_{0}\right) \operatorname{Ric}(\mathbf{k}, \mathbf{k}) .
$$

Agora com uma expressão para a variação da área podemos aplicar a relação $\delta Q=$ $T \delta S$

$$
\int_{v_{0}}^{0} d v \int d^{2} \Sigma \mathbf{T}(\xi, \mathbf{k})=\frac{\hbar \varkappa \eta}{2 \pi} \int_{v_{0}}^{0} d v \int d^{2} \Sigma\left(v-v_{0}\right) \operatorname{Ric}(\mathbf{k}, \mathbf{k})
$$

de modo que

$$
\int_{v_{0}}^{0} d v \int d^{2} \sum \varkappa\left(v-v_{0}\right)\left[\mathrm{T}(\mathbf{k}, \mathbf{k})-\frac{\hbar \eta}{2 \pi} \operatorname{Ric}(\mathbf{k}, \mathbf{k})\right]=0 .
$$

Note no entanto que a integral sobre $\Sigma$ é arbitrária, o que implica

$$
\mathrm{T}(\mathbf{k}, \mathbf{k})-\frac{\hbar \eta}{2 \pi} \operatorname{Ric}(\mathbf{k}, \mathbf{k})=0
$$

e sendo a escolha do vetor $\mathbf{k}$ arbitrária (entre os vetores nulos em $p$ )

$$
\operatorname{Ric}=\frac{2 \pi}{\hbar \eta}[\mathbf{T}-f \mathbf{g}]
$$

onde $f$ é um escalar arbitrário. Agora impondo o vínculo do gradiente nulo do tensor energia-momento, e denotando $T=$ trace $T$,

$$
\text { Ric }=\frac{2 \pi}{\hbar \eta}\left[\mathrm{T}-\frac{1}{2} \mathbf{g} T\right]
$$

Exigindo que a expansão para campos fracos seja equivalente a teoria newtoniana determinamos $\eta=(4 \hbar G)^{-1}$. 


\subsection{Da Termodinâmica para a Gravitação(?)}

Cabe agora nos perguntarmos se o argumento presente na seção anterior consiste de uma derivação das equações de Einstein a partir de uma equação de estado termodinâmica como pretende [50]. Estando a álgebra correta e supondo que a definição de horizonte local de Rindler e sua temperatura associada correspondam de fato ao entendimento do efeito Unruh do ponto de vista loca| لntão seguem de uma definição apropriada de fluxo de calor as equações de Einstein.

É importante ainda mais verificar o contexto físico dessa linha de raciocínio. Segundo Jacobson [50] sendo as equações da dinâmica gravitacional uma consequência da termodinâmica então é sugestivo pensar que seja o que for a gravitação quântica é possível que a teoria clássica surja como um efeito coletivo dos graus de liberdade microscópicos. Assim não seria apropriado aplicar o procedimento de quantização da ação como para as outras interações, do mesmo modo que é inapropriado tentar quantizar diretamente a equação de movimento para uma onda sonora, no sentido em que não obtemos uma descrição fundamental do sistema mas sim uma teoria efetiva de um efeito de muitos corpos, como os fônons. Com isso tal argumentação levanta a questão de que existem duas rotas da mecânica quântica para a clássica, uma na qual $\hbar \rightarrow 0$ e apenas as trajetórias que minimizam a ação se tornam relevantes e o caminho via mecânica estatística. Nesse ponto é notório que o limite apropriado para a qual as estatísticas quânticas, a saber Bose-Einstein e Fermi-Dirac, tendem a uma estatística clássica, Maxwell-Boltzmann, é $T \rightarrow \infty$, isto é para altas temperaturas, que faz parte das suposições da seção 7.1. Veja que tal hipótese foi feita unicamente para que o efeito Unruh seja dominante sobre a curvatura e o tempo próprio finito disponível aos observadores, e assim não parece diretamente dependente de tentarmos relacionar a termodinâmica com as equações de Einstein.

No entanto é premente fazer algumas críticas. Supondo que o efeito Unruh possa ser estabelecido pela teoria quântica de campos em espaços curvos de acordo com a

*Algo que ainda não foi bem estabelecido até o limite do conhecimento deste autor. 
linha apresentada é evidente que o ponto mais delicado da argumentação reside em tomar $\theta=\sigma=\omega=0$. Note que ao passo que o vetor $\mathbf{k}$ é arbitrário o mesmo não se verifica para a tetrada complexa nula escolhida, dado que é ela quem define os escalares ópticos. Isso implica que as seções espaciais do horizonte de eventos local não podem ser escolhidas arbitrariamente. Ainda mais, a validade das integrações depende dos escalares ópticos sofrerem correções no máximo de ordem quadrática em $x^{\alpha}$, o que não é óbvio corresponder ao caso geral. Segundo Jacobson tal escolha de base se reflete em exigir consistência com a termodinâmica de equilíbrio. Caso desejássemos incluir, por exemplo, os termos de cisalhamento deveríamos incluir uma contribuição da entropia fora de equilíbrio devido a viscosidade. Para mostrar que esse argumento não tem fundamentação física vamos considerar um exemplo simples.

O fluido ideal é definido como aquele sem viscosidade, e como essa é definida como um coeficiente relacionado ao cisalhamento do campo de velocidades a suposição de fluido ideal implica inexoravelmente que o mesmo seja nulo. Podemos questionar então se dentro da mecânica de fluidos ideais nos restringimos apenas a fenômenos termodinâmicos em equilíbrio. Fica claro que isso não procede considerando ondas de choque. De acordo com [54] quando há formação de ondas de choque a densidade de entropia sofre sempre um aumento de modo que não podemos aplicar equações de termodinâmica de equilíbrio. Conversamente considere o gás perfeito, isto é o fluido que obedece a equação de estado de Clapeyron. O gás perfeito é tipicamente turbulento e ainda assim descrito pela termodinâmica de equilíbrio usual. Portanto vemos que a questão do equilíbrio termodinâmico não é estritamente correlacionada com o cisalhamento e portanto não podemos resgatar a derivação apelando para uma instância superior do formalismo termodinâmico.

Note que embora a argumentação seja inspirada na derivação física da $1^{\mathrm{a}}$ lei da dinâmica de buraco negros encontrada em [35] ela difere em um ponto essencial. Em [35] o ponto de partida da construção é o ponto onde se encontra a superfície de bifurcação e a integração é feita até o limite $v \rightarrow \infty$. Assim o ponto $p$ central para a construção de Jacobson corresponde no caso do buraco negro a um ponto pertencente 
ao espaço não-físico $(\widetilde{\mathcal{M}}, \tilde{\mathbf{g}})$, em particular pertence a $\mathscr{I}^{+}$. Note que tal construção no caso local é essencial para que o primeiro termo em (7.2.7) se anule, e portanto tal discrepância é parte integrante da argumentação.

Por fim gostaríamos de salientar que revertendo a lógica de Jacobson podemos a partir das equações de Einstein demonstrar facilmente a $1^{a}$ lei da mecânica dos buracos negros seguindo essencialmente ordem inversa da argumentação supondo o caso do buraco negro como em [35]. No caso do buraco negro note que as escolhas de $\theta=\sigma=0$ são impostas diretamente pelo buraco negro estacionário, a primeira pela coincidência do horizonte aparente com o horizonte de eventos e a segunda pelo fato de que $\mathbf{k}$ é um vetor principal nulo de um espaço-tempo algebricamente especial. Infelizmente não podemos usar a dinâmica dos buracos negros para deduzir as equações de Einstein justamente porque o vetor $\mathbf{k}$ não é arbitrário e nem a superfície de integração. 


\section{Capítulo 8}

\section{Conclusões e Perspectivas}

ENDo esse um trabalho dedicado a explorar as questões termodinâmicas presentes
na Relatividade Geral, em especial em buracos negros, o leitor pode ter notado que em momento algum ofereci uma justificativa acerca da relevância de tais questões. Uma postura plausível, e em certa parte adotada aqui, seria análoga a de Gautier l'art pour l'art com o que quero dizer que os buracos negros são objetos extremamente interessantes, como evidenciado pelas discussões acerca das propriedades globais e locais dos espaços-tempo discutidos nos capítulos 2, 3 e 4, de modo que o seu estudo é tão revelador das características que fazem da Relatividade Geral ímpar que seu estudo se justifica em si mesmo. As propriedades termodinâmicas são tão surpreendentes que apenas corroboram tal postura.

Uma outra possibilidade, certamente a que prevalece, é tomar a postura de Boltzmann $\bigsqcup^{\dagger}$ de que a entropia é atrelada ao número de configurações microscópicas às quais correspondem uma mesma configuração macroscópica e que sua existência é indicadora de que um buraco negro, e a Relatividade Geral, possui graus de liberdade microscópicos desconhecidos no presente. De fato todas as teorias que se propõem ao papel de gravitação quântica tem o hábito de listar entre seus sucessos a capacidade de derivar a proporcionalidade da entropia com a área dos buracos negros. Assim a importância da termodinâmica no contexto da Relatividade Geral é fornecer uma 
janela para a gravitação quântica. Uma alternativa a quantização da gravidade reside nas abordagens chamadas "gravitação emergente", iniciadas por Sakharov网[55], onde ao invés de quantizar a gravitação supomos que a Relatividade Geral emerge como um efeito de muitos corpos em uma teoria do espaço-tempo subjacente. Tal abordagem tem recentemente atraído mais atenção após a publicação da "derivação" das equações de Einstein por Jacobson [50] que discutimos no capítulo 7. Embora a proposta de Jacobson não possa ser tomada como uma demonstração formal, ainda mais frente ao padrão estabelecido pelos resultados clássicos, é digno de nota que alguns de seus aspectos, como a necessidade de uma aceleração alta para o efeito Unruh, parecem ser remanescentes de um limite clássico para sistemas quânticos. Note que no entanto tal sucesso deve ser balanceado com o preço a ser pago com escolhas arbitrárias sobre a tetrada sem as quais a derivação não é possível, ou então caso se abra mão, por exemplo, da expansão nula se é levado a identificar um termo associado à energia gravitacional quase-local [56], algo que não pertence ao cânones da Relatividade Geral. Desse modo, e considerando o progresso que a Relatividade fez atendo-se a resultados rigorosos, nos parece que a abordagem ilustrada no capítulo 7 deve ser vista no máximo como uma interpretação das equações de Einstein e não uma derivação.

Tipicamente se argumenta que a razão do aparente sucesso de tal abordagem reside no fato de que a termodinâmica é insensível aos graus de liberdade microscópicos e portanto mesmo partindo de um ponto semi-clássico é possível se deduzir as equações de Einstein [52]. Tal postura é apenas parcialmente verdadeira, pois se é verdade que a $1^{\mathrm{a}} \mathrm{e}$ $2^{\mathrm{a}}$ lei da termodinâmica, junto com a lei zero, parecem ser de caráter geral para sistemas em equilíbrio não procede que essa seja a estória completa. Como enfatizamos na seção 5.3 a terceira lei da termodinâmica é dependente diretamente da não-degenerescência do estado fundamental do sistema. Usualmente a violação no enunciado de Planck é tomado por ponto passivo considerando-se o caso de Kerr extremo, porém não é claro até que ponto deveríamos considerar a solução de Kerr extrema como um sistema termodinâmico pleno. Em particular nota-se a dependência do horizonte de Killing ${ }^{*}$ Andrei Dmitrievich Sakharov (1921-1989) 
ser bifurcado para demonstrar a unicidade da solução. Além disso a abordagem da carga de Noether discutida no capítulo 6 sugere fortemente atribuir entropia nula ao caso extremo o que põe em cheque sua natureza termodinâmica. Outra assinatura da dinâmica microscópica está, em princípio, na transição de Davies, por exemplo se pudermos ser capazes de identificar a qual simetria se relaciona a transição e/ou seu parâmetro de ordem. Sabemos, por exemplo, que na transição de fase quaisquer que sejam os graus de liberdade estes não podem ser tratados pela abordagem de campo médio. Nos parece que tanto a terceira lei da termodinâmica de buracos negros como a transição de Davies são merecedoras de maiores investigações dado seu potencial para entendermos parte da teoria quântica da gravidade quando comparados com o seu status atual bastante insatisfatório em relação às demais leis.

Se o comportamento termodinâmico dos buracos negros oferece uma janela para o entendimento da Relatividade Geral o converso também pode ser dito. A Relatividade oferece um panorama possivelmente único para explorar a termodinâmica em um contexto onde temos técnicas para proceder rigorosamente, somos obrigados a formular a teoria na ausência de poder impor vínculos como paredes para os sistemas e mais importante de tudo é um caso singular onde o formalismo termodinâmico tem incorporado naturalmente a noção de tempo através da evolução nas superfícies de Cauchy. A segunda lei da mecânica dos buracos negros é aqui particularmente destacável pois se interpretada termodinamicamente podemos de fato assegurar a relação entre o crescimento da entropia e a evolução temporal.

Assim a termodinâmica de buracos negros, bem como suas possíveis generalizações, fornece um laboratório tanto para a exploração das questões tradicionais da Relatividade Geral, a eventual teoria de gravitação quântica e para os fundamentos da termodinâmica e mecânica estatística. 


\section{Referências}

[1] PENROSE, R. Structure of space-time. In: DEWITT-MORETTE, C.; WHEELER, J. A. (Ed.). Battelle rencontres. New York: W. A. Benjamin, 1967. cap. 10.

[2] PETROV, A. Z. Einstein spaces. Oxford: Pergamon Press, 1969.

[3] SACHS, R. K. Gravitational waves in general relativity. VI. the outgoing radiation condition. Proceedings of the Royal Society of London A, v. 264, n. 1318, p. 309 - 338, 1961.

[4] WALD, R. M. General relativity. Chicago: The University of Chicago Press, 1984.

[5] O'NEILL, B. The geometry of Kerr black holes. Wellesley: A. K. Peters, 1995.

[6] SZEKERES, P. The gravitational compass. Journal of Mathematical Physics, v. 6, n. 9, p. 1387 - 1391, 1965.

[7] D'INVERNO, R. Introducing Einstein's relativity. Oxford: Oxford University Press, 1992.

[8] SACHS, R. K. Gravitational waves in general relativity. VIII. waves in asymptoptically flat space-time. Proceedings of the Royal Society of London A, v. 270, n. 1340, p. $103-126,1962$.

[9] RYDER, L. Introduction to general relativity. Cambridge: Cambridge University Press, 2009.

[10] NEWMAN, E.; PENROSE, R. An aproach to gravitational radiation by a method of spin coefficients. Journal of Mathematical Physics, v. 3, n. 3, p. 566 - 578, 1962. 
[11] GOLDBERG, J. N.; SACHS, R. K. A theorem on Petrov types. Acta Physica Polonica, v. 22, n. 13, p. $13-23,1962$.

[12] CHOQUET-BRUHAT, Y.; DEWITT-MORETTE, C.; DILLARD-BLEICK, M. Analisys, manifolds, and physics. Amsterdam: North-Holland, 1982.

[13] BONDI, H.; VAN DER BURG, G. J.; METZNER, A. W. K. Gravitational waves in general relativity. VII. waves from axi-symmetric isolated systems. Proceedings of the Royal Society of London A, v. 269, n. 1336, p. 21 - 52, 1962.

[14] CHANDRASEKHAR, S. The maximum mass of ideal white dwarfs. Astrophysical Journal, v. 74, p. 81 - 82, 1931.

[15] OPPENHEIMER, J. R.; VOLKOFF, G. M. On massive neutron cores. Physical Review, v. 55, n. 4, p. $374-381,1939$.

[16] OPPENHEIMER, J. R.; SNYDER, H. On continued gravitational contraction. Physical Review, v. 56, n. 5, p. 455 - 459, 1939.

[17] HAWKING, S. W.; ELLIS, G. F. R. The large scale structure of space-time. Cambridge: Cambridge University Press, 1973. (Cambridge monographs on mathematical physics).

[18] JEANS, J. H. The stability of a spherical nebula. Philosophical Transactions of the Royal Society of London A, v. 199, p. 1 - 53, 1902.

[19] CLARKE, C. J. S. A condition for forming trapped surfaces. Classical and Quantum Gravity, v. 5, n. 7, p. 1029 - 1032, 1998.

[20] PENROSE, R. The question of cosmic censorship. In: WALD, R. M. (Ed.). Black holes and relativistic stars. Chicago: The University of Chicago Press, 1994. cap. 5.

[21] CHRÚSCIEL, P.; WALD, R. M. On the topology of stationary black holes. Classical and Quantum Gravity, v. 11, n. 12, p. L147 - L52, 1994.

[22] GIULINI, D. Local version o the area theorem (on a question by G. $\mathrm{t}^{\prime}$ Hooft). In: HEHL, F. W.; KIEFER, C.; METZLER, R. J. K. (Ed.). Black holes - theory and observation. Berlin: Springer-Verlag, 1998. cap. 9. 
[23] BARDEEN, J. M.; CARTER, B.; HAWKING, S. W. The four laws of black hole mechanics. Communications in Mathematical Physics, v. 31, n. 2, p. 161 - 170, 1974.

[24] KERR, R. P. The Kerr and Kerr-Schild metrics. In: WILTSHIRE, D. L.; VISSER, M.; SCOTT, S. M. (Ed.). The Kerr spacetime - rotating black holes in general relativity. Cambridge: Cambridge University Press, 2009. cap. 2.

[25] KERR, R. P. Gravitational field of a spínning mass as an example of algebraically special metrics. Physical Review Letters, v. 11, n. 5, p. 237 - 238, 1963.

[26] PENROSE, R. Gravitational collapse: the role of general relativity. Rivista del Nuovo Cimento, v. 1, n. 1, p. 252 - 276, 1969.

[27] CHRISTODOULOU, D. Reversible and irreversible transformations in black-hole physics. Physical Review Letter, v. 25, n. 22, p. 1596 - 1597, 1970.

[28] CARTER, B. The general theory of mechanical, electromagnetical and thermodynamic properties of black holes. In: HAWKING, S. W.; ISRAEL, W. (Ed.). General relativity: an Einstein centenary survey. Cambridge: Cambridge University Press, 1979. cap. 6.

[29] NEUGEBAUER, G.; HENNIG, J. Non-existence of stationary two-black-hole configurations. General Relativity and Gravitation, v. 41, n. 9, p. 2113 - 2130, 2009.

[30] ROBINSON, D. C. Four decades of black holes uniqueness theorems. In: WILTSHIRE, D. L.; VISSER, M.; SCOTT, S. M. (Ed.). The Kerr spacetime - rotating black holes in general relativity. Cambridge: Cambridge University Press, 2009. cap. 5.

[31] BEKENSTEIN, J. D. Black holes and entropy. Physical Review D, v. 7, n. 8, p. 2333 2346, 1973.

[32] HAWKING, S. W. Black hole explosions? Nature, v. 248, n. 5443, p. 30 - 31, 1974.

[33] HAWKING, S. W. Particle creation by black holes. Communications in Mathematical Physics, v. 43, n. 3, p. 199 - 220, 1975.

[34] POISSON, E. A relativist's toolkit: the mathematics of black-hole mechanics. Cambridge: Cambridge University Press, 2004. 
[35] WALD, R. M. Quantum field theory in curved spacetime and black hole thermodynamics. Chicago: The University of Chicago Press, 1994. (Chicago lectures in physics).

[36] REISS, H. Methods of Thermodynamics. Mineola: Dover, 1965.

[37] FARRUGIA, C. J.; HAJICEK, P. The third law of black hole mechanics: a counterexample. Communications in Mathematical Physics, v. 68, n. 3, p. 291 - 300, 1979.

[38] ISRAEL, W. Third-law of black-hole dynamics: a formulation and proof. Physical Review Letters, v. 57, n. 4, p. 397 - 399, 1986.

[39] DAVIES, P. C. W. The thermodynamics theory of black holes. Proceedings of the Royal Society of London A, v. 353, n. 1675, p. 499 - 521, 1977.

[40] MATSAS, G. E. A.; RICHARTZ, M.; SAA, A.; DA SILVA, A. R. R.; VANZELLA, D. A. T. Can quantum mechanics fool the cosmic censor? Physical Review D, v. 79, n. 10, p. 101502(R) , 2009.

[41] RÁCZ, I. Does the third law of black hole thermodynamics really have a serious failue? Classical and Quantum Gravity, v. 17, n. 20, p. 4353 - 4356, 2000.

[42] KADANOFF, L. P. Statistical physics - statics, dynamics and renormalization. Singapore: World Scientific, 2000.

[43] APOSTOL, T. M. Introduction to analytic number theory. New York: Springer-Verlag, 1976.

[44] WALD, R. M. Black hole entropy is the Noether charge. Physical Review D, v. 48, n. 8, p. R3427 - R3431, 1993.

[45] LEE, J.; WALD, R. M. Local symmetries and constraints. Journal of Mathematical Physics, v. 31, n. 3, p. 725 - 744, 1990.

[46] IYER, V.; WALD, R. M. Some properties of the Noether charge and a proposal for a dynamical black hole entropy. Physical Review D, v. 50, n. 2, p. 846 - 864, 1994.

[47] RÁCZ, I.; WALD, R. M. Extensions of spacetimes with Killing horizons. Classical and Quantum Gravity, v. 9, n. 12, p. 2643 - 2656, 1992. 
[48] RÁCZ, I.; WALD, R. M. Global extensions of spacetimes describing asymptotic states of black holes. Classical and Quantum Gravity, v. 13, n. 3, p. 539 - 552, 1996.

[49] HAWKING, S. W.; HOROWITZ, G. T. Entropy, area and black hole pairs. Physical Review D, v. 51, n. 8, p. 4302 - 4314, 1995.

[50] JACOBSON, T. Thermodynamics of spacetime: the Einstein equation of state. Physical Review Letters, v. 75, n. 7, p. 1260 - 1263, 1995.

[51] CRISPINO, L. C. B.; HIGUSHI, A.; MATSAS, G. E. A. The Unruh effect and its applications. Reviews of Modern Physics, v. 80, n. 3, p. 787 - 838, 2008.

[52] GUEDENS, R.; JACOBSON, T.; SARKAR, S. Horizon entropy and higher curvature equation of state. Physical Review D, v. 85, n. 6, p. 064017 , 2012.

[53] MARTINETTI, P.; ROVELLI,C. Diamond's temperature: Unruh effect for bounded trajectories and thermal time hypothesis. Classical and Quantum Gravity, v. 20, n. 22, p. 4919 - 4931, 2003.

[54] CHORIN, A. J.; MARSDEN, J. E. A mathematical introduction to fluid mechanics. New York: Springer, 1997.

[55] SAKHAROV, A. D. Vacuum quantum fluctuations in curved space and the theory of gravitation. General Relativity and Gravitation, v. 32, n. 2, p. 365 - 367, 2000.

[56] KOTHAWALA, D. Thermodynamics structure of Einstein tensor. Physical Review D, v. 83, n. 2, p. $024026,2011$.

[57] O'NEILL, B. Semi-riemannian geometry: with applications to general relativity. San Diego: Academic Press, 1983.

[58] STEWART, J. Advanced general relativity. Cambridge: Cambridge University Press, 1991.

[59] GEROCH, R.; HOROWITZ, G. T. Global structure of spacetimes. In: HAWKING, S. W.; ISRAEL, W. (Ed.). General relativity: an Einstein centenary survey. Cambridge: Cambridge University Press, 1979. cap. 5.

[60] PENROSE, R. Gravitational collapse and space-time singularities. Physical Review Letters, v. 14, n. 3, p. 57 - 59, 1965. 


\section{Apêndice A}

\section{Formas de Cartan e Tetradas Nulas}

1 lar objetos geométricos. A metodologia de Cartan ${ }^{\mid}$para a geometria diferencial no entanto fornece um modo alternativo de caracterizar a geometria de uma variedade, usando a estrutura de formas diferenciais [57]. Iremos aqui meramente resumir os principais pontos desse método que serão necessários neste ensaio. Além disso aproveitamos o espaço para explicitar as equações estruturais, entre outras, para o formalismo de Newman-Penrose.

\section{A.1 O Método do Referencial Móvel}

Considere uma base $\left\{\mathbf{E}_{\mu}\right\}$ em uma variedade semi-riemanniana $(\mathcal{M}, \mathbf{g})$ tal que $\left(\mathbf{E}_{\mu} \mid \mathbf{E}_{v}\right)=$ $\eta_{\mu v}$, onde $\eta_{\mu v}$ são as componentes da métrica de Minkowski na base $\left\{\mathbf{E}_{\mu}\right\}$. Então $\left\{\mathbf{E}_{\mu}\right\}$ é uma tetrada ou um referencial móvel. A cotetrada $\left\{\omega^{\mu}\right\}$ é formada pelas 1-formas diferenciais dadas por $\omega^{\mu}\left(\mathbf{E}_{v}\right)=\delta^{\mu}{ }_{v}$. Note que isso implica que podemos usar a métrica de Minkowski para levantar e rebaixar índices das tetradas do modo usual.

Definição A.1.1 As formas de conexão de uma tetrada $\left\{\mathbf{E}_{\mu}\right\}$ são as 1-formas diferenciais $\boldsymbol{\omega}^{\mu}{ }_{\nu}$ tais que para qualquer vetor $\mathbf{v}$ temos $\omega^{\mu}{ }_{v}(\mathbf{v})=\omega^{\mu}\left(\nabla_{\mathbf{v}} \mathbf{E}_{v}\right)$ onde $\nabla$ é uma conexão de Levi-Civita. *Élie Joseph Cartan (1869-1951). 
Usando a fórmula da dualidade para tetradas, a saber $\mathbf{X}=\sum_{\mu} \omega^{\mu}(\mathbf{X}) \mathbf{E}_{\mu}$ que decorre da cotetrada para qualquer $\mathbf{X} \in T_{p}$, temos que $\nabla_{\mathbf{v}} \mathbf{E}_{v}=\sum_{\mu} \omega^{\mu}{ }_{v}(\mathbf{v}) \mathbf{E}_{\mu}$. Portanto as formas de conexão atuando em $\mathbf{v}$ refletem a mudança em um vetor da base ao longo de uma direção v. Em especial as componentes $\omega^{\mu}{ }_{v}\left(\mathbf{E}_{\lambda}\right)=\omega_{\lambda}{ }^{\mu}{ }_{v}$ são chamados coeficientes de rotação de Ricci.

Lema A.1.2 Para as formas de conexão temos $\omega^{\mu}{ }_{v}=-\boldsymbol{\omega}^{v}{ }_{\mu}$ se $\mu, v>0$ e $\boldsymbol{\omega}_{v}^{0}=\boldsymbol{\omega}_{0}^{v}$.

Demonstração Dado que $\left(\mathbf{E}_{\mu} \mid \mathbf{E}_{v}\right)=\eta_{\mu v}$ é constante temos $\left(\nabla_{\mathbf{v}} \mathbf{E}_{\mu} \mid \mathbf{E}_{v}\right)+\left(\mathbf{E}_{\mu} \mid \nabla_{\mathbf{v}} \mathbf{E}_{v}\right)=0$ usando a compatibilidade com a métrica $\nabla_{\mathbf{v}} \mathbf{g}=0$. Usando $\nabla_{\mathbf{v}} \mathbf{E}_{\mu}=\sum_{\sigma} \omega_{\mu}^{\sigma}(\mathbf{v}) \mathbf{E}_{\sigma}$ temos $\sum_{\sigma} \omega^{\sigma}{ }_{\mu} \eta_{\sigma v}+\sum_{\sigma} \omega^{\sigma}{ }_{v} \eta_{\sigma v}=0$ do qual segue $\omega_{v \mu}+\omega_{\mu v}=0$ completando a demonstração.

Note que a antissimetria das formas de conexão implica que existem apenas 24 coeficientes de rotação de Ricci independentes. Vamos terminar esta seção com dois lemas que formam o cerne do método do referencial móvel, as demonstrações de ambos podem ser vistas em [5].

Lema A.1.3 (Primeira Equação Estrutural) Se $\nabla$ é uma conexão de torsão nula então

$$
d \omega^{v}=\sum_{\mu} \omega^{\mu} \wedge \omega_{\mu}^{v}
$$

Lema A.1.4 (Segunda Equação Estrutural) Sejam X, Y vetores arbitrários e $R$ o tensor de curvatura de Riemman. Definimos a 2-forma de curvatura por $\sum_{\mu} \mathbf{\Omega}_{\nu}^{\mu}(\mathbf{X}, \mathbf{Y}) \mathbf{E}_{\mu}=R_{\mathbf{X Y}}\left(\mathbf{E}_{v}\right)$. A forma de curvatura obedece

$$
\boldsymbol{\Omega}_{v}^{\mu}=d \boldsymbol{\omega}_{v}^{\mu}+\sum_{\sigma} \omega_{\sigma}^{\mu} \wedge \omega_{v}^{\sigma}
$$

\section{A.2 O Referencial Nulo}

Um referencial nulo, ou tetrada nula, consiste de uma base $\{\mathbf{k}, \boldsymbol{\ell}, \mathbf{m}, \overline{\mathbf{m}}\}$ tal que $(\mathbf{k} \mid \boldsymbol{\ell})=$ $-(\mathbf{m} \mid \overline{\mathbf{m}})=-1$ e todos os outros produtos internos nulos. Denotamos as derivadas em cada direção por $D=\nabla_{\mathbf{k}}, \Delta=\nabla_{\ell}, \delta=\nabla_{\mathbf{m}}$ e $\bar{\delta}=\nabla_{\overline{\mathbf{m}}}$. Os coeficientes de rotação de Ricci 
nesta base (coeficientes de spin) podem ser expressos através de 12 escalares complexos que definimos:

$$
\begin{array}{ccc}
\kappa=-(D \mathbf{k} \mid \mathbf{m}) & \nu=(\Delta \boldsymbol{\ell} \mid \overline{\mathbf{m}}) & \varepsilon=\frac{1}{2}[-(D \mathbf{k} \mid \boldsymbol{\ell})+(D \mathbf{m} \mid \overline{\mathbf{m}})] \\
\rho=-(\bar{\delta} \mathbf{k} \mid \mathbf{m}) & \mu=(\delta \boldsymbol{\ell} \mid \overline{\mathbf{m}}) & \gamma=\frac{1}{2}[+(\Delta \boldsymbol{\ell} \mid \mathbf{k})-(\Delta \overline{\mathbf{m}} \mid \mathbf{m})] \\
\sigma=-(\delta \mathbf{k} \mid \mathbf{m}) & \lambda=(\bar{\delta} \boldsymbol{\ell} \mid \overline{\mathbf{m}}) & \beta=\frac{1}{2}[-(\delta \mathbf{k} \mid \boldsymbol{\ell})+(\delta \mathbf{m} \mid \overline{\mathbf{m}})] \\
\tau=-(\Delta \mathbf{k} \mid \mathbf{m}) & \pi=(D \boldsymbol{\ell} \mid \overline{\mathbf{m}}) & \alpha=\frac{1}{2}[+(\bar{\delta} \boldsymbol{\ell} \mid \mathbf{k})-(\bar{\delta} \overline{\mathbf{m}} \mid \mathbf{m})] .
\end{array}
$$

A definição dos escalares implica que a derivada dos vetores da base pode ser representada pelas seguintes equações (mais as complexas-conjugadas):

$$
\begin{aligned}
& D \mathbf{k}=(\varepsilon+\bar{\varepsilon}) \mathbf{k}-\bar{\kappa} \mathbf{m}-\kappa \overline{\mathbf{m}} \\
& \Delta \mathbf{k}=(\gamma+\bar{\gamma}) \mathbf{k}-\bar{\tau} \mathbf{m}-\tau \overline{\mathbf{m}} \\
& \delta \mathbf{k}=(\beta+\bar{\alpha}) \mathbf{k}-\bar{\rho} \mathbf{m}-\sigma \overline{\mathbf{m}} \\
& D \boldsymbol{\ell}=-(\varepsilon+\bar{\varepsilon}) \mathbf{k}+\tau \mathbf{m}+\overline{\tau \mathbf{m}} \\
& \Delta \boldsymbol{\ell}=-(\gamma+\bar{\gamma}) \mathbf{k}+\nu \mathbf{m}+\overline{\nu \mathbf{m}} \\
& \delta \boldsymbol{\ell}=-(\beta+\bar{\alpha}) \mathbf{k}+\mu \mathbf{m}+\bar{\lambda} \overline{\mathbf{m}} \\
& D \mathbf{m}=\bar{\tau} \mathbf{k}-\kappa \boldsymbol{\ell}+(\varepsilon-\bar{\varepsilon}) \mathbf{m} \\
& \Delta \mathbf{m}=\bar{v} \mathbf{k}-\tau \boldsymbol{\ell}+(\gamma-\bar{\gamma}) \mathbf{m} \\
& \delta \mathbf{m}=\bar{\lambda} \mathbf{k}-\sigma \boldsymbol{\ell}+(\beta-\bar{\alpha}) \mathbf{m} \\
& \delta \overline{\mathbf{m}}=\mu \mathbf{k}-\bar{\rho} \boldsymbol{\ell}+(\bar{\alpha}-\beta) \overline{\mathbf{m}} .
\end{aligned}
$$

Os comutadores dos vetores da base são (aplicados a uma função escalar):

$$
\begin{aligned}
{[D, \Delta] } & =-(\gamma+\bar{\gamma}) D-(\varepsilon+\bar{\varepsilon}) \Delta+(\pi+\bar{\tau}) \delta+(\bar{\pi}+\tau) \bar{\delta} \\
{[D, \delta] } & =(\bar{\pi}-\bar{\alpha}-\beta) D-\kappa \Delta+(\varepsilon-\bar{\varepsilon}+\bar{\rho}) \delta+\sigma \bar{\delta} \\
{[\Delta, \delta] } & =\bar{v} D+(\bar{\alpha}+\beta-\tau) \Delta+(\gamma-\bar{\gamma}-\mu) \delta-\overline{\lambda \delta} \\
{[\delta, \bar{\delta}] } & =(\mu-\bar{\mu}) D+(\rho-\bar{\rho}) \Delta+(\bar{\beta}-\alpha) \delta+(\bar{\alpha}-\beta) \bar{\delta}
\end{aligned}
$$


As componentes (complexas) do tensor de curvatura de Weyl são definidas por:

$$
\begin{aligned}
& \Psi_{0}=\mathrm{C}(\mathbf{k}, \mathbf{m}, \mathbf{k}, \mathbf{m}) \\
& \Psi_{1}=\mathrm{C}(\mathbf{k}, \boldsymbol{\ell}, \mathbf{k}, \mathbf{m}) \\
& \Psi_{2}=\mathrm{C}(\mathbf{k}, \mathbf{m}, \overline{\mathbf{m}}, \boldsymbol{\ell}) \\
& \Psi_{3}=\mathrm{C}(\mathbf{k}, \boldsymbol{\ell}, \overline{\mathbf{m}}, \boldsymbol{\ell}) \\
& \Psi_{4}=\mathrm{C}(\boldsymbol{\ell}, \overline{\mathbf{m}}, \boldsymbol{\ell}, \overline{\mathbf{m}}) .
\end{aligned}
$$

As componentes do tensor de Ricci e o escalar de curvatura são definidos por:

$$
\begin{aligned}
\Phi_{00} & =\frac{1}{2} \operatorname{Ric}(\mathbf{k}, \mathbf{k}) \\
\Phi_{01} & =\frac{1}{2} \operatorname{Ric}(\mathbf{k}, \mathbf{m})=\bar{\Phi}_{10} \\
\Phi_{02} & =\frac{1}{2} \operatorname{Ric}(\mathbf{m}, \mathbf{m})=\bar{\Phi}_{20} \\
\Phi_{22} & =\frac{1}{2} \operatorname{Ric}(\boldsymbol{\ell}, \boldsymbol{\ell}) \\
\Phi_{12} & =\frac{1}{2} \operatorname{Ric}(\boldsymbol{\ell}, \mathbf{m})=\bar{\Phi}_{21} \\
\Phi_{11} & =\frac{1}{4}[\operatorname{Ric}(\mathbf{k}, \boldsymbol{\ell})+\operatorname{Ric}(\mathbf{m}, \overline{\mathbf{m}})] \\
\Lambda & =\frac{1}{24} R=\frac{1}{12}[-\operatorname{Ric}(\mathbf{k}, \boldsymbol{\ell})+\operatorname{Ric}(\mathbf{m}, \overline{\mathbf{m}})] .
\end{aligned}
$$


As componentes da curvatura são encontradas via a segunda equação estrutural produzindo as identidades de Ricci:

$$
\begin{aligned}
& D \rho-\bar{\delta} \kappa=\left(\rho^{2}+|\sigma|^{2}\right)+\rho(\varepsilon+\bar{\varepsilon})-\bar{\kappa} \tau-\kappa(3 \alpha+\bar{\beta}-\pi)+\Phi_{00} \\
& D \sigma-\delta \kappa=\sigma(\rho+\bar{\rho}+3 \varepsilon-\bar{\varepsilon})-\kappa(\tau-\bar{\pi}+\bar{\alpha}+3 \beta)+\Psi_{0} \\
& D \tau-\Delta \kappa=\rho(\tau+\bar{\pi})+\sigma(\bar{\tau}+\pi)+\tau(\varepsilon-\bar{\varepsilon})-\kappa(3 \gamma-\bar{\gamma})+\Psi_{1}+\Phi_{01} \\
& D \alpha-\bar{\delta} \varepsilon=\alpha(\rho+\bar{\varepsilon}-2 \varepsilon)-\beta \bar{\sigma}-\bar{\beta} \varepsilon-\kappa \lambda-\bar{\kappa} \gamma+\pi(\varepsilon+\rho)+\Phi_{10} \\
& D \beta-\delta \varepsilon=\sigma(\alpha+\pi)+\beta(\bar{\rho}-\bar{\varepsilon})-\kappa(\mu+\gamma)-\varepsilon(\bar{\alpha}-\bar{\pi})+\Psi_{1} \\
& D \gamma-\Delta \varepsilon=\alpha(\tau+\bar{\pi})+\beta(\bar{\tau}+\pi)-\gamma(\varepsilon+\bar{\varepsilon})-\varepsilon(\gamma+\bar{\gamma})+\tau \pi-v \kappa+\Psi_{2}+\Phi_{11}-\Lambda \\
& D \lambda-\bar{\delta} \pi=(\rho \lambda+\bar{\sigma} \mu)+\pi(\pi+\alpha-\beta)-v \bar{\kappa}-\lambda(3 \varepsilon-\bar{\varepsilon})+\Phi_{20} \\
& D \mu-\delta \pi=(\bar{\rho} \mu+\sigma \lambda)+\pi(\bar{\pi}-\bar{\alpha}+\beta)-\mu(\varepsilon+\bar{\varepsilon})-v \kappa+\Psi_{2}+2 \Lambda \\
& D v-\Delta \pi=\mu(\pi+\bar{\tau})+\lambda(\bar{\pi}+\tau)+\pi(\gamma-\bar{\gamma})-v(3 \varepsilon-\bar{\varepsilon})+\Psi_{3}+\Phi_{21} \\
& \Delta \lambda-\bar{\delta} v=-\lambda(\mu+\bar{\mu}+3 \gamma-\bar{\gamma})+v(3 \alpha+\bar{\beta}+\pi-\bar{\tau})-\Psi_{4} \\
& \delta \rho-\bar{\delta} \sigma=\rho(\bar{\alpha}+\beta)-\sigma(3 \alpha-\bar{\beta})+\tau(\rho-\bar{\rho})+\kappa(\mu-\bar{\mu})-\Psi_{1}+\Phi_{01} \\
& \delta \alpha-\bar{\delta} \beta=(\mu \rho-\lambda \sigma)+|\alpha|^{2}+|\beta|^{2}-2 \alpha \beta+\gamma(\rho-\bar{\rho})+\varepsilon(\mu-\bar{\mu})-\Psi_{2}+\Phi_{11}+\Lambda \\
& \delta \lambda-\bar{\delta} \mu=v(\rho-\bar{\rho})+\pi(\mu-\bar{\mu})+\mu(\alpha+\bar{\beta})+\lambda(\bar{\alpha}-3 \beta)-\Psi_{3}+\Phi_{21} \\
& \delta v-\Delta \mu=\left(\mu^{2}+|\lambda|^{2}\right)+\mu(\gamma+\bar{\gamma})-\bar{v} \pi+v(\tau-3 \beta-\bar{\alpha})+\Phi_{22} \\
& \delta \gamma-\Delta \beta=\gamma(\tau-\bar{\alpha}-\beta)+\mu \tau-\sigma \nu-\varepsilon \bar{v}-\beta(\gamma-\bar{\gamma}-\mu)+\alpha \bar{\lambda}+\Phi_{12} \\
& \delta \tau-\Delta \sigma=(\mu \sigma+\bar{\lambda} \rho)+\tau(\tau+\beta-\bar{\alpha})-\sigma(3 \gamma-\bar{\gamma})-\kappa \bar{v}+\Phi_{02} \\
& \Delta \rho-\bar{\delta} \tau=(\rho \bar{\mu}+\sigma \lambda)+\tau(\bar{\beta}-\alpha-\bar{\tau})+\rho(\gamma+\bar{\gamma})+\nu \kappa-\Psi_{2}-2 \Lambda \\
& \Delta \alpha-\bar{\delta} \gamma=v(\rho+\varepsilon)-\lambda(\tau+\beta)+\alpha(\bar{\gamma}-\bar{\mu})+\gamma(\bar{\beta}-\bar{\tau})-\Psi_{3} .
\end{aligned}
$$


As identidades de Bianchi da curvatura são normalmente longas e deselegantes assim explicitaremos apenas o caso Ric $=0$ (para o caso geral vide [58]):

$$
\begin{array}{r}
-\bar{\delta} \Psi_{0}+D \Psi_{1}+(4 \alpha+\pi) \Psi_{0}-2(2 \rho+\varepsilon) \Psi_{1}+3 \kappa \Psi_{2}=0 \\
\bar{\delta} \Psi_{1}-D \Psi_{2}-\lambda \Psi_{0}+2(\pi-\alpha) \Psi_{1}+3 \rho \Psi_{2}-2 \kappa \Psi_{3}=0 \\
-\bar{\delta} \Psi_{2}+D \Psi_{3}+2 \lambda \Psi_{1}-3 \pi \Psi_{2}+2(\varepsilon-\rho) \Psi_{3}+\kappa \Psi_{4}=0 \\
\bar{\delta} \Psi_{3}-D \Psi_{4}-3 \lambda \Psi_{2}+2(2 \pi+\alpha) \Psi_{3}-(4 \varepsilon-\rho) \Psi_{4}=0 \\
-\Delta \Psi_{0}+\delta \Psi_{1}+(4 \gamma-\mu) \Psi_{0}-2(2 \tau+\beta) \Psi_{1}+3 \sigma \Psi_{2}=0 \\
-\Delta \Psi_{1}+\delta \Psi_{2}+v \Psi_{0}+2(\gamma-\mu) \Psi_{1}-3 \tau \Psi_{2}+2 \sigma \Psi_{3}=0 \\
-\Delta \Psi_{2}+\delta \Psi_{3}+2 v \Psi_{1}-3 \mu \Psi_{2}+2(\beta-\tau) \Psi_{3}+\sigma \Psi_{4}=0 \\
-\Delta \Psi_{3}+\delta \Psi_{4}+3 v \Psi_{2}-2(\gamma+2 \mu) \Psi_{3}-(\tau-4 \beta) \Psi_{4}=0 .
\end{array}
$$

Fornecemos agora as leis de transformação para os coeficientes de spin e componentes da curvatura sob transformações de Lorentz da base. Considere uma rotação de Classe III, isto é $\mathbf{k} \rightarrow a^{-1} \mathbf{k}, \boldsymbol{\ell} \rightarrow a \boldsymbol{\ell}, \mathbf{m} \rightarrow e^{i \vartheta} \mathbf{m}$ e $\overline{\mathbf{m}} \rightarrow e^{-i \vartheta} \overline{\mathbf{m}}$ onde $a$ e $\vartheta$ são funções reais,

$$
\begin{aligned}
& \kappa \rightarrow \frac{e^{i \vartheta}}{a^{2}} \kappa \quad v \rightarrow a^{2} e^{-i \vartheta} v \quad \varepsilon \rightarrow a^{-1}\left[\varepsilon+\frac{1}{2} D(\ln a+i \vartheta)\right] \\
& \rho \rightarrow \frac{1}{a} \rho \quad \mu \rightarrow a \mu \quad \gamma \rightarrow a\left[\gamma+\frac{1}{2} \Delta(\ln a+i \vartheta)\right] \\
& \sigma \rightarrow \frac{e^{i 2 \vartheta}}{a} \sigma \quad \lambda \rightarrow a e^{-i 2 \vartheta} \lambda \quad \beta \rightarrow e^{i \vartheta}\left[\beta+\frac{1}{2} \delta(\ln a+i \vartheta)\right] \\
& \tau \rightarrow e^{i \vartheta} \tau \quad \pi \rightarrow e^{-i \vartheta} \pi \quad \alpha \rightarrow e^{-i \vartheta}\left[\alpha \frac{1}{2} \bar{\delta}(\ln a+i \vartheta)\right] \\
& \Phi_{00} \rightarrow a^{-2} \Phi_{00} \quad \Phi_{01} \rightarrow a^{-1} e^{i \vartheta} \Phi_{01} \quad \Phi_{02} \rightarrow e^{i 2 \vartheta} \Phi_{02} \\
& \Phi_{22} \rightarrow a^{2} \Phi_{22} \quad \Phi_{12} \rightarrow a e^{-i \vartheta} \Phi_{12} \quad \Phi_{11} \rightarrow \Phi_{11} \\
& \Psi_{0} \rightarrow a^{-2} e^{i 2 \vartheta} \Psi_{0} \quad \Psi_{1} \rightarrow a^{-1} e^{i \vartheta} \Psi_{1} \quad \Psi_{2} \rightarrow \Psi_{2} \\
& \Psi_{3} \rightarrow a e^{-i \vartheta} \Psi_{3} \quad \Psi_{4} \rightarrow a^{2} e^{-i 2 \vartheta} \Psi_{4} .
\end{aligned}
$$

Para rotações de Classe I, isto é $\mathbf{k} \rightarrow \mathbf{k}, \mathbf{m} \rightarrow \mathbf{m}+c \mathbf{k}, \overline{\mathbf{m}} \rightarrow \overline{\mathbf{m}}+\bar{c} \mathbf{k}$ e $\boldsymbol{\ell} \rightarrow \boldsymbol{\ell}+\bar{c} \mathbf{m}+$ $c \overline{\mathbf{m}}+|c|^{2} \mathbf{k}$ onde $c$ é uma função complexa,

$$
\begin{array}{cl}
\kappa \rightarrow \kappa & \tau \rightarrow \tau+c \rho+\bar{c} \sigma+|c|^{2} \kappa \\
\rho \rightarrow \rho+\bar{c} \kappa & \alpha \rightarrow \alpha+\bar{c}(\rho+\varepsilon)+\bar{c}^{2} \kappa \\
\sigma \rightarrow \sigma+c \kappa & \beta \rightarrow \beta+c \varepsilon+\bar{c} \sigma+|c|^{2} \kappa \\
\varepsilon \rightarrow \varepsilon+\bar{c} \kappa & \pi \rightarrow \pi+2 \bar{c} \varepsilon+|c|^{2} \kappa+D \bar{c}
\end{array}
$$




$$
\begin{aligned}
\gamma \rightarrow \gamma+c \alpha+\bar{c}(\beta+\tau)+|c|^{2}(\rho+\varepsilon)+\bar{c}^{2} \sigma+\bar{c}^{2} \kappa \\
\lambda \rightarrow \lambda+\bar{c}(2 \alpha+\pi)+\bar{c}^{2}(\rho+2 \varepsilon)+\bar{c}^{3} \kappa+\bar{\delta} \bar{c}+\bar{c} D \bar{c} \\
\mu \rightarrow \mu+c \pi+2 \bar{c} \beta+2|c|^{2} \varepsilon+\bar{c}^{2} \sigma+c \bar{c}^{2} \kappa+\delta \bar{c}+c D \bar{c} \\
\nu \rightarrow v+c \lambda+\bar{c}(\mu+2 \gamma)+\bar{c}^{2}(\tau+2 \beta)+\bar{c}^{3} \sigma+|c|^{2}(\pi+2 \alpha)+ \\
\quad+c \bar{c}^{2}(\rho+2 \varepsilon)+\bar{c}^{3} \kappa+\left(\Delta+\bar{c} \delta+c \bar{\delta}+|c|^{2} D\right) \bar{c}
\end{aligned}
$$

$$
\begin{aligned}
& \Phi_{00} \rightarrow \Phi_{00} \\
& \Phi_{01} \rightarrow \Phi_{01}+c \Phi_{00} \\
& \Phi_{02} \rightarrow \Phi_{02}+2 c \Phi_{01}+c^{2} \Phi_{00} \\
& \Phi_{22} \rightarrow \Phi_{22}+2 \bar{c} \Phi_{12}+2 c \Phi_{21}+\bar{c}^{2} \Phi_{02}+4|c|^{2} \Phi_{11}+c^{2} \Phi_{20}+2 \bar{c} \bar{c}^{2} \Phi_{01}+2 \bar{c} c^{2} \Phi_{10}+|c|^{4} \Phi_{00} \\
& \Phi_{12} \rightarrow \Phi_{12}+\bar{c} \Phi_{02}+2 c \Phi_{11}+2|c|^{2} \Phi_{01}+c^{2} \Phi_{10}+\bar{c} c^{2} \Phi_{00} \\
& \Phi_{11} \rightarrow \Phi_{11}+\bar{c} \Phi_{01}+c \Phi_{10}+|c|^{2} \Phi_{00} \\
& \Psi_{0} \rightarrow \Psi_{0} \\
& \Psi_{1} \rightarrow \Psi_{1}+\bar{c} \Psi_{0} \\
& \Psi_{2} \rightarrow \Psi_{2}+2 \bar{c} \Psi_{1}+\bar{c}^{2} \Psi_{0} \\
& \Psi_{3} \rightarrow \Psi_{3}+3 \bar{c} \Psi_{2}+3 \bar{c}^{2} \Psi_{1}+\bar{c}^{3} \Psi_{0} \\
& \Psi_{4} \rightarrow \Psi_{4}+4 \bar{c} \Psi_{3}+6 \bar{c}^{2} \Psi_{2}+4 \bar{c}^{3} \Psi_{1}+\bar{c}^{4} \Psi_{0} .
\end{aligned}
$$

Para as transformações de Classe II fazemos a reflexão $\mathbf{k} \leftrightarrow \boldsymbol{\ell}$ e $\mathbf{m} \leftrightarrow \overline{\mathbf{m}}$ e as respectivas mudanças nos coeficientes de spin e procedemos analogamente às transformações de Classe I. 


\title{
Apêndice B
}

\section{Tópicos de Estrutura Global}

\begin{abstract}
A compreensão do que consiste um buraco negro, bem como de sua dinâmica 1 depende enormemente de um conhecimento detalhado da estrutura causal e assintótica de um espaço-tempo. Reunimos aqui as definições e proposições essenciais para a conveniência do leitor, deferindo todas as demonstrações às referências, por exemplo [4] ou [59].
\end{abstract}

\section{B.1 Causalidade}

Definição B.1.1 Seja $(\mathcal{M}, \mathbf{g})$ um espaço-tempo e $p \in \mathcal{M}$. Considere os vetores $\mathbf{t}$ de $T_{p}$ tais que $(\mathbf{t} \mid \mathbf{t})<0$, isto é os vetores tipo-tempo. Definamos uma relação de equivalência entre vetores do espaço tangente a p por $\mathbf{t} \equiv \mathbf{u}$ se, e somente se, $(\mathbf{t} \mid \mathbf{u})<0$. Essa relação de equivalência divide os vetores tipo-tempo de $T_{p}$ em duas partições. Escolhemos uma delas arbitrariamente e a denominamos conjunto orientado a futuro e a outra conjunto orientado ao passado.

Definição B.1.2 Seja $(\mathcal{M}, \mathbf{g})$ um espaço-tempo e $\gamma$ uma curva fechada arbitrária tal que $\gamma(0)=$ $\gamma(1)=p$. Escolha um vetor de $T_{p}$ orientado ao futuro e faça o transporte paralelo ao longo de $\gamma$ de $\gamma(0)$ a $\gamma(1)$. Se o vetor transportado paralelamente for orientado ao futuro então $(\mathcal{M}, \mathbf{g})$ é orientável no tempo e do contrário não-orientável no tempo. 
Proposição B.1.3 Seja $(\mathcal{M}, \mathbf{g})$ uma espaço-tempo orientável no tempo. Então existe um campo vetorial tipo-tempo suave em $\mathcal{M}$. Conversamente se existe tal campo vetorial então o espaçotempo é orientável no tempo.

Definição B.1.4 Seja $(\mathcal{M}, \mathbf{g})$ um espaço-tempo e $U$ um aberto em $\mathcal{M}$ tal que para quaisquer $p, q \in U$ existe uma geodésica única que passa por p e q e cujos pontos intermediários estão todos contidos no aberto. Então U é uma vizinhança normal convexa.

Definição B.1.5 Seja $(\mathcal{M}, \mathbf{g})$ um espaço-tempo e $p \in \mathcal{M}$. Então existe uma vizinhança normal convexa $U$ de $p$ onde sendo $\lambda\left(t_{1}\right), \lambda\left(t_{2}\right) \in U, t_{1}<t_{2}$, existe uma curva diferenciável, não-única, $\lambda$ de $\lambda\left(t_{1}\right)$ a $\lambda\left(t_{2}\right)$ com vetores tangentes tipo-tempo orientados para o futuro. Essa curva $\lambda e ́$ dita curva tipo-tempo dirigida ao futuro. Analogamente caso os vetores tangentes sejam tipo-tempo orientados ao futuro ou vetores nulos então $\lambda$ é uma curva causal dirigida ao futuro.

Definição B.1.6 Seja $(\mathcal{M}, \mathbf{g})$ um espaço-tempo e $p \in \mathcal{M}$. Considere o conjunto $I^{+}(p):=\{q \in$ $\mathcal{M} \mid \exists \gamma$ tipo-tempo dirigida ao futuro tal que $\gamma(0)=p$ e $\gamma(1)=q$. Chamamos $I^{+}(p)$ o futuro cronológico de $p$. Analogamente temos $I^{-}(p)$ o passado cronológico de $p$, e $J^{ \pm}(p)$ o futuro (passado) causal de $p$. Note que em particular $p \in J^{+}(p)$. Seja $S \subset \mathcal{M}$ então definimos o seu futuro cronológico por $I^{+}(S) \equiv \bigcup_{p \in S} I^{+}(p)$.

Teorema B.1.7 Seja $(\mathcal{M}, \mathbf{g})$ um espaço-tempo e $p \in \mathcal{M}$. Então existe uma vizinhança normal convexa $U$ tal que $p \in U$. Denotanto $\left.I^{+}(p)\right|_{u}$ pela intersecção do futuro cronológico de $p$ com a vizinhança normal temos que $\left.\dot{I}^{+}(p)\right|_{u}$ é gerado pelas geodésicas nulas dirigidas ao futuro com origem em $p$.

Definição B.1.8 Seja um subconjutno $S \subset \mathcal{M}$ tal que $I^{+}(S) \cap S=\emptyset$. Então $S$ é um conjunto acronal.

Definição B.1.9 Seja $\gamma(t)$ uma curva causal dirigida ao futuro. Considere $p \in \mathcal{M}$ tal que $\forall O \ni p \exists t_{0}(O)$ de modo que se $t>t_{0} \Rightarrow \gamma(t) \in O$. Então $p$ é um ponto final futuro de $\gamma$. Uma curva sem pontos finais futuros é inextensível ao futuro. Analogamente trocando futuro por passado podemos definir uma curva inextensível ao passado. 
Definição B.1.10 Seja $\gamma$ uma geodésica a partir de $p \in$ Minextensível em uma dada orientação. Se na direção de tal orientação a geodésica cobre uma variação finita de parâmetro afim, então a geodésica é dita incompleta. Um espaço-tempo é geodesicamente completo se, e somente se, todas as geodésicas nele são completas.

Definição B.1.11 Seja $(\mathcal{M}, \mathbf{g})$ um espaço-tempo e $p \in \mathcal{M}$. Se para qualquer vizinhança $O$ de p existe vizinhança $U \subset O, p \in U$ tal que nenhuma curva causal intercepta $U$ mais de uma vez então $(\mathcal{M}, \mathbf{g})$ é um espaço-tempo fortemente causal.

Proposição B.1.12 Seja $\mathbf{g}$ uma métrica e um vetor tipo-tempo $\mathbf{t}$ de covetor $\vartheta$, então a métrica $\tilde{\mathbf{g}}=\mathbf{g}-\vartheta \otimes \vartheta$ é de assinatura lorentziana e todo vetor causal de $\mathbf{g}$ é tipo-tempo em $\tilde{\mathbf{g}}$.

Definição B.1.13 Seja um espaço-tempo $(\mathcal{M}, \mathbf{g})$ tal que existe um campo vetorial tipo-tempo contínuo, para o qual o espaço-tempo $(\mathcal{M}, \tilde{\mathbf{g}})$ não possui curvas tipo-tempo fechadas, então $(\mathcal{M}, \mathrm{g})$ é estavelmente causal.

Proposição B.1.14 Um espaço-tempo estavelmente causal é fortemente causal.

Definição B.1.15 Seja $S \subset \mathcal{M}$ um conjunto fechado e acronal. O conjunto $D^{+}(S):=\{p \in$ $\mathcal{M} \mid \forall \lambda$ causal inextensível ao passado com $p \in \lambda$ intercepta $S\}$ é o domínio de dependência futuro de $S$. O domínio de dependência de $S$ é $D(S)=D^{+}(S) \cup D^{-}(S)$.

Definição B.1.16 Seja $\Sigma \subset \mathcal{M}$ um conjunto fechado acronal tal que $D(\Sigma)=\mathcal{M}$, então $\Sigma e ́$ uma superfície de Cauchy de $\mathcal{M}$. Se $(\mathcal{M}, \mathbf{g})$ possui uma superfície de Cauchy então é um espaço-tempo globalmente hiperbólico.

Lema B.1.17 Todo espaço-tempo globalmente hiperbólico é estavelmente causal.

\section{B.2 Asymtopia}

Definição B.2.1 Seja $(\mathcal{M}, \mathbf{g})$ um espaço-tempo tal que exista um espaço-tempo $(\widetilde{\mathcal{M}}, \tilde{\mathbf{g}})$ e um homeomorfismo $\theta: \mathcal{M} \rightarrow \widetilde{\mathcal{M}}$ que embute $\mathcal{M}$ em $\widetilde{\mathcal{M}}$ e 
1. Mé subvariedade de $\widetilde{\mathcal{M}}$ com fronteira suave $\partial \mathcal{M}$;

2. Existe uma função suave $\Omega$ em $\widetilde{\mathcal{M}}$ tal que $\tilde{\mathbf{g}}=\Omega^{2} \mathbf{g}$ (são métricas conformes);

3. Em $\partial \mathcal{M}$ temos $\Omega=0 e \nabla \Omega \neq 0$;

4. Toda geodésica nula tem dois pontos finais em $\partial \Omega$.

Então o espaço-tempo $(\mathcal{M}, \mathbf{g})$ é assintoticamente simples.

Proposição B.2.2 Um espaço-tempo assintoticamente simples é globalmente hiperbólico.

Proposição B.2.3 dMé uma superfície nula.

Definição B.2.4 Seja $\mathscr{I}^{+}$o conjunto de pontos de $\partial \mathscr{M}$ tal que $\mathcal{M}$ está no passado de $\mathscr{I}^{+}$, isto é $\mathscr{I}^{+}$é o conjunto de pontos finais futuros das geodésica nulas de $\mathcal{M}$, então $\mathscr{I}^{+}$é o infinito futuro nulo de $\mathcal{M}$. Analogamente definimos $\mathscr{I}^{-}$o infinito passado nulo.

Definição B.2.5 Seja $(\mathcal{M}, \mathbf{g})$ um espaço-tempo assintoticamente simples para o qual o espaçotempo $(\widetilde{\mathcal{M}}, \tilde{\mathbf{g}})$ é tal que uma vizinhança $\tilde{U}$ de $\partial \mathcal{M}$ tem intersecção $\tilde{U} \cap \mathcal{M}$ isométrica a um aberto U de $\mathcal{M}$. Então $(\mathcal{M}, \mathrm{g})$ é fracamente assintoticamente simples.

Definição B.2.6 Seja $(\mathcal{M}, \mathbf{g})$ um espaço-tempo fracamente assintoticamente simples. Se existe $\tilde{V} \subset \widetilde{\mathcal{M}} \operatorname{com} \overline{\mathcal{M} \cap J^{-}\left(\mathscr{I}^{+}\right)} \subset \tilde{V}$ tal que $(\tilde{V}, \tilde{\mathbf{g}})$ é globalmente hiperbólico, então $(\mathcal{M}, \mathbf{g})$ é fortemente assintoticamente previsível. 


\section{Apêndice C}

\section{Congruências Geodésicas}

$\mathrm{H}$

ISTORICAMENTE o resultado que mostrou o poder dos métodos de geometria diferencial na Relatividade Geral foi o primeiro teorema de singularidade de Penrose [60]. A partir deste a compreensão dos buracos negros, tanto no âmbito de sua formação quanto de sua evolução, adquiriu um caráter geral não mais necessitando de soluções específicas. Vamos aqui fazer um breve resumo do tratamento de congruências de geodésicas nulas e das condições que implicam na formação de singularidades. Esses resultados, junto com a conjectura de censura cósmica, implicam na inevitabilidade da formação de buracos negros em certas condições. As demonstrações aqui não fornecidas podem ser todas encontradas em [17] ou [4].

\section{C.1 Campos de Jacobi}

Definição C.1.1 Considere uma variedade $\mathcal{M}$ e um aberto $O \subset \mathcal{M}$. Seja $\left\{\lambda_{n}\right\}$ uma família de curvas tais que para qualquer $p \in O$ existe apenas uma curva $\lambda_{m}$ tal que $p \in \lambda_{m}$. A família $\left\{\lambda_{n}\right\}$ é uma congruência em $O$.

Vamos nos focar nas congruências de geodésicas nulas conforme discutido na seção 2.1. Seja $\mathcal{B}=[\mathfrak{B}]$ a matriz do operador forma [2.1.2] em uma dada base. Nesta base 
podemos escrever a matriz usando os escalares ópticos:

$$
\mathcal{B}=-\frac{1}{2}\left[\begin{array}{cc}
(\rho+\bar{\rho})+(\sigma+\bar{\sigma}) & i(\rho-\bar{\rho})+i(\sigma-\bar{\sigma}) \\
-i(\rho-\bar{\rho})+i(\sigma-\bar{\sigma}) & (\rho+\bar{\rho})-(\sigma+\bar{\sigma})
\end{array}\right] .
$$

Denotando $D \equiv \nabla_{\mathbf{k}}$ podemos obter $D \mathcal{B}$ das equações de Sachs e dos seus complexos conjugados, de onde obtemos

$$
\begin{aligned}
D \mathcal{B}= & -\frac{1}{2}\left[\begin{array}{cc}
\left(\rho^{2}+\bar{\rho}^{2}\right)+2|\sigma|^{2}+(\rho+\bar{\rho})(\sigma+\bar{\sigma}) & i\left[\left(\rho^{2}-\bar{\rho}^{2}\right)+(\rho+\bar{\rho})(\sigma-\bar{\sigma})\right] \\
-i\left[\left(\rho^{2}-\bar{\rho}^{2}\right)-(\rho+\bar{\rho})(\sigma-\bar{\sigma})\right] & \left(\rho^{2}+\bar{\rho}^{2}\right)+2|\sigma|^{2}-(\rho+\bar{\rho})(\sigma+\bar{\sigma})
\end{array}\right] \\
& +\frac{1}{2}\left[\begin{array}{cc}
\left(\operatorname{Ric}(\mathbf{k}, \mathbf{k})+\mathrm{C}_{\mathbf{k x k x}}-\mathrm{C}_{\mathbf{k y k y}}\right) & -\left(\mathrm{C}_{\mathbf{k x k y}}+\mathrm{C}_{\mathbf{k y k x}}\right) \\
\left(\mathrm{C}_{\mathbf{k x k y}}+\mathrm{C}_{\mathbf{k y k x}}\right) & \operatorname{Ric}(\mathbf{k}, \mathbf{k})-\mathrm{C}_{\mathbf{k x k x}}+\mathrm{C}_{\mathbf{k y k y}}
\end{array}\right]
\end{aligned}
$$

É simples de ver que a primeira matriz é o quadrado de $\mathcal{B}$ de modo que obtemos

$$
D \mathcal{B}+\mathcal{B}^{2}+\mathcal{R}=0
$$

onde $\mathcal{R}$ é uma matriz $2 \times 2$ tal que $\mathcal{R}=\mathcal{R}^{\top}$, trace $\mathcal{R}=-\operatorname{Ric}(\mathbf{k}, \mathbf{k}),-2 \mathcal{R}_{11}=\operatorname{Ric}(\mathbf{k}, \mathbf{k})+$ $\mathrm{C}_{\text {kxkx }}-\mathrm{C}_{\text {kyky }}$ e $\mathcal{R}_{12}=\mathrm{C}_{\text {kxky }}$.

Lema C.1.2 Seja k o campo tangente a uma congruência geodésica nula ortogonal a hiperfícies. Suponha $\operatorname{Ric}(\mathbf{k}, \mathbf{k}) \geq 0$ (condição de energia forte). Então se a exansão assume um valor $\theta_{r}<0$ em $\lambda=$ r então $\theta \rightarrow-\infty$ em um parâmetro afim $\lambda \leq r+2 /\left|\theta_{r}\right|$, desde que a geodésica se estenda até tal ponto.

Demonstração Se a congruência é ortogonal a hiperfícies temos $\omega=0$. Tomando o traço de C.1.3 temos a primeira equação de Sachs $D \theta=-\frac{1}{2} \theta^{2}-2|\sigma|^{2}-\operatorname{Ric}(\mathbf{k}, \mathbf{k})$ que com a condição de energia forte implica $D \theta+\frac{1}{2} \theta^{2} \leq 0$ e portanto $-\frac{1}{\theta^{2}} D \theta \geq \frac{1}{2}$. Disso tiramos que $D\left(\theta^{-1}\right) \geq \frac{1}{2}$ o que implica $\theta^{-1} \geq \theta_{r}^{-1}+\frac{1}{2} \lambda$. Se $\theta_{r}<0$ como no enunciado então para $\lambda \rightarrow \frac{2}{\left|\theta_{r}\right|}$ temos que $\theta^{-1} \rightarrow 0$ e portanto $\theta \rightarrow-\infty$.

Definição C.1.3 Seja $\gamma$ uma geodésica de tangente $\mathbf{v}$ e $D \equiv \nabla_{\mathbf{v}}$ então um campo vetorial $\mathbf{Z}$ que satisfaça $D^{2} \mathbf{Z}=R_{\mathbf{Z v}}(\mathbf{v})$ é um campo de Jacobi em $\gamma$.

Definição C.1.4 Seja $p, q \in \gamma$ onde $\gamma$ é uma geodésica. Se existe um campo de Jacobi não indenticamente nulo mas que se anula em p e q, então tais pontos são ditos conjugados. 
Proposição C.1.5 Seja $\mu$ uma geodésica nula de campo tangente $\mathbf{k}$ e p,q $q \mu$. Considere agora

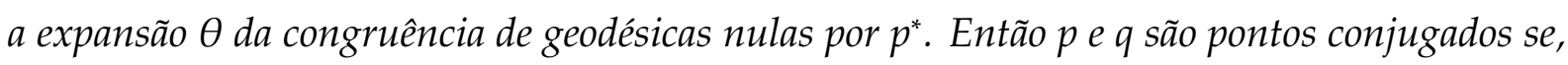
e somente se, $\theta \rightarrow-\infty$ em $q$.

Demonstração Considere um vetor de desvio geodésico $\mathbf{Z} \in \mathbf{k}^{\perp} / \mathbf{k}$ nulo em $p$, então ele é um campo de Jacobi [4], portanto satisfaz a equação

$$
\frac{d^{2}}{d \lambda^{2}} Z^{\alpha}=\sum_{\beta=1}^{2} R_{\beta \mathbf{k}}(\mathbf{k})^{\alpha} Z^{\beta}
$$

onde $R_{\beta \mathbf{k}}(\mathbf{k})^{\alpha}$ é a matriz correspondente ao tensor $R_{\cdot \mathbf{k}}(\mathbf{k})$ em uma base arbitrária do espaço $\mathbf{k}^{\perp} / \mathbf{k}$ e $\lambda$ é o parâmetro afim de $\mathbf{k}$. $Z^{\alpha}$ tem como solução geral

$$
Z^{\alpha}(\lambda)=\sum_{\beta=1}^{2} A_{\beta}^{\alpha}(\lambda) \frac{d Z^{\beta}}{d \lambda}(0)+\sum_{\beta=1}^{2} A_{\beta}^{\prime \alpha} Z^{\beta}(0),
$$

onde $\mu(0)=p$. Se as geodésicas emanam de $p$ então $Z^{\beta}(0)=0$ e substituindo (C.1.5) em C.1.4 obtemos

$$
\frac{d^{2}}{d \lambda^{2}} A_{\beta}^{\alpha}=\sum_{\gamma=1}^{2} R_{\gamma \mathbf{k}}(\mathbf{k})^{\alpha} A_{\beta}^{\gamma} .
$$

Temos então $A^{\alpha}{ }_{\beta}(0)=0$ e $D A^{\alpha}{ }_{\beta}(0)=\delta_{\beta}^{\alpha}$. Se $q$ é conjugado a $p$ então $\operatorname{det} A \neq 0$ entre $p$ e $q$ e $\operatorname{det} A=0$ em $p$ e $q$. Agora tomemos a derivada em relação a $\lambda$ do vetor de desvio geodésico $D Z^{\mu}=\nabla_{\mathbf{k}}\left(\mathbf{E}^{\mu} \mid \mathbf{Z}\right)=\left(\mathbf{E}^{\mu} \mid \nabla_{\mathbf{k}} \mathbf{Z}\right)$. Para avaliar esse termo utilizamos a derivada de Lie do vetor de desvio $£_{\mathbf{k}} \mathbf{Z}=[\mathbf{Z}, \mathbf{k}]=0 \Rightarrow \nabla_{\mathbf{k}} \mathbf{Z}=\nabla_{\mathbf{Z}} \mathbf{k}$, e portanto

$$
\frac{d}{d \lambda} Z^{\mu}=\left(\mathbf{E}^{\mu} \mid \nabla_{\mathbf{Z}} \mathbf{k}\right)=\sum_{v} \mathfrak{B}^{\mu}{ }_{v} Z^{v}
$$

e agora substituindo (C.1.5) em (C.1.7)

$$
\sum_{v} \frac{d A^{\mu}{ }_{v}(\lambda)}{d \lambda} \frac{d Z^{v}(0)}{d \lambda}=\sum_{v, \rho} \mathfrak{B}^{\mu}{ }_{\rho} A^{\rho}{ }_{v}(\lambda) \frac{d Z^{v}(0)}{d \lambda}
$$

o que implica $D A=\mathfrak{B} A$ ou $\mathfrak{B}=(D A) A^{-1}$. Tomando o traço da última expressão, e lembrando a definição da expansão [2.1.3], $\theta=\operatorname{trace}\left[(D A) A^{-1}\right]=(\operatorname{det} A)^{-1} D(\operatorname{det} A)[4]$. Portanto $\theta=D \ln |\operatorname{det} A|$ e $\theta \rightarrow-\infty \Leftrightarrow \operatorname{det} A \rightarrow 0$.

*Note que se uma família de geodésicas possui um ponto em comum então nesse ponto a congruência é singular, isto é não está bem definida. Perceba que não supomos nada a respeito da congruência em $p$. 


\section{C.2 Formação de Singularidades}

Tendo os resultados elementares sobre pontos conjugados e sua relação com incompleteza das geodésicas podemos agora formular o problema da formação de singularidades.

Definição C.2.1 Seja $\Sigma$ uma hiperfície tipo-espaço de $\mathcal{M}$ e $p$ um ponto em uma geodésica que faz parte da congruência geodésica ortogonal à hiperfície, então pé conjugado a $\Sigma$ se, e somente se, existe um campo desvio geodésico não nulo na hiperfície e nulo em $p$.

Em analogia ao lema C.1.2 se existir uma superfície suave tipo-espaço bidimensional com uma congruência geodésica nula ortogonal tal que a expansão seja negativa na superfície, então existe um ponto conjugado a ela em uma distância finita do parâmetro afim ao longo de uma geodésica nula. O resultado mais importante para superfícies se refere àquelas que são compactas.

Teorema C.2.2 Seja $(\mathcal{M}, \mathbf{g})$ um espaço-tempo globalmente hiperbólico e K uma subvariedade bidimensional tipo espaço compacta e orientável. Então todo $p \in \dot{J}^{+}(K)$ pertence a uma geodésica nula dirigida ao futuro começando em K e ortogonal a $\mathrm{K}$ que não possui ponto conjugado a $\mathrm{K}$ entre p e $K$.

Também relevantes serão as relações entre a estrutura causal das geodésicas e os pontos cojugados.

Teorema C.2.3 Seja u uma curva causal suave e $p, q \in \mu$. Então não existe uma família uniparamétrica de curvas causais $\lambda_{\alpha}$ conectando $p$ a q, tal que $\lambda_{0}=\mu$ e $\lambda_{\alpha}$ é tipo-tempo para $\forall \alpha>0$, se, e somente se, $\mu$ é uma geodésica nula sem pontos conjugados a $p$ entre este ponto e $q$.

Teorema C.2.4 Seja $(\mathcal{M}, \mathbf{g})$ um espaço-tempo fortemente causal. Sejam $p, q \in \mathcal{M}$ e $q \in J^{+}(p)$ e considere a função comprimento definida no espaço de todas as curvas causais contínuas dirigidas ao futuro que conectam $p$ a q. Então a função comprimento terá um máximo para a geodésica sem pontos conjugados a p entre o mesmo e q. 
Os últimos teoremas tem generalizações naturais considerando a relação de conjugação entre um ponto $p$ e uma hiperfície tipo-espaço, acronal no caso de C.2.4 para definirmos o espaço de curvas causais dirigidas ao futuro correspondente. O ponto central dos teoremas de singularidade para buracos negros revolve em torno de uma superfície espacial que certamente possui pontos conjugados.

Definição C.2.5 Seja uma superfície tipo-espaço bidimensional compacta $\mathscr{T}$ tal que as duas congruências geodésicas nulas ortogonais a $\mathscr{T}$ tem expansão negativa em todos os pontos da superfície. Então $\mathscr{T}$ é uma superfície aprisionada fechada.

Agora veremos que a existência de uma superfície aprisionada fechada é incompatível com a completeza geodésica em certas condições [60]

Teorema C.2.6 (Penrose) Um espaço-tempo $(\mathcal{M}, \mathbf{g})$ não pode ser geodesicamente nulo completo se satisfizer todas as seguintes condições:

1. Existir uma superfície aprisionada fechada $\mathscr{T}$ em $\mathcal{M}$;

2. $\operatorname{Ric}(\mathbf{k}, \mathbf{k}) \geq 0$ para qualquer vetor nulo $\mathbf{k}$;

3. Existir uma superfície de Cauchy não-compacta $\mathscr{H} \in \mathcal{M}$.

Demonstração Se existe uma superfície de Cauchy então o espaço-tempo é globalmente hiperbólico. Então $\dot{J}^{+}(\mathscr{T})$ é gerado por geodésicas nulas com ponto finito passado em $\mathscr{T}$ C.2.2. Das duas primeiras condições do enunciado e de C.2.3 temos então que todas as geodésicas nulas que geram a fronteira do futuro causal de $\mathscr{T}$ contém um ponto conjugado à superfície aprisionada. Assim os pontos dessas geodésicas além do ponto conjugado pertencem a $I^{+}(\mathscr{T})\left[\right.$ C.2.4]. Portanto os geradores de $\dot{J}^{+}(\mathscr{T})$ possuem ponto final futuro antes de ou no ponto conjugado à superfície aprisionada. Sendo a superfície compacta e orientável vamos escolher uma tetrada nula complexa continuamente nela. Vamos denotar $\vartheta=\max \{\theta,-\mu\}$ na superfície, ou seja o máximo das expansões de ambas as congruências nulas, e $f_{+}: \mathscr{T} \times[0,|\vartheta| / 2] \rightarrow \mathcal{M}$ pela função $f_{+}(q, z)$ que corresponde ao ponto que está a $z$ parâmetro afim ao longo da geodésica nula gerada 
por $\mathbf{k}$ com origem em $q$. Analogamente $f_{-}$trocando $\mathbf{k}$ por $\boldsymbol{\ell}$. Dado que o domínio dessas funções é compacto e as funções contínuas então as imagens $f_{ \pm}(\mathscr{T} \times[0,|\vartheta| / 2])$ também serão compactas bem como suas uniões $\mathscr{A}=f_{+}(\mathscr{T} \times[0,|\vartheta| / 2]) \cup f_{-}(\mathscr{T} \times[0,|\vartheta| / 2])$. Porém $\dot{J}^{+}(\mathscr{T}) \subset \mathscr{A}$ o que implica que tal conjunto também é compacto pois é fechado. Note que a compacidade de $\dot{J}^{+}(\mathscr{T})$ depende do espaço-tempo ser geodesicamente completo, caso contrário existiria um ponto no conjunto que não poderia ser aproximado arbitrariamente por pontos pertencentes às geodésicas que geram o conjunto. Agora pela terceira propriedade do enunciado existe um campo vetorial $C^{1}$ que intercepta $\mathscr{H}$ uma única vez. Além disso esse campo intercepta $\dot{J}^{+}(\mathscr{T})$ uma única vez pois tal conjunto é acronal. Assim sendo existe um mapa contínuo $\Psi: \dot{J}^{+}(\mathscr{T}) \rightarrow \mathscr{H}$. Se $\dot{J}^{+}(\mathscr{T})$ é compacto então sua imagem do mapa $\Psi$ também o é e portanto $\Psi\left(\dot{J}^{+}(\mathscr{T})\right) \subset \mathscr{H}$. Assim sendo a imagem de $\Psi$ contém uma fronteira em $\mathscr{H}$. No entanto, de um resultado geral para subconjuntos de $\mathcal{M}[17]$, temos que $\dot{J}^{+}(\mathscr{T})$ é uma hiperfície tridimensional acronal, e portanto sem fronteira. Portanto $\dot{J}^{+}(\mathscr{T})$ não pode ser um conjunto compacto e o espaço-tempo não é geodesicamente completo. 